University of Louisville

ThinkIR: The University of Louisville's Institutional Repository

Electronic Theses and Dissertations

$12-2020$

\title{
The green house nursing home model: the GH elements and their impact on quality of care in nursing homes.
}

Deborah Kaminka Niyongabo

University of Louisville

Follow this and additional works at: https://ir.library.louisville.edu/etd

Part of the Health Services Research Commons

\section{Recommended Citation}

Niyongabo, Deborah Kaminka, "The green house nursing home model: the GH elements and their impact on quality of care in nursing homes." (2020). Electronic Theses and Dissertations. Paper 3540.

https://doi.org/10.18297/etd/3540

This Doctoral Dissertation is brought to you for free and open access by ThinkIR: The University of Louisville's Institutional Repository. It has been accepted for inclusion in Electronic Theses and Dissertations by an authorized administrator of ThinkIR: The University of Louisville's Institutional Repository. This title appears here courtesy of the author, who has retained all other copyrights. For more information, please contact thinkir@louisville.edu. 


\title{
THE GREEN HOUSE NURSING HOME MODEL: THE GH ELEMENTS AND THEIR IMPACT ON QUALITY OF CARE IN NURSING HOMES
}

\author{
By \\ Deborah Kaminka Niyongabo \\ A.A., Montgomery College, 2010 \\ B.S., University of Maryland, 2012 \\ M.P.H., Liberty University, 2015
}

\begin{abstract}
A Dissertation
Submitted to the Faculty of the School of Public Health and Information Sciences of the University of Louisville in Partial Fulfillment of the Requirements for the Degree of

Doctor of Philosophy in Public Health Science

Department of Health Management and Systems Sciences

School of Public Health

University of Louisville

Louisville, Kentucky
\end{abstract}

December 2020 

THE GREEN HOUSE NURSING HOME MODEL: THE GH ELEMENTS AND

\section{THEIR IMPACT ON QUALITY OF CARE IN NURSING HOMES}

By

Deborah Kaminka Niyongabo

A.A., Montgomery College, 2010

B.S., University of Maryland, 2012

M.P.H., Liberty University, 2015

A Dissertation Approved on

November 13, 2020

by the following Dissertation Committee:

Dissertation Committee Chair: Christopher E. Johnson, Ph.D.

Dissertation Committee Member: J'Aime C. Jennings, Ph.D.

Dissertation Committee Member: Liza M. Creel, Ph.D.

Dissertation Committee Member: David J. Roelfs, Ph.D. 


\section{DEDICATION}

I dedicate this dissertation to my Lord and Savior, Jesus-Christ; to my parents Celestin Niyongabo and Seraphine Manirambona; to my sisters Muriel Niyongabo, Aurelie Niyongabo, and Martine Niyongabo. To my extended family members around the world. To my friends, Dr. Baraka Muvuka, Kelsey White, Elizabeth Barnes, and Honey Mary. I also dedicate this dissertation to Dr. Wanjiru Kamau. 


\section{ACKNOWLEDGMENTS}

I would like to thank my dissertation chair, Dr. Johnson who has been present since the first day of my program. He guided me with his wisdom during my dissertation. In his classes, he enhanced my curiosity for organizations and the way they operate within the field of health services research. I also would like to acknowledge Dr. Creel who worked beyond and above her duties to support me in every step of the way. She was not only a Professor or a dissertation committee member, but also a remarkable mentor. I would like to thank Dr. Roelfs who guided me with patience and understanding by providing important advice during the dissertation process. Dr. Jennings helped me at crucial times of the process. She provided me with advice that I incorporated in my project. My acknowledgment also goes to thank Darla Samuelsen who from the first day of entering the $\mathrm{PhD}$ program has helped. Last but not least, I would like to thank my parents who were my first cheerleaders especially during difficult times. I would also like to thank my sisters, other family members, and friends who encouraged me through prayers along the journey. 


\section{ABSTRACT \\ THE GREEN HOUSE NURSING HOME MODEL: THE GH ELEMENTS AND THEIR IMPACT ON QUALITY OF CARE IN NURSING HOMES \\ Deborah K. Niyongabo}

November 13, 2020

Nursing homes (NHs) have been described as hospital-like facilities where residents are more likely to experience isolation or abuse. The Green House (GH) model is a type of culture change which focuses on deinstitutionalizing traditional NHs to provide better quality of care and quality of life to NH residents. The two main objectives of this study are: to describe the GH model and its elements and to identify which GH elements impact the quality of care. This study also seeks to identify organizational theories used in the GH analysis. The next objective is to analyze the impact of the GH model elements on the deficiency citations. Lastly, the other objective is to examine the impact of the GH model elements on the indicators of quality of care.

For the methods section, the Preferred Reporting Items for Systematic Reviews and Meta-Analyses (PRISMA) and five databases were used. For the quantitative section, two methodological strategies were used to examine the data with Stata 14 including a propensity score and a difference-in-difference. Eighteen articles analyzed the GH model and no organizational theories were identified. Staff and physical structure were the most assessed GH elements in the literature. The GH model had a positive effect on preventable hospitalizations, falls, and in residents with pressure ulcers and catheters. 
For the quantitative section of the study, 5,040 facility-year observations were included in the study sample with $26 \mathrm{GH}$ facilities and 254 facilities for the years 2000 to 2017. Even though the results were not statistically significant, the incidence rate ratio for deficiency citations was lower in $\mathrm{GH}$ facilities than in non-GH facilities. For the last objective, the incidence rate ratio for bowel incontinence was lower in GH facilities than in non-GH facilities (IRR: 0.920; P-value: 0.002 ), but no statistically significant difference was found between the impact of the GH model and falls and bladder incontinence. Our findings showed that there was an association between the GH model and the prevalence of bowel incontinence, but not with the other indicators of quality of care. More research needs to be done to contribute to the long-term care field. 


\section{TABLE OF CONTENTS}

PAGE

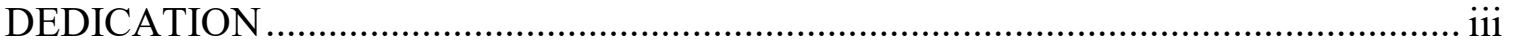

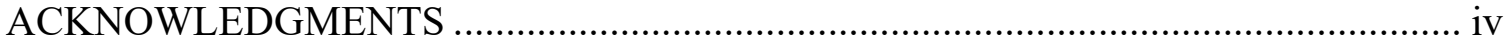

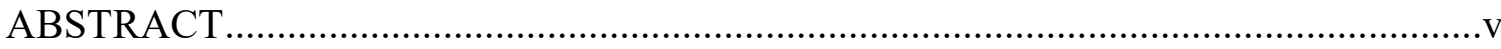

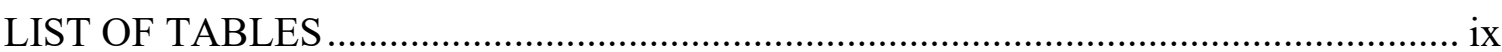

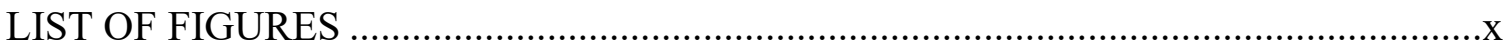

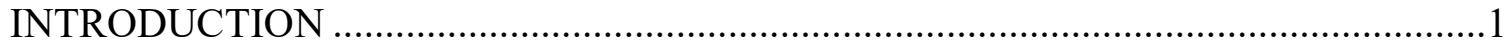

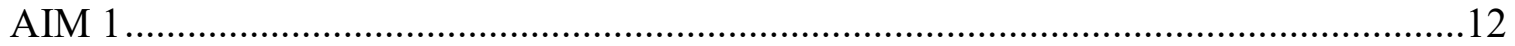

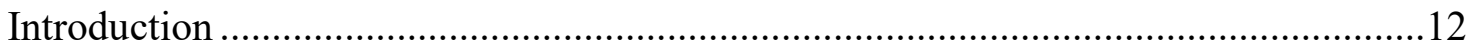

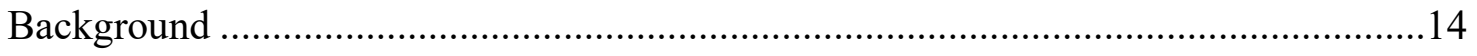

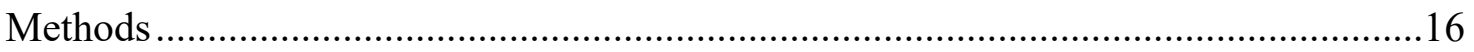

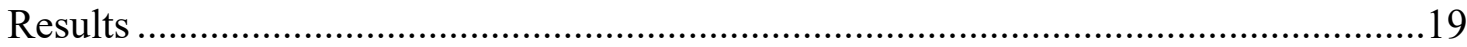

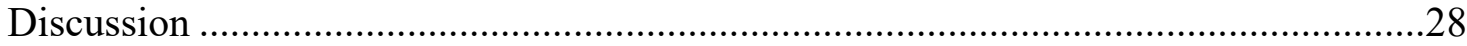

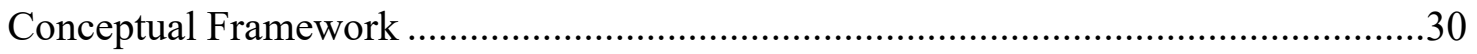

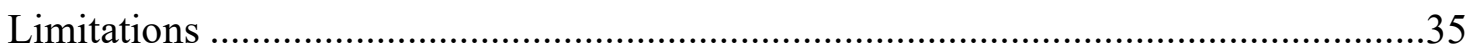

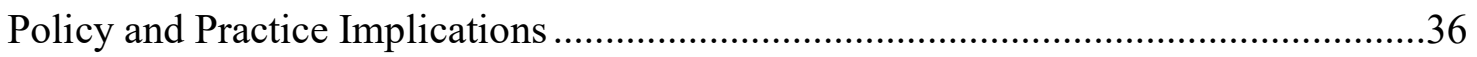

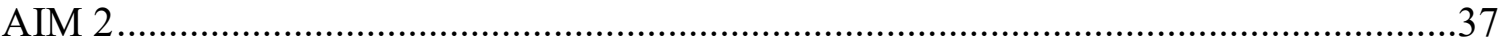

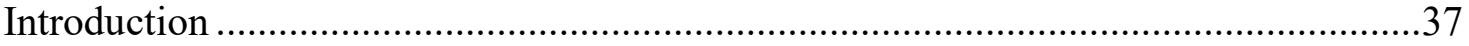

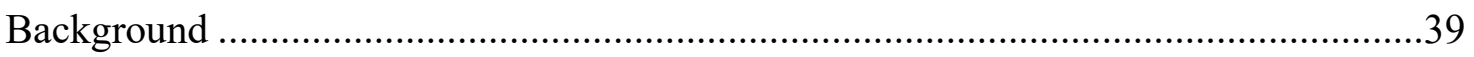

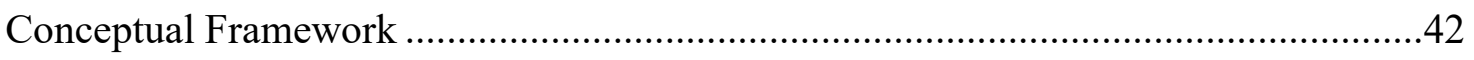

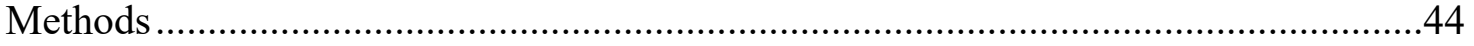

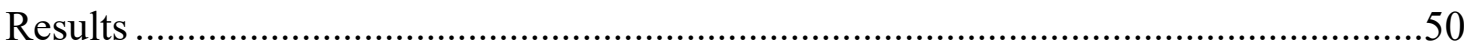

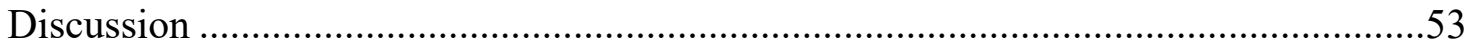

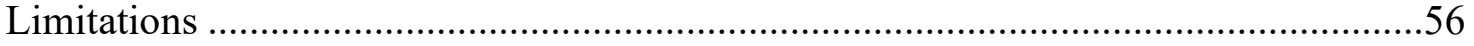




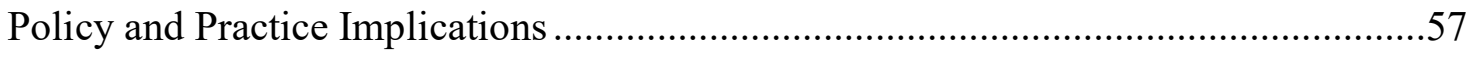

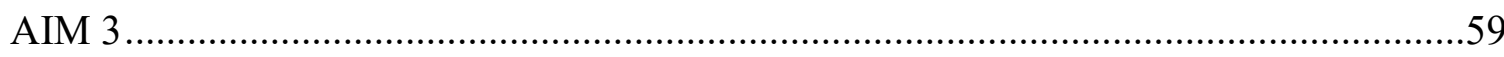

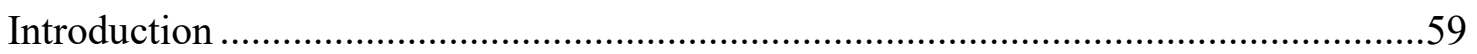

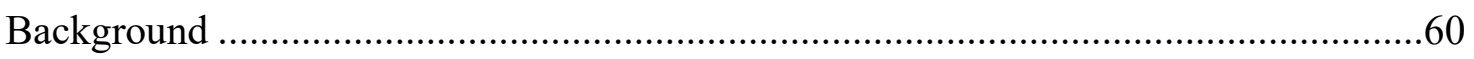

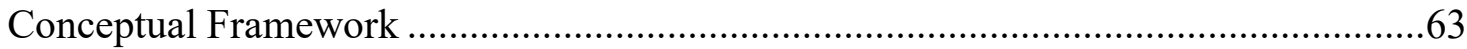

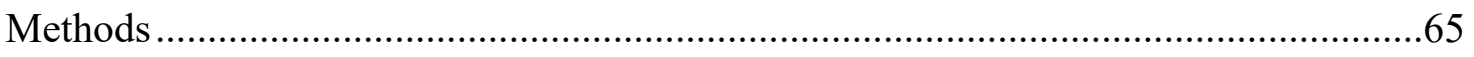

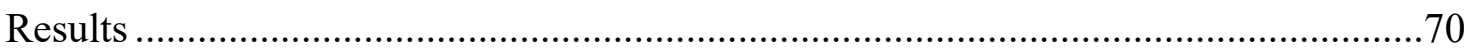

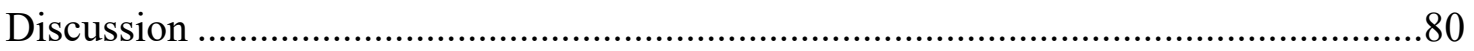

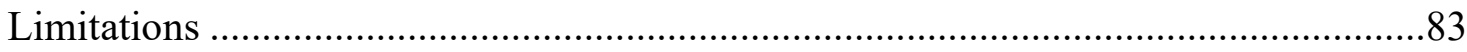

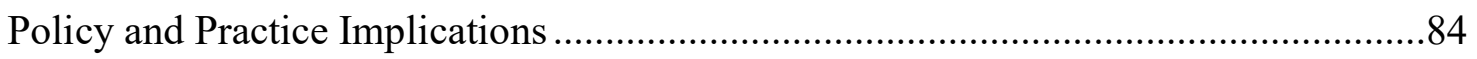

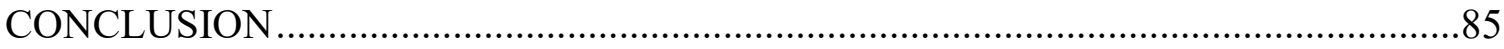

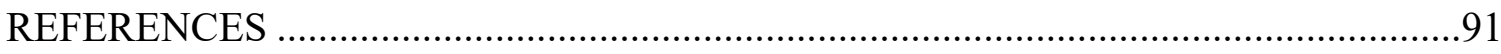

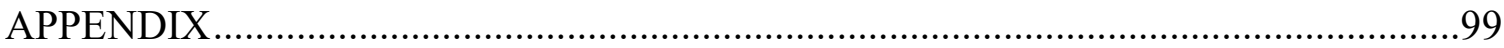

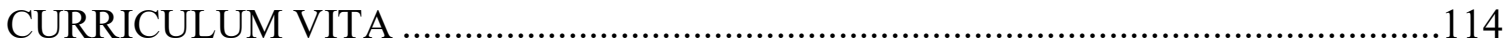




\section{LIST OF TABLES}

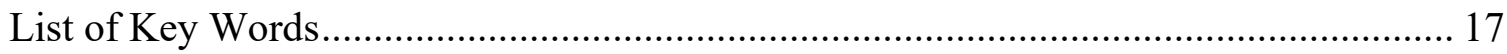

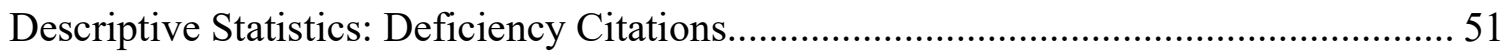

Results on the Number of Deficiency Citations ............................................................. 53

Descriptive Statistics: Falls, Bladder and Bowel Incontinence ........................................ 71

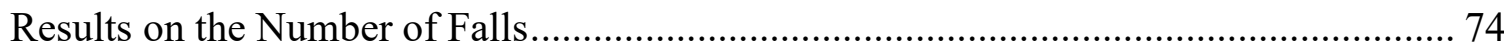

Results on the Number of Prevalence of Bladder Incontinence ……………………....... 77

Results on the Number of Prevalence of Bowel Incontinence ........................................... 80 


\section{LIST OF FIGURES}

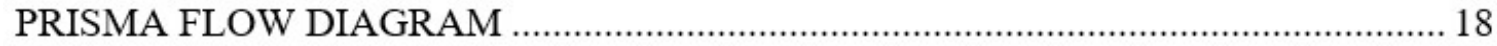

Conceptual Framework: GH Elements and their Impact ................................................ 31

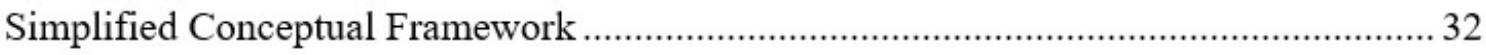

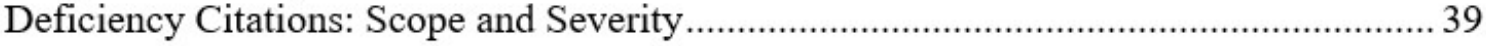

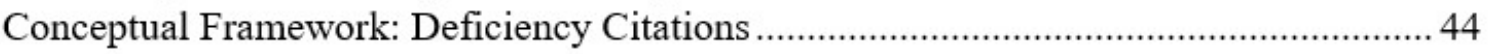

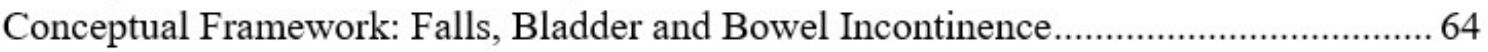

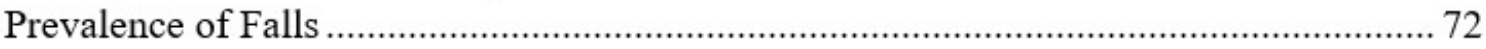

Prevalence of Bladder Incontinence ................................................................................. 75

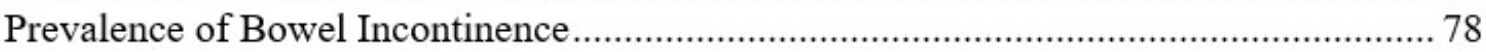




\section{CHAPTER 1}

\section{INTRODUCTION}

The long-term care field provides a wide range of services both at the personal and health levels. The field cares for individuals who are older, fragile, and cannot perform normal daily activities due to chronic diseases; injury; or disabilities at the mental, physical, or cognitive levels (U.S. Department of Health and Human Services (HHS), 2020). The long-term care field provides various services including the activities of daily living (ADLs) and the instrumental activities of daily living (IADLs) (Harris-Kojetin et al., 2019). The ADLs include dressing, bathing, and toileting services while the IADLs include medication management and housework activities (Harris-Kojetin et al., 2019). Therefore, each long-term care type is specific based on which activities they provide to the target population.

Assisted living facilities, nursing homes (NHs), adult day services center, and home health care are examples of the types of long-term care settings that provide an array of services based on the types of need (Pioneer Network, 2011; Harris-Kojetin et al., 2019). According to the Pioneer Network (2011), a NH is a facility which attends to a person who suffers from chronic conditions or have disabilities. Assisted living communities are defined as "facilities that provides or coordinates oversight and services to meet residents" individualized, scheduled needs, based on the residents' assessment and service plans, and their unscheduled needs as they arise.” (Pioneer Network, 2011). 
Adult day service centers are facilities that help family members in providing services to their senior relatives (Pioneer Network, 2011). These centers offer refreshments, social engagement, and other activities to individuals aged 65 who are disabled and over while their family members are working (Pioneer Network, 2011). Home health care agencies provide and coordinate services to individuals living at home (Pioneer Network, 2011).

These agencies employ registered nurses, licensed practical nurses, home health aides, or other certified professionals (Pioneer Network, 2011). In 2016, there were 15,600 NHs where approximately $83 \%$ of the population are NHs residents and aged 65 and over (Harris-Kojetin et al., 2019). In the same year, there were 4,600 adult day services centers and 286,300 individuals were using the services (Harris-Kojetin et al., 2019). In 2015, 12,200 home health agencies and 4,455,700 individuals were discharged from these agencies (Harris-Kojetin et al., 2019). In 2016, 811,500 persons resided in 28,900 residential care communities (Harris-Kojetin et al., 2019). Based on these statistics, one can say that the long-term care field provides a range of services to people who are aged 65 and older.

For the purpose of our research, we focused on the NHs. According to the Pioneer Network (2011), NHs provide 24-hour care to residents who experience chronic diseases or have disabilities. The population residing in NHs cannot care for themselves and are in need of consistent help at the medical level and/or skilled nursing care (Pioneer Network, 2011). Traditional NHs have been considered as institutions with a hospital-like environment (Yoon, Brown, Bowers, Sharkey, \& Horn, 2015) where residents are more 
likely to be abused, be alone, or isolated (Cohen et al., 2016). Normally, an institution as such should care for its residents with high quality. Additionally, individuals admitted to NHs usually suffer from four main conditions including bladder incontinence, depression, Alzheimer's-type dementia, and bowel incontinence (Shi \& Singh, 2015, p.400). Family members having their loved-ones in NHs created awareness around the issues experienced by the NH residents in 1980s, and in 1987, a policy called "the Omnibus Reconciliation Act" (OBRA-87) was passed that focused on the improvement of the quality of care of NHs (Bowers, Nolet, \& Jacobson, 2016a; Cohen et al., 2016).

From the policy emerged various components that provoked a change in the longterm care field including the Long Term Care Minimum Data Set (MDS), the deficiency citations, and the culture change movement (Miller et al., 2013; Cohen et al., 2016). The CMS required facilities that received Medicaid and/or Medicare reimbursement to report quality of care indicators such as the prevalence of falls, the prevalence of bladder/bowel incontinence, the prevalence of pressure ulcers, the prevalence of urinary tract infection (UTI), and other indicators (Cohen et al., 2016). In addition, facilities are surveyed once a year (Castle, Wagner, Ferguson, \& Handler, 2011a). Once a facility is reported to not be aligned with the CMS requirements, then the facility receives a deficiency citation (Mukamel et al., 2007).

We decided to focus on the prevalence of bladder and bowel incontinence since they are two of the four main conditions that $\mathrm{NH}$ residents are more likely to experience (Shi \& Singh, 2015, p.400). Additionally, we focused on the number of falls since they are prevalent in NH facilities (Fritsch et al., 2009; Shura, Siders, \& Dannefer, 2011; Bourgeois, Brush, Elliot, \& Kelly, 2015; Hermer, Bryant, Pucciarello, Mlynarczyk, \&Zhong, 2017). 
Lastly, our research was also interested in deficiency citations since they are used as quality indicators in the NH literature (Temkin, Zheng, Cai, Zhao, \& Mukamel, 2010; Grabowski, Elliot, Leitzell, Cohen, \& Zimmerman 2014a).

\section{Falls}

Three million older individuals have emergency department visits due to fall injuries every year (as cited in the Centers for Disease Control and Prevention (CDC), 2017). Hospitalizations occurred in more than 800,000 individuals due to fall injuries, head injury, or hip fracture (as cited in CDC, 2017). Each year, hospitalizations occurred in nearly 300,000 older individuals because of hip fractures (as cited in CDC, 2017) with 95 percent of hip fractures caused by falling (as cited in CDC, 2017). In the literature, falls risk factors were identified and included weakness, vitamin D deficiency, inconsistencies with physical balance, using medical drugs, vision issues, and home obstacles (CDC, 2017). In most cases, individuals who fall experience more than one risk factor (CDC, 2017). Fall prevention consists of visiting with the doctor to discuss solutions to manage known risk factors, i.e. taking medicines that are known, vitamin D supplements, exercising, vision exams, and removing obstacles at home (CDC, 2017).

\section{Bladder and Bowel Incontinence}

Bladder and bowel incontinence are disorders experienced mostly by older individuals and can lead to severe consequences in their quality of life and healthcare services (Gorina et al., 2014). Additionally, bladder and bowel incontinence are two of the main conditions that NH residents encounter (Shi \& Singh, 2015, p.400). Bladder incontinence was defined as a condition where: "the resident reported as not having an indwelling catheter and not 
in complete control of urinary bladder function during 14 days prior to the assessment" (Gorina et al., 2014).

Bowel incontinence was defined as a condition where: "a resident reported as no having an ostomy and not in complete control of bowel movement during 14 days prior to the assessment" (Gorina et al., 2014). A report from four national datasets including National Health and Nutrition Examination Survey (NHANES), National Survey of Residential Care Facilities (NSRCF), National Home and Hospice Care Survey (NHHCS), and MDS presented data on the prevalence of bladder and bowel incontinence (Gorina et al., 2014). According to the report, approximately 46.1 percent of short-term NH residents and 75.8 percent of long-term residents were bladder or bowel incontinent during the 14 days prior to the interview (Gorina et al., 2014). The same report described that over 36.7 percent of short-term NH residents and 70.3 percent of long-term residents were bladder incontinent while 33.1 percent of short-term and 60.0 percent of long-term residents experienced bowel incontinence (Gorina et al., 2014).

\section{Deficiency Citations}

Deficiency citations are given to facilities that are not aligned with the CMS requirements (Castle, Engberg, \& Men, 2007). Nursing homes that receive Medicaid or Medicare reimbursement are required to go through a certification process (Castle, 2011b). The certification process is established by the federal government to regulate the quality of care for residents living in NHs (Castle et al., 2007). The federal government is the primary payer of NH care and manages quality care through Medicaid and Medicare (Castle et al., 2007). NHs are required to meet the minimum standards which were created as a part of Titles XVIII and XIX of the Social Security Act (Castle et al., 2007). 
A deficiency citation is identified by the scope and severity of the issue (Castle et al., 2011a). Twelve categories exist from A to L, which assess the gravity of the deficiency (Castle et al., 2011a). The A category is defined as the least severe while $\mathrm{L}$ is determined as the most severe (Castle et al., 2011a). Severity is defined as the level of harm experienced by the resident while scope is the total number of residents who suffered such abuse with isolated cases, a pattern of cases, and widespread cases (Castle et al., 2011a). The certification process takes place nearly every year with an inspector team that inspect the NH facilities (Castle et al., 2011a).

Quality of care is surveyed during the periodic inspections and the surveyors decide if the facilities have met the requirements set by the CMS (Castle et al., 2011a). Once a facility fails to meet the requirements, fines are applied depending on the degree of the issue. Facilities can receive up to $\$ 10,000$ daily and can experience closure by the Medicare/Medicaid programs (Castle, 2011b). According to the Department of Health and Human Services (DHHS) (2004), a deficiency citation is defined as "a finding that a nursing home failed to meet one or more federal or state requirements." According to a 2019 DHHS report, 94 percent of deficiencies were less severe (labels D, E, F) compared to 6 percent of deficiencies that were more severe (labels G-L, or with $\mathrm{F}$ with a poor quality) (DHHS, 2019). Based on the DHHS report, an increase in the number of deficiencies was observed from 2013 to 2016, then a decrease in the number of deficiencies was observed in 2017.

Earlier, we discussed the various components which derived from the OBRA-87 policy including the MDS, deficiency citations, and the culture change. So far, we discussed the quality indicators found in the MDS, and the background and process of the 
deficiency citations. This research study focuses mainly on the culture change movement, and its recent model known as the Green House (GH) model. The culture change movement originated out of the OBRA-87 with the intention of deinstitutionalizing the long-term care field and centralizing on resident care (Koren, 2010). Culture change covers six domains: "resident-directed care and activities; a homelike environment; close relationships among staff, family, residents, and community; staff empowerment; collaborative and decentralized management; and measurement-based continuous quality improvement" (Koren, 2010).

\section{The Culture Change Movement}

Regarding the implementation of culture change, variations are observed across NHs. Some NHs adopt the full model of culture change, while others implement certain concepts of the model, and others, i.e. the traditional NHs, do not implement it at all. Before 2007, a Commonwealth Fund survey showed that 73 percent of NH leaders were not aware of the culture change movement (Koren, 2010). However, in 2008, only 34 percent of the leaders did not know about the movement (Koren, 2010). Also, a 2007 Commonwealth Fund survey reported that 56 percent of NHs acknowledged that they adopted some factors related to culture change (Bowers et al., 2016a). This percentage increased to 85 percent in a 2009-2010 survey (Bowers et al., 2016a).

In the past, NHs have implemented various types of culture change models including Wellspring, Planetree, the Eden Alternative, and the GH model with the goal of improving the quality of care in NHs (Cohen et al., 2016; Miller, Mor, \& Burgess, 2016). The Wellspring model focuses on transforming the traditional setting into a more residentcentered environment and allows its employees to make decision (Cheek, Nolan, \& Larsen- 
Orta, 2008). The Planetree was first adopted in hospitals and was extended to the long-term care field (Cheek et al., 2008). The Planetree model is defined as: "a holistic approach that encourages healing in several dimensions including mental, emotional, spiritual, social, and physical" (Cheek et al., 2008). This model seeks to transform the physical structure into a more home-like atmosphere (Cheek et al., 2008). The Eden Alternative was created by Dr. William Thomas in 1991 (Hill, 2011). The model objective is to transform the NH into a more approachable environment where family members especially children are allowed to visit, and animals and plants are permitted as well (Hill, 2011). The GH model was also created by Dr. William Thomas and this model is often considered as the sister of the Eden Alternative (Hill, 2011). For the purpose of our research, we will focus on the GH model.

\section{Background on the GH Model}

In 2003, the GH model was created with a focus on the deinstitutionalization of long-term care and person-centered care (Rabig, Thomas, Kane, Cutler, \& McAlilly, 2006). The GH model consists of a small home with up to 12 residents who have access to a private room, bathroom, and a common area for dining purposes (Afendulis, Caudry, O'Malley, Kemper,

\& Grabowski, 2016; Cohen et al., 2016; Grabowski et al., 2016; Rabig et al., 2006; Zimmerman et al., 2016). GH homes have increased from four homes in 2003 to 174 homes in 2015 (Lum, Kane, Cutler, \& Yu, 2008; Zimmerman et al., 2016). Currently, there are $237 \mathrm{GH}$ homes that are in operation and $60 \mathrm{GH}$ homes are in development in 31 states ("Find a home", 2020). Some GH homes share a license with a traditional NH (called legacy homes), while others have their own license (Afendulis et al., 2016). 
A GH home uses various types of staffing that differs from that used in traditional NHs. GH staff often include Shahbazim, a Guide, a Sage, and other non-clinical staff (Ragsdale \& McDougall, 2008). The Shahbazim are the equivalent of certified nursing assistants (CNAs) in traditional NHs (Afendulis et al., 2016). However, the Shahbazim have more tasks than do traditional CNAs (Brown et al., 2016), and are considered "universal caregivers" (Cohen et al., 2016). Shahbazim are responsible for preparing meals, doing laundry, housekeeping, and supporting residents' personal care and overall quality of life (Brown et al., 2016; Cohen et al., 2016; Rabig, et al., 2006). Shahbazim have a manager called a Guide who oversees the Shahbazim work (Rabig et al., 2006). A Sage is a community member carefully chosen by the GH Guide and staff, who volunteers to provide advice to the Shahbazim and support them in regard to decision-making and problem-solving duties (Ragsdale \& McDougall, 2008). A group of social workers, dieticians, nurses, and activity personnel stays near to the GH homes so they can provide services to the residents (Rabig et al., 2006; Ragsdale \& McDougall, 2008). Thus, personcenteredness, small homes, room privacy, and a special team of employees are some of the key elements of the GH model.

In the literature, there is a mix of findings related to the overall impact of the $\mathrm{GH}$ model on quality of care and organizational functioning. One study reported that the implementation of the GH model was associated with a decrease in 30-day readmission rates, bedfast residents, catheterized residents, and pressure ulcers among low-risk residents (Afendulis et al., 2016). Brown et al. (2016) found a lower turnover of staff in GH homes compared to traditional NHs. Shahbazim were also independent in terms of scheduling their own agenda compared to CNAs in traditional NHs (Brown et al., 2016). 
The same study showed that the culture of safety, staff stress, and staff satisfaction were not negatively affected by increased staff autonomy (Brown et al., 2016). Lastly, others found that the model lowered Medicare Part A spending (Grabowski et al., 2016).

Contrarily, other studies found that the GH model negatively impacted quality of care. Problems related to management, collaboration in decision-making, and training skills for the management team were observed (Bowers et al., 2016a). Daily minor issues and problems related to expertise growth for the staff were also highlighted in the literature (Bowers et al., 2016a). One of the objectives of the model was to promote more collaboration between the staff and the management team especially during decisionmaking, but research showed that the model did not deliver (Bowers et al., 2016a). Brown et al. (2016) stated that, while there was a lower turnover of staff, the Shahbazim had a heavy workload compared to the CNAs in traditional NHs (Brown et al., 2016).

However, a gap still exists in the literature in terms of empirically analyzing how specific elements of the GH model impact quality of care. Zimmerman and Cohen (2010) reported in their study that a consensus has been reached that the six key elements of the GH model include physical structure, staffing, dining, resident-case mix, resident-centeredness, and engagement (Zimmerman \& Cohen, 2010). Cohen et al. (2016) and Zimmerman et al. (2016) stressed the importance of identifying which element has an impact on quality of care. Therefore, it is important to study the design of the GH model and identify which GH components influence outcomes. Our study includes three aims which are stated below: Specific Aim 1: To conduct a systematic review to understand the design and effects of the GH model by using the Preferred Reporting Items for Systematic Reviews and Meta- 
Analyses (PRISMA) approach and a conceptual model. What is the impact of the GH model in NHs that adopted the model?

Specific Aim 2: To investigate the impact of the GH model on deficiency citations in NHs.

Do GH homes have less deficiency citations than non-GH homes?

Specific Aim 3: To assess the impact of the GH model on the indicators of quality of care in GHNHs. Which GH model elements have an effect on falls, bladder incontinence, and bowel incontinence in $\mathrm{NH}$ residents living in GH facilities and what are these effects? 


\section{CHAPTER 2: AIM 1}

The Green House Nursing Home Model: A Systematic Review of Model Elements and their Impact

More than 1.4 million individuals lived in nearly 16,000 nursing homes (NHs) in 2012 (Centers for Medicare and Medicaid Services [CMS], 2013). Each year, approximately three million people are admitted in NHs (Shier, Khodyakov, Cohen, Zimmerman, \& Saliba, 2014). The number of NH residents is estimated to increase with the growth of individuals over 65 years old. In fact, currently, the total number of people who are 65 years old and above is 49 million people which is estimated to rise to 98 million people in 2060 (Administration on Aging, 2018). Therefore, the future demand for NHs services is likely to experience an exponential growth.

With this foreseeable demand, it is crucial to understand the NHs' advantages and disadvantages. As an organization that structures relations between staff, residents, and residents' families, a NH can be empirically analyzed through the lenses of organizational theory. Scott and Davis (2007) described three basic levels at which organizations can be studied: social psychological, organizational and ecological. For the purpose of this research, the focus will be at the organizational level, wherein, according to Scott and Davis (2007), the researcher attempts to examine the structure and social processes of an organization and its components. 
Ideally, a NH should be a welcoming place where rest and calm are prioritized. The NH should also be a place where residents receive care but, in some cases, it has been the opposite. NH residents often suffer from cognitive impairment, falls, psychological/behavioral issues, bowel and bladder incontinence, and pressure ulcers (Ausserhofer et al., 2016; Horn et al., 2016; Konetzka, Brauner, Shega, \& Werner, 2014). In addition to these conditions, NHs have been described as institutions where residents experience isolation, untreated pain, abuse, depression, and lack of decency (Ausserhofer et al., 2016; Brune, 2011; Cohen et al., 2016).

In the 1980s, a consumer advocacy group raised awareness on the issues experienced by NH residents (Koren, 2010). In response to NH issues, the Nursing Home Reform Act was passed as part of the Omnibus Reconciliation Act of 1987 (OBRA-87) (Bowers et al., 2016a; Cohen et al., 2016), which focused on decreasing the likelihood of poor quality of care experienced by NH residents (Cohen et al., 2016). Following OBRA87, a new movement referred to as culture change was initiated with the objective of deinstitutionalizing long-term care and focusing efforts on improving resident care (Bowers et al., 2016a; Koren, 2010). One facet of the culture change movement was the development of the GH model (Bowers et al., 2016a, Cohen et al., 2016). Other types of culture change models include the Eden Alternative, the Pioneer Network, the Meadowland Hills Program, Wellspring, and Planetree (Cohen et al., 2016; Ragsdale \& McDougall, 2008). For the purpose of this systematic review, we will focus on the GH model with an attempt to answer the following research question: "What is the effect of the GH model in NHs that adopted the model?" Our three study objectives are to: (1) investigate the GH elements that have been studied in the literature; (2) identify which elements of the GH 
model impact the quality of care of residents; and (3) identify which organizational theory was used in the analysis of the GH model on quality of care.

\section{BACKGROUND}

In 2003, the GH model was created with a focus on the deinstitutionalization of long-term care and person-centered care (Rabig et al., 2006). The GH model consists of a small home with up to 12 residents who have access to a private room, bathroom, and a common area for dining purposes (Afendulis et al., 2016; Cohen et al., 2016; Grabowski et al., 2016; Rabig et al., 2006; Zimmerman et al., 2016). GH homes have increased from four homes in 2003 to 174 homes in 2015 (Lum et al., 2008; Zimmerman et al., 2016). Currently, there are $237 \mathrm{GH}$ homes that are in operation and $60 \mathrm{GH}$ homes are in development in 31 states ("Find a home", 2020). Some GH homes share a license with a traditional NH (called legacy homes), while others have their own license (Afendulis et al., 2016).

A GH home uses various types of staffing that differs from that used in traditional NHs. GH staff often include Shahbazim, a Guide, a Sage, and other non-clinical staff (Ragsdale \& McDougall, 2008). The Shahbazim are the equivalent of certified nursing assistants (CNAs) in traditional NHs (Afendulis et al., 2016). However, the Shahbazim have more tasks than do traditional CNAs (Brown et al., 2016), and are considered "universal caregivers" (Cohen et al., 2016). Shahbazim are responsible for preparing meals, doing laundry, housekeeping, and supporting residents' personal care and overall quality of life (Brown et al., 2016; Cohen et al., 2016; Rabig, et al., 2006). Shahbazim have a manager called a Guide who oversees the Shahbazim work (Rabig et al., 2006). A Sage is a community member carefully chosen by the GH Guide and staff, who volunteers to provide advice to the Shahbazim and support them in regard to decision-making and 
problem-solving duties (Ragsdale \& McDougall, 2008). A group of social workers, dieticians, nurses, and activity personnel stays near to the GH homes so they can provide services to the residents (Rabig et al., 2006; Ragsdale \& McDougall, 2008). Thus, personcenteredness, small homes, room privacy, and a special team of employees are some of the key elements of the GH model.

In the literature, there is a mix of findings related to the overall impact of the GH model on quality of care and organizational functioning. One study reported that the implementation of the GH model was associated with a decrease in 30-day readmission rates, bedfast residents, catheterized residents, and pressure ulcers among low-risk residents (Afendulis et al., 2016). Brown et al. (2016) found a lower turnover of staff in GH homes compared to traditional NHs. Shahbazim were also independent in terms of scheduling their own agenda compared to CNAs in traditional NHs (Brown et al., 2016). The same study showed that the culture of safety, staff stress, and staff satisfaction were not negatively affected by increased staff autonomy (Brown et al., 2016). Lastly, others found that the model lowered Medicare Part A spending (Grabowski et al., 2016).

Contrarily, other studies found that the GH model negatively impacted quality of care. Problems related to management, collaboration in decision-making, and training skills for the management team were observed (Bowers et al., 2016a). Daily minor issues and problems related to expertise growth for the staff were also highlighted in the literature (Bowers et al., 2016a). One of the objectives of the model was to promote more collaboration between the staff and the management team especially during decisionmaking, but research showed that the model did not deliver (Bowers et al., 2016a). Brown 
et al. (2016) stated that, while there was a lower turnover of staff, the Shahbazim had a heavy workload compared to the CNAs in traditional NHs (Brown et al., 2016).

However, a gap still exists in the literature in terms of empirically analyzing how specific elements of the GH model impact quality of care. Zimmerman and Cohen (2010) reported in their study that a consensus has been reached that the six key elements of the GH model include physical structure, staffing, dining, resident-case mix, resident-centeredness, and engagement (Zimmerman \& Cohen, 2010). Cohen et al. (2016) and Zimmerman et al. (2016) stressed the importance of identifying which element has an impact on quality of care. Therefore, it is important to study the design of the GH model and identify which GH components influence outcomes.

\section{METHODS}

We analyzed the literature according to the guidelines of the Preferred Reporting Items for Systematic Reviews and Meta-Analyses (PRISMA) (Moher, Liberati, Tetzlaff, Altman, \& the PRISMA Group, 2009). We searched five databases including PubMed, Embase, EBSCO Host, Medline (Ovid), and Web Science. We combined these following key words: "greenhouse model”, "nursing home”, “organizational theory", “citation deficiency", “culture change", "falls", "pressure ulcers", “bipolar condition", “incontinence”, and “quality indicators" (see Table 1). 


\section{Table 1.}

$\underline{\text { List of key words }}$

\begin{tabular}{ll}
\hline 1. & Green house model \\
\hline 2. & Green house model AND nursing home \\
\hline 3. & 2 AND organizational theory \\
\hline 4. & 2 AND citation deficiency \\
\hline 5. & 2 AND culture change \\
\hline 6. & Green house model AND long-term care \\
\hline 7. & Culture change AND long-term care \\
\hline 8. & 2 AND falls \\
\hline 9. & 2 AND incontinence \\
\hline 10. & 2 AND quality indicators \\
\hline 11. & Culture change AND nursing home \\
\hline 12. & 11 AND organizational theory \\
\hline 13. & 11 AND citation deficiency \\
\hline 14. & 11 AND quality indicators \\
\hline 15. & 11 AND falls \\
\hline 16. & 11 AND incontinence \\
\hline
\end{tabular}

Table 1

Figure 1 shows the number of articles that were included at each step of the review. First, we screened for titles based on our research objectives before then reviewing the abstracts of the remaining articles. We included peer-reviewed articles that were published in the U.S. since the GH model first emerged in 2003 (Rabig et al., 2006).

We excluded book reviews, editorials, conference presentations, e-books, and articles that did not provide enough information on the GH model. Last, we read the fulltext of the remaining articles, which all focused on culture change. Culture change articles where the GH model was presented only as an example were excluded. For the selected 
articles, information was extracted on the study designs, sample size, and outcomes (View Appendix).
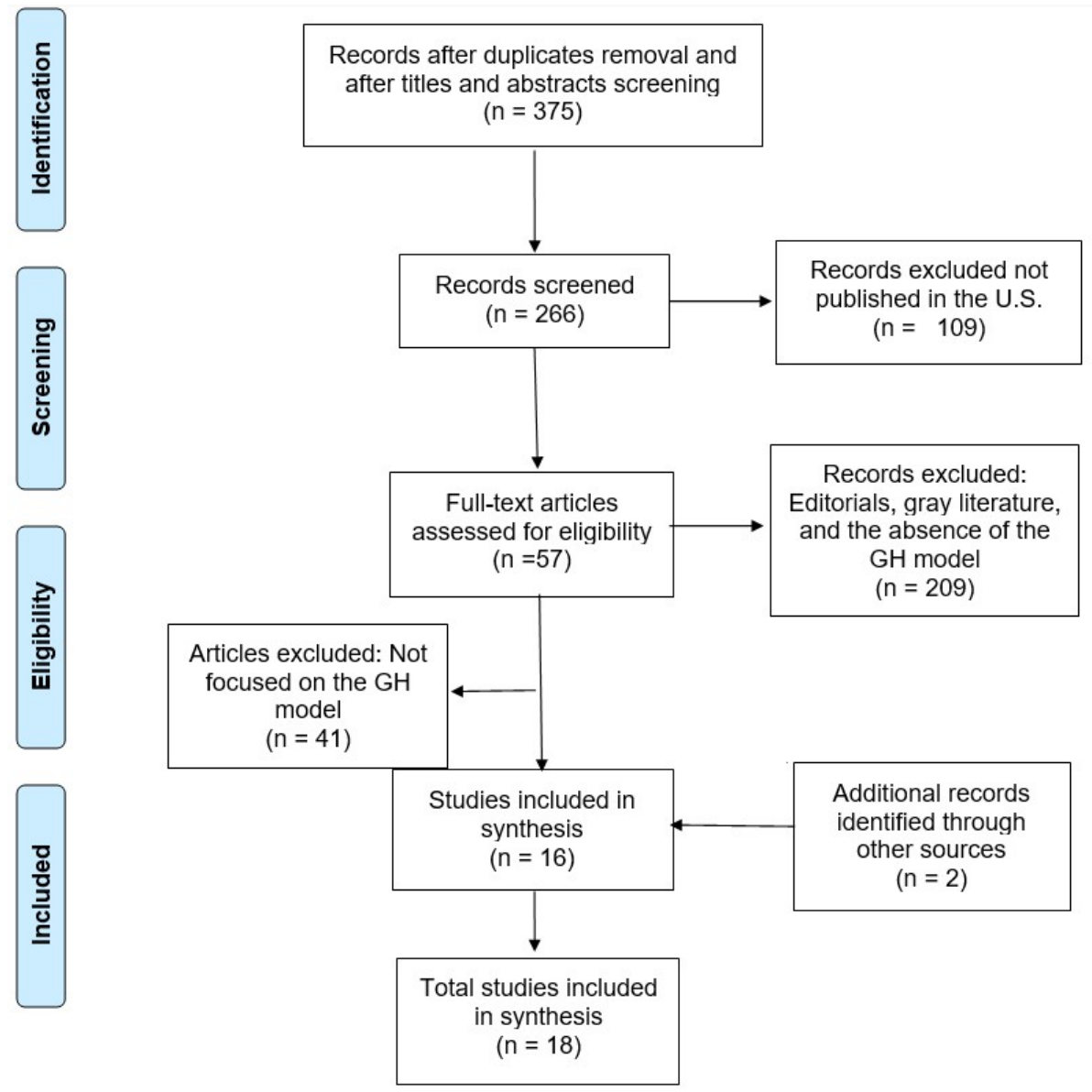

PRISMA FLOW DIAGRAM

Figure 1. PRISMA Flow Diagram

After removing those articles and duplicates, 375 articles remained across the five databases. From the 375 articles, 266 articles remained that were published in the U.S. Our next step was to read the full-text articles where 56 articles were kept and 210 articles were excluded. The reason for exclusion was because some articles did not discuss the GH model while others were editorials, e-book, or other types of gray literature.

The inclusion criteria was the fact that the articles were focused on the culture change movement. Out of the 56 articles, 16 articles were kept and 41 articles were 
removed. The 16 articles focused on the GH model while the 41 articles focused on the culture change movement and presented the GH model as an example. In addition to the 16 articles, we added two articles that we identified from the other articles' reference list. A second person reviewed the articles that were selected for the analysis. In total, we analyzed 18 articles. Figure 1 shows the number of articles that we excluded or included at each level of the analysis.

\section{RESULTS}

We categorized our results into two main groups based on our two first research objectives: (1) to investigate the GH elements that have been studied in the literature, and (2) to identify which elements of the GH model impact quality of care of the NH residents. The main group called GH model design incorporates studies that analyzed the GH model overall and the GH elements, i.e. (1) the physical structure; (2) staffing; (3) residentcenteredness; (4) engagement; and (5) family involvement. The second main group named the GH model and quality of care includes studies that analyzed the GH model overall and the GH elements, i.e. the physical structure and staffing.

\section{GH Model - Design}

\section{GH Model Overall}

One article mainly provided a description of the GH homes (Grabowski et al., 2014a). GH homes were more likely to be part of a nonprofit organization compared to other NHs (traditional and culture change NHs) (Grabowski et al., 2014a). The study also reported that at baseline, GH homes were more likely to have private-payers and an increase in nursing assistant-staffing per resident day (Grabowski et al., 2014a). 


\section{GH Elements}

Five elements were analyzed including the physical structure, staffing, residentcenteredness, engagement, and family involvement. Four studies assessed the physical structure of the GH model (Bowers, Roberts, Nolet, \& Ryther, 2016b; Cohen et al., 2016; Horn et al., 2016; Jenkens, Sult, Lessell, Hammer, \& Ortigara, 2011) while six articles focused on staffing (Bowers \& Nolet, 2011; Bowers et al., 2016a; Brown et al., 2016; Cohen et al., 2016; Loe \& Moore, 2012; Sharkey, Hudak, Horn, James, \& Howes, 2011). Cohen et al. (2016) studied the resident-centeredness and three studies focused on engagement (Cohen et al., 2016; Kane, Lum, Cutler, Degenholtz, \& Tzy-Chyi, 2007; Yoon et al., 2015). Lum et al. (2008) discussed on family involvement as part of the GH model elements.

\section{Physical Structure}

Four studies analyzed physical structure in terms of number of beds, small scale, private area, a home-like structure, location, and cost (Bowers et al., 2016b; Cohen et al., 2016; Horn et al., 2016; Jenkens et al., 2011). One study reported that the size of the GH homes (mean of 10.2 beds) is smaller in comparison to traditional NHs (mean of 34.4 beds) (Horn et al., 2016). The physical structure of the GH model creates a homelike atmosphere where residents, their families and friends, and the staff can gather (Bowers et al., 2016b).

Additionally, secured outdoor areas were found to be higher in GH homes (75 percent) compared to traditional NHs (33 percent) (Cohen et al., 2016). Only 36 percent of the rooms in legacy NHs were private compared to rooms in all GH homes, i.e. 100 percent (Cohen et al., 2016). Jenkens et al. (2011) found that GH homes are expensive to build compared to traditional NHs. For a GH home, the cost per bed is $\$ 83,200$ per resident for 
a 650 square feet per resident while for a traditional $\mathrm{NH}$, the cost per bed for a private room is $\$ 40,704$ per resident for 318 square feet per resident and the cost per bed for a semiprivate room is $\$ \$ 30,592$ per resident for a 239 square feet per resident (Jenkens et al., 2011). The authors based their calculations of the national average of $\$ 128$ per square foot (Jenkens et al., 2011).

\section{Staffing}

Six articles analyzed staffing as one of the GH elements (Bowers \& Nolet, 2011; Bowers et al., 2016a; Brown et al., 2016; Cohen et al., 2016; Loe \& Moore, 2012; Sharkey et al., 2011). Universal role within self-managed teams was identified as a sub-element (Cohen et al., 2016). Self-managed teams were present in all the GHs but absent in traditional NHs (Cohen et al., 2016). Even though self-managed teams are described as a positive component, the study reported that there was a scheduling issue among the Shahbazim due to the fear of responsibility (Cohen et al., 2016).

In regard to the universal role sub-element, the staff performed different activities in the GH homes compared to traditional NHs (Cohen et al., 2016). For example, 100 percent of staff in GH homes prepared meals for the residents compared to 0 percent of staff in traditional NHs (Cohen et al., 2016). One hundred percent of staff in GH homes did the laundry compared to eight percent of staff in traditional NHs (Cohen et al., 2016). In addition, 75 percent of the staff in the GH homes worked on housekeeping work compared to 0 percent of staff in traditional NHs (Cohen et al., 2016). One hundred percent of GH home staff assisted residents with meals compared to 75 percent of traditional NHs staff (Cohen et al., 2016). Additionally, 75 percent of GH home staff assisted the residents with exercising activities compared to 67 percent of traditional NH staff (Cohen et al., 
2016). Concerning the same sub-component, the same study found that there were variations among GH homes in regard to staff activities (Cohen et al., 2016). For example, in 64 percent of GH homes, staff performed outing activities with the residents while in other GH homes, the activities were not common or non-existent (Cohen et al., 2016).

Another sub-theme reported in the literature was the empowerment of the staff in GH homes. Transitioning from CNAs to Shahbazim, Loe and Moore (2012) found that the staff who were empowered experienced a decrease in stress in their work environment and an improvement in their relationships with the residents, and improved on their control of time and space. However, challenges were present related to staff empowerment and include working individually, conflicts between team members, and accepting responsibility (Bowers \& Nolet, 2011). One study reported that GH homes were struggling in maintaining the elements of the GH model; as a result, some GH homes went back to a traditional NH while others maintained their new ways (Bowers et al., 2016a). One of the GH elements that the GH homes maintained was the empowerment of the staff (Bowers et al., 2016a). Bowers and Nolet (2011) discussed that the staff (especially Shahbazim) were pleased with their tasks and felt that nurses respected them. However, other Shahbazim were not satisfied as much and others did not find any difference between GH homes and traditional NHs (Bowers \& Nolet, 2011).

Two studies reported on staff salary and their time spent working (Brown et al., 2016; Sharkey et al., 2011). Total nursing hours differed between GH homes (5.3) and traditional NHs (3.6) (Sharkey et al., 2011). Shahbazim had 1.56 more total nursing hours compared to CNAs in traditional NHs (Sharkey et al., 2011). However, non-nursing total hours were lower (2 hours less) in GH homes compared to traditional NHs (Sharkey et al., 
2011). Brown et al. (2016) compared the characteristics of the staff (Shahbazim, licensed practical nurses (LPNs), and registered nurses (RNs)) in GHs and traditional NHs. They found that Shahbazim in GH homes are paid $\$ 0.60$ per hour more on average than CNAs in legacy homes (Brown et al., 2016). Also, turnover rates were low for CNAs and LPNs in GH homes compared to non-GH NHs (Brown et al., 2016).

\section{Resident-Centeredness}

Cohen et al. (2016) reported on resident-centeredness as one of the GH elements. Having a meaningful life contributed to resident control, decision making, and engagement (Cohen et al., 2016). Compared to traditional NHs, GH home residents had more choice in waking up (67 percent vs. 8 percent), bed ( 83 percent vs. 8 percent), and bath times (33 percent vs. 8 percent) (Cohen et al., 2016). However, some GH homes constrained residents in their choices in regard to wake-up-, bed-, and bath-times (Cohen et al., 2016). Cohen et al. (2016) also reported that GH homes (75 percent) were more likely to approve residents and their families to use the open kitchen compared to traditional NHs (25 percent).

\section{Engagement}

Three studies measured how residents socially engaged in GH homes (Cohen et al., 2016; Kane et al., 2007; Yoon et al., 2015). GH home staff less often imposed activities on residents, instead allowing residents to engage in their own activities (Cohen et al., 2016). The same study reported that only 33 percent of the GH homes arranged activities in advance, compared to 100 percent of the legacy NHs (Cohen et al., 2016).

Yoon et al. (2015) found that GH residents needed improvement with social engagement. GH home residents were not engaged in social events similarly to residents 
in traditional NHs; however, the situation was not at the same rate (Yoon et al., 2015). GH home residents experienced isolation at a lower rate (with an estimate of $\beta=-0.274$ ) compared to traditional residents over time (Yoon et al., 2015). Another study found that social engagement was not present in GH homes (Kane et al., 2007).

\section{Family Involvement}

Lum et al. (2008) found that family members were not involved as much in some tasks such as laundry and shopping in GH homes compared to families in traditional NHs. However, family members were slightly more satisfied with GH homes compared to families in traditional NHs, though the difference in satisfaction was not statistically significant (Lum et al., 2008).

\section{GH Model - Quality of Care}

Ten studies assessed the impact of the GH model on quality of care. The results were categorized into two groups where one group reported on the impact of the GH model (without specifying the GH elements) on quality of care and the second group focused on the impact of the specific GH elements on quality of care.

\section{GH model Overall}

Seven studies analyzed preventable hospital readmissions (Afendulis et al., 2016), Medicare Part A (plus hospice) utilization (Grabowski et al., 2016), psychological symptoms (Yoon et al., 2015), quality measures as outcomes (Afendulis et al., 2016; Kane et al., 2007; Yoon, Brown, Bowers, Sharkey, \& Horn, 2016), urinary tract infection (UTI) rates (Wolff, Hassett, \& Kelly; 2016). Afendulis et al. (2016) discussed the impact of the GH model on hospitalization rates where the authors found a decrease in overall hospitalizations in GH homes compared to non-GH NHs with 1.3 percentage points to 0.9 
percent points. Additionally, Afendulis et al. (2016) reported a decrease of 3.9 percentage point in preventable hospitalization readmissions in GH homes. Grabowski et al. (2016) found a decrease in Medicare Part A (plus hospice) spending with $\$ 581$ per quarter in GH homes compared to $\$ 71$ in non-GH homes. Yoon et al. (2015) measured the impact of the GH model on depressive symptoms. The authors reported an increase in depressive symptoms among GH residents compared to traditional NHs (Yoon et al, 2015). Yoon et al. (2016) analyzed the effect of the GH model on activities of daily living (ADL). Yoon et al. (2016) found a decrease in the function of ADL in both NHs (i.e. GH homes and traditional NHs); however, the results were not statistically significant.

Afendulis et al. (2016) found a decrease in bedfast residents (15.8 percent), residents with catheters (45 percent), and residents with low-risk pressure ulcers (38 percent). However, the study did not find a significant impact on incontinence (low risk), restraints, pressure ulcers (high risk), pain, and urinary tract infections (Afendulis et al., 2016). Kane et al. (2007) also found an improvement in residents with little or no activity, residents with late-loss ADLs, and residents with depression symptoms in GH homes. However, there was an increase in incontinence prevalence in $\mathrm{GH}$ home residents compared to traditional NH residents (Kane et al., 2007). The study also reported a higher quality of life in seven out of 11 domains, including privacy, dignity, meaningful activity, relationship, autonomy, food enjoyment, and individuality among residents living GH homes (Kane et al., 2007). Additionally, GH home residents experienced better emotional health compared to residents in traditional NHs. GH home residents also stated that they are more comfortable living and receiving care at their facility compared to those in traditional NHs (Kane et al., 2007). Wolff et al. (2016) introduced a McGeer criteria to 
report UTI rates at the GH home. Prior to the implementation, the incidence rate of UTIs per 1000 bed-days declined from 2.25 in 2011 to 1.08 in 2012 (Wolff et al., 2016). With the introduction of the McGeer criteria, the UTI rate continued to decrease from 0.289 per 1000 bed-days to 0.145 per 1000 bed-days (Wolff et al., 2016).

\section{GH Elements}

\section{Physical Structure}

Cohen et al. (2016) reported that having a smaller physical structure improved the effectiveness of the staff and the closeness between the staff and the residents because the GH staff could hear residents better (Cohen et al., 2016). Regarding location, GH homes are more often located in "normal" neighborhoods where residents can interact with other people (Cohen et al., 2016). One caveat is that the staff do not reside in the GH home but nearby (mostly in the legacy home) and are more likely to not respond to the residents' needs as much as the residents would want them to (Cohen et al., 2016).

\section{Staffing}

Bowers et al. (2016b) reported two sub-elements of the staffing element: the universal role and consistent assignment. The two sub-elements were associated with an increase in familiarity between the staff and the residents (Bowers et al., 2016b). In other words, the staff in GH homes tend to know their clients well compared to traditional NHs (Bowers et al., 2016b). The two sub-components also tend to benefit physicians and nurse practitioners (Bowers et al., 2016b). The clinical team recognized the advantage of the Shahbaz's presence in the GH homes since the Shahbazim are more likely to have detailed information on the residents (Bowers et al., 2016b). However, GH homes with low hospital 
transfer rates were more likely to experience this advantage compared to the GH homes with high hospital transfer rates (Bowers et al., 2016b).

Three studies analyzed the importance of staff communication in the life of the resident (Bowers \& Nolet, 2014; Bowers et al., 2016b; Horn et al., 2016). Nutrition staff and CNAs communicated more often about resident needs with 40.9 percent in GH homes and 0 percent in traditional NHs (Horn et al., 2016). This increase level of communication among the staff was related to a decrease in falls and hospital transfers (Horn et al., 2016). In GH homes, Shahbazim participated more in meetings than CNAs in traditional NHs (90.9 percent vs. 26.7 percent) (Horn et al., 2016). Bowers et al. (2016b) reported that GH homes where the staff leveraged the resources to communicate more effectively observed a low hospital transfer rate. The opposite was also true, with a high hospital transfer rate when the staff in GH homes failed to take advantage of the resources in communicating effectively (Bowers et al., 2016b).

\section{GH Model - Organizational Theory}

The third objective was to identify the use of organizational theory to empirically analyze the GH model. While no studies applied an organizational theory to measure the impact of the model on quality of care, some studies used a conceptual framework and others developed a conceptual framework from their work. Horn et al. (2016) and Yoon et al. (2016) utilized the Donabedian's conceptual framework to analyze the impact of the GH model on quality of care. Two studies created a conceptual framework from their findings (Bowers et al., 2016a; Bowers et al., 2016b). 


\section{DISCUSSION}

Based on our results, the GH model overall and its impact on quality of care were well analyzed in the literature. However, in regard to the GH elements and their impact on quality of care, there was a scarcity of empirical evidence. The authors assessed the impact of the GH model overall on quality of care with these following outcomes: preventable hospitalizations readmissions (Afendulis et al., 2016), Medicare part A spending (Grabowski et al., 2016), psychological symptoms (Yoon et al., 2015), and general quality measures (Afendulis et al., 2016; Kane et al., 2007; Yoon et al., 2016).

The GH elements were found to be consistent with some domains of the culture change including physical environment, resident-centeredness, staffing, and social engagement. The physical structure in GH model as well as in culture change models was found to be different from the one in traditional NHs. For example, the removal of the nurse station and re-arrangement of the kitchen to promote a home-like environment was not only observed in GH homes but also in other culture change NHs (Kane et al., 2007; Tyler \& Lepore, 2017). This type of arrangement provided a welcoming environment not only for the residents but also for their families and staff. Additionally, the change in physical structure was found to positively impact the health of the residents. In the culture change literature, the modification of the physical environment provided comfort to individuals suffering from psychological conditions including Alzheimer's disease and dementia (Day, Carreon, \& Stump, 2000; Wood, Harris, Snider, \& Patchel, 2005), and provided a behavioral change among residents in NHs (Tyler \& Lepore, 2017).

In regard to staffing, staff empowerment was consistent in the GH literature as well as the culture change literature. An improvement in staff empowerment was found to be 
associated with an increase in staff retention and a decrease in staff turnover (Berridge, Tyler, \& Miller, 2018). The opposite was also true in that a decrease in staff empowerment was reported to be associated with a decrease in staff retention and an increase in staff turnover (Berridge et al., 2018). Even though culture change tended to be associated with a positive impact, it also created some unintended consequences related to staff empowerment.

One study reported that some employees were hesitant to assign tasks to other employees while others did not feel responsible to accomplish some tasks (Roberts \& Pulay, 2018). The same study stressed the importance of culture change practices especially in relation to staff empowerment (Roberts \& Pulay, 2018). A staff member from a traditional NH had the intention to incorporate more home-like practices so that residents could be involved in activities, especially cooking activities; however, the employee could not find the courage to address the situation in meetings (Roberts \& Pulay, 2018). This situation created a barrier to staff empowerment in this traditional NH and also shows that changes in organizations can be difficult to incorporate particularly when management is not cooperating. Staff empowerment is important not only for GH homes but also for culture change NHs.

Resident-centeredness plays an important role in the GH model but also in the culture change literature. Roberts and Pulay (2018) reported the experience of a staff who transitioned from a traditional $\mathrm{NH}$ to a culture change $\mathrm{NH}$. In the traditional $\mathrm{NH}$, the staff was obligated to wake up a resident against their will; however, in the culture change $\mathrm{NH}$, residents provided their own wake-up time (Roberts \& Pulay, 2018). Additionally, one 
study reported that resident's ability to control their lives influenced their health (WhiteChu, Graves, Godfrey, Bonner, \& Sloane, 2009).

Some studies found that the presence of the culture change model might be associated with an improvement in social engagement and an increase in falls (Bourgeois et al., 2015; Fritsch et al., 2009; Hermer et al., 2017; Shura et al., 2011). Li and Porock (2014) found that residents in an Eden Alternative NH were less likely to experience loneliness. Residents living in a culture change NH are more likely to engage with the staff in the home (Bourgeois et al., 2015; Fritsch et al., 2009; Hermer et al., 2017; Shura et al., 2011).

\section{CONCEPTUAL FRAMEWORK}

Since there was a scarcity of studies that examined the impact of the GH elements on quality of care using organizational theories, a conceptual framework rooted in the findings and based on the Donabedian's model was developed to illustrate the possible impact of the GH elements (See Figure 2). The Donabedian's model includes three constructs: the structure, process, and outcome components. Structure is defined as "the availability, organization, and financing of healthcare programs; the characteristics of the populations to be served by them' and the physical, social, and economic environments to which they are exposed" (Aday, Begley, Lairson, \& Balkrishan, 2004). Based on that definition, we chose to include the five elements of the GH model (physical structure, staff, residentcenteredness, engagement, and family involvement). In other words, the presence of the five elements is considered as the planning or organization of the healthcare program in this case the GH model. 
Process is defined as "the transactions between patients and providers in the course of actual care delivery, as well as the environmental and behavioral transactions exacerbating health risks" (Aday et al., 2004). Thus, the process component in the context of the GH model includes the activities and participation of the residents and the staff (Cohen et al., 2016). Outcome is defined as "the consequences of policies for the health and well-being of patients and the public" (Aday et al., 2004). In our results, we observed an increase in family satisfaction after the implementation of the GH model, a sense of closeness, and staff efficacy (Lum et al., 2008) (See Figure 2).

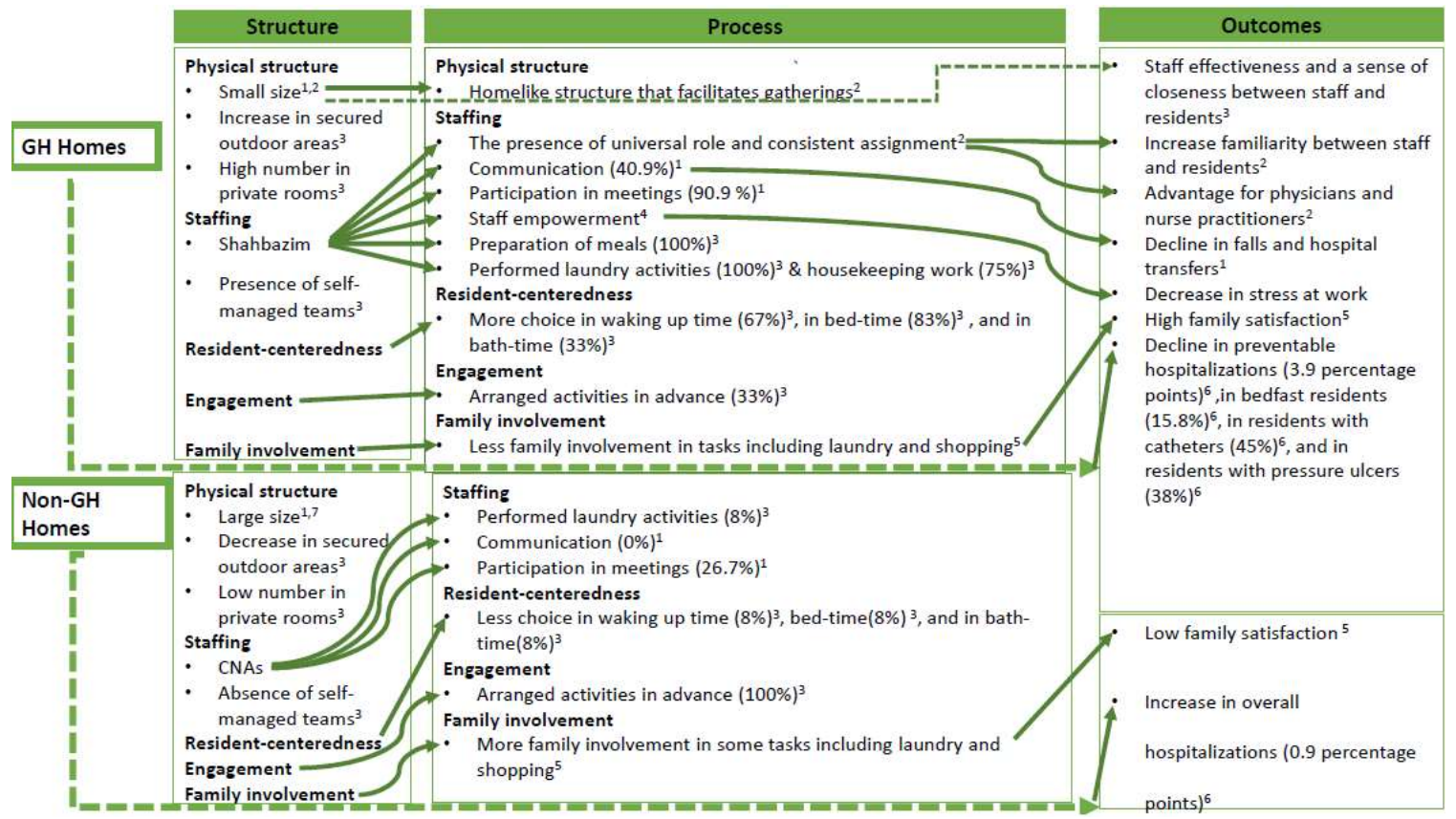

Conceptual Framework: GH Elements and their Impact

Figure 2. Conceptual Framework: GH Elements and their Impact

Footnotes: 1. Horn et al. (2016); 2. Bowers et al. (2016b); 3. Cohen et al. (2016); 4. Loe and Moore (2012); 5. Lum et al. (2008); 6. Afendulis et al. (2016) ; 7. Grabowski et al. (2014). 
Figure 3 provides a simplified conceptual framework which was derived from figure 2. Figure 3 illustrates a relationship between the three components of the Donabedian's model and the implementation of the GH elements (See Figure 3). Based on the structure definition, a GH home should provide a GH design or plan where the presence of a small structure, the Shahbazim, resident-centeredness, engagement, and family involvement is outlined. Thus, the adoption status of the GH model within the structure component represents whether the NH has fully adopted, partially adopted, or did not adopt the GH elements. Next, the process construct was merged with the utilization of the GH elements with three cases possible (i.e. high, low, or no utilization). Last, the outcome construct represented the quality of care based on five scenarios including highest quality of care, high quality of care, low quality of care, lowest quality of care, and no improvement on quality of care.

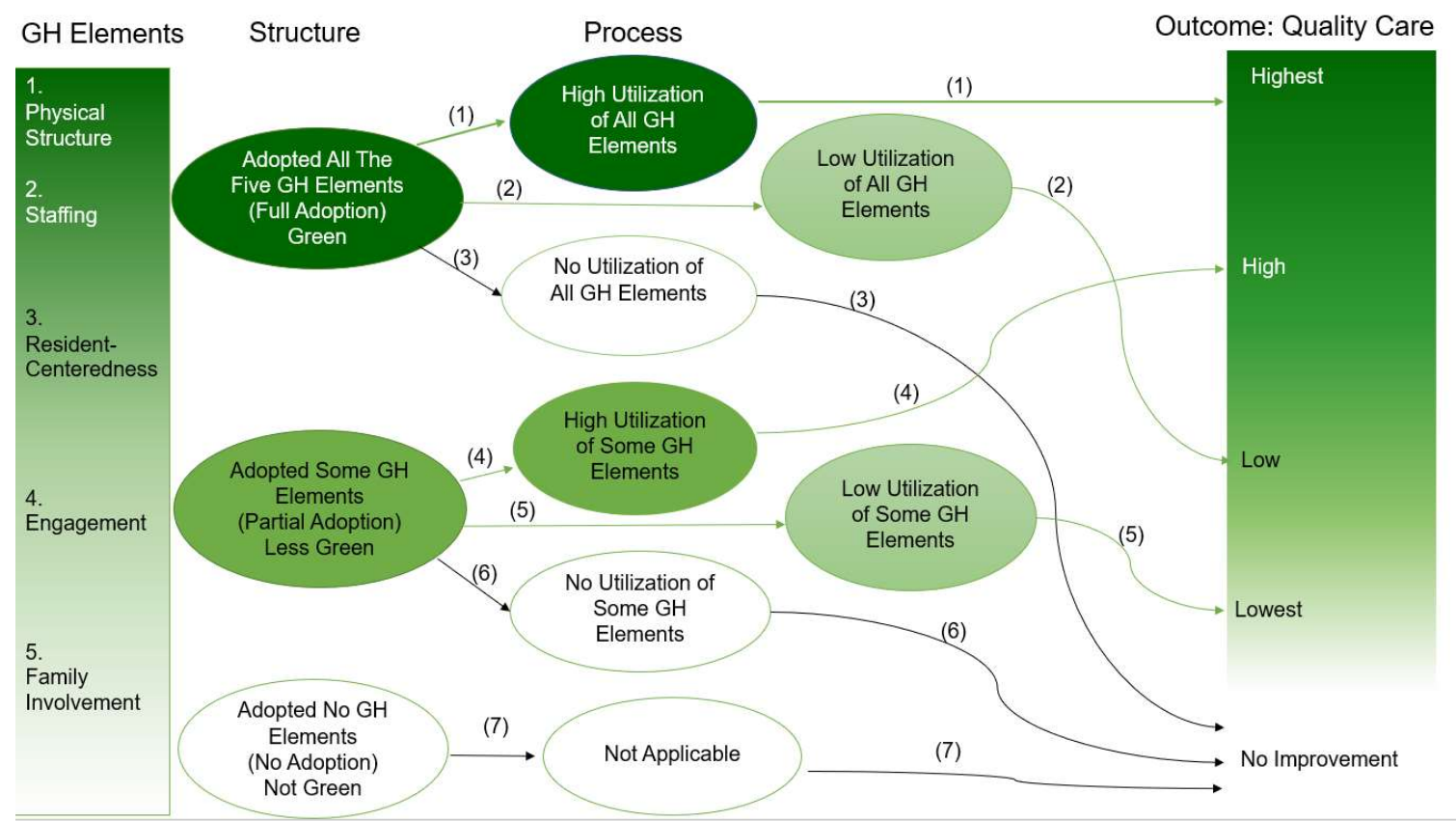

Conceptual Framework: GH Elements and Their Impact on Quality of Care (Simplified Version) 
Figure 3. Conceptual Framework: GH Elements and Their Impact on Quality of Care (Simplified Version). Description for Figure 3:

The color green was chosen because of the GH model. The dark green corresponds to the full adoption, high utilization, and the highest quality of care. The bold green is associated with partial adoption, high utilization, and high quality of care. The light green corresponds to low utilization and low quality of care. The lighter green is associated with lowest quality of care. The color white shows the absence of the GH model and hence the utilization of the model, and the lack of improvement on quality of care. The order of the elements was chosen based on the amount of results that was present in the literature about each GH element. The arrows show the paths for each relation $(S-P-O)$. For example, the NHs that adopted all the GH elements and fully utilized them are more likely to have the highest quality of care. The arrows in that instance showed path (1).

We developed seven scenarios that illustrate the relationship that exists between the GH elements and the three components of the Donabedian's model. However, before explaining this relationship, we consulted the literature on culture change that used a similar approach. Researchers identified four types of NHs which implemented culture change including complete adopters, partially adopters, strivers, and traditional NHs (Doty et al., 2008; Miller et al., 2013; Sterns, Miller, \& Allen, 2010). Complete adopters implemented the culture change model in whole (Doty et al., 2008; Miller et al., 2013; Sterns et al., 2010). Partially adopters are NHs that fully adopted certain practices of the model (Miller et al., 2013). Next, strivers are NHs that partially changed their NHs (Doty et al., 2008; Miller et al., 2013). Lastly, traditional NHs are defined as NHs that did not 
implement the model, but sometimes, conversations are being held about the introduction of the model (Doty et al., 2008; Miller et al., 2013).

Therefore, the seven scenarios were:

1. A NH that claimed to have fully adopted the $\mathrm{GH}$ model and utilized all the GH elements is more likely to achieve the highest quality of care.

2. A NH that claimed to have fully adopted the GH model and utilized some of the GH elements, is more likely to provide a low quality of care.

3. A NH that claimed to have fully adopted the GH model and did not utilize the GH elements, is more likely to not improve quality of care.

4. A NH that claimed to have partially adopted the GH model and yet utilized all the GH elements, is more likely to achieve high quality of care.

5. A NH that claimed to have partially adopted the GH model and utilized some of the GH elements, is more likely to provide the lowest quality of care

6. A NH that claimed to have partially adopted the GH model and did not utilize the GH elements, is more likely to not improve quality of care.

7. A NH that did not implement the GH model is more likely to not improve quality of care.

The literature showed that most NHs that claimed to have fully adopted the GH model and poorly managed them, were associated with a negative impact. For example, the presence of outdoor space helps with residents to enjoy fresh air (Cohen et al., 2016). However, some studies found that the outdoor space was not used because they did not want the residents to escape. Bowers et al. (2016a) provided a case where a resident wandered off from a $\mathrm{NH}$ with no supervision, then the facility received a fine once the 
management reported the incident to the authorities. As a result, restrictions from the state were applied (Bowers et al., 2016a). The study reported that the situation became worse than the traditional management (Bowers et al., 2016a). In this case, even though, outdoor space was present, it was not utilized according to the GH model design. This demonstrates the presence of the GH elements and the low utilization which might lead to a decline in quality of care, especially since fresh air helps with psychological symptoms.

\section{LIMITATIONS}

Few studies analyzed the GH model and its impact on the quality of care. The culture change literature experienced a similar problem with little empirical evidence. This problem is mainly attributed to the size of the sample. Various studies reported that the sample size was small. Therefore, a threat to external validity exists where the findings cannot be generalized to other NHs. Another limitation was observed with the mix of findings. Some studies reported positive outcomes associated with the GH model while others found negative results related to the model, which may be attributed to study year. For example, overtime, the impact of the GH model on social engagement changed from being negative to positive. Thus, further research is necessary since implementation of the GH model is increasing. 


\section{POLICY AND PRACTICE IMPLICATIONS}

Future research should identify the effect of each GH element and its outcomes related to quality of care. Zimmerman et al. (2016) agreed that a need to know which GH element influences quality of care among residents is necessary. Additionally, identifying which $\mathrm{GH}$ element positively impacts quality of care will help with saving time and financial resources. Future NHs contemplating to adopt the GH model will more likely focus on implementing the $\mathrm{GH}$ elements that are effective. Thus, NH leadership, culture change experts, policy makers, researchers, and other stakeholders may want to invest in the GH elements that have been proven beneficial to the residents and the staff.

In regard to culture change, a policy has already been implemented that encourages NHs to adopt culture change practices. CMS has developed a way for NHs to be reimbursed through the pay for performance (P4P) programs to incentivize NHs that adopted culture change (Grabowski et al., 2014a; Doll et al., 2017; Roberts et al., 2018).

Prior to the culture change movement, traditional NHs were considered as the final step before death; however, with the culture change movement, the situation is shifting from a worse to a much better environment not only for the residents but also for employees. However, even with an innovative movement like culture change, challenges still exist, but every innovation takes time to take roots and flourish. 


\section{CHAPTER 3: AIM 2}

The Green House Nursing Home Model: The Deficiency Citations and the Model

\section{Elements}

Deficiency citations are given to nursing homes (NHs) that did not meet the Centers for Medicare and Medicaid (CMS) requirements (as cited in Castle, Wagner, Ferguson, \& Handler, 2011a). According to the Department of Health and Human Services (DHHS) (2004) (as cited in Castle, 2011b), a deficiency citation is defined as "a finding that a nursing home failed to meet one or more federal or state requirements." According to the 2019 DHHS report, 94 percent of deficiencies were less severe (labels D, E, F) compared to 6 percent of deficiencies that were more severe (labels G-L, or with F with a poor quality). Based on the DHHS report, an increase in the number of deficiencies was observed from 2013 to 2016, then a decrease in the number of deficiencies was observed in 2017 (DHHS, 2019).

The federal government is the primary payer of $\mathrm{NH}$ care through Medicare and Medicaid and manages quality of care through those two programs (Castle, Engberg, \& Men, 2007). NHs that receive Medicaid or Medicare reimbursement are required to meet the minimum standards by going through a certification process (Castle et al., 2007). In addition to receiving a deficiency citation, a NH which does not align with the federal requirements, two possible scenarios can occur. 
Either the NH can be fined up to $\$ 10,000$ per day (Castle, 2011b) or, the facility will no longer receive reimbursements from the Medicaid or Medicare programs (Castle, 2011b). A deficiency citation is identified by the scope and severity of the issue. Twelve categories exist from A to L, which assess the gravity of the deficiency (Castle, et al., 2011a; Temkin et al., 2010). The A category is defined as the least severe while L is determined as the most severe (Castle et al., 2011a; Temkin et al., 2010). Severity is defined as the level of harm experienced by the resident while scope is the total number of residents who suffered such abuse with isolated cases, a pattern of cases, and widespread cases (Castle et al., 2011a; Grabowski et al., 2016). Figure 4 provides more information on the scope and severity of the deficiency citations.

In the NH literature, deficiency citations are mostly used as indicators of quality of care indicators. A NH with a high number of deficiency citations is more likely to be associated with poor quality (Temkin et al., 2010). Once a NH receives a high number of deficiency citations, it is threatened to immediate closure if no action is taken to correct the problem(s) issued by the federal government (Castle \& Ferguson, 2010). However, research has demonstrated that NHs with an increasing number of deficiency citations are more likely to correct the problem(s) (Mukamel et al., 2007).

In addition to deficiency citations, the culture change movement is another solution that was created to improve the quality of care in NHs. The objective of the culture change movement is to deinstitutionalize NHs with a focus on the residents (Miller et al., 2013). The culture change movement incorporates various types including the GH model, the Eden Alternative, the Pioneer Network, the Meadowland Hills Program, Wellspring, and Planetree (Cohen et al., 2016; Ragsdale \& McDougall, 2008). For the purpose of this 
research, we will focus on the GH model with an effort to answer this following research question: "Which GH model elements have an effect on the deficiency citations and what are these effects?" Our research objective is to analyze the impact of the GH model on the deficiency of citations.

\begin{tabular}{|l|l|l|l|}
\hline & \multicolumn{3}{c|}{ Scope } \\
\hline \multicolumn{1}{|c|}{ Severity } & Isolated & Pattern & Widespread \\
\hline $\begin{array}{l}\text { Immediate Jeopardy: immediate jeopardy to } \\
\text { resident health or safety }\end{array}$ & $\mathrm{J}^{* *}$ & $\mathrm{~K}^{* *}$ & $\mathrm{~L}^{* *}$ \\
\hline Actual Harm: that is not immediate jeopardy & $\mathrm{G}$ & $\mathrm{H}^{* *}$ & $\mathrm{I}^{* *}$ \\
\hline $\begin{array}{l}\text { Potential for Actual Harm: no actual harm } \\
\text { with potential for more than minimal harm that } \\
\text { is not immediate jeopardy }\end{array}$ & $\mathrm{D}$ & $\mathrm{E}$ & $\mathrm{F}$ \\
\hline $\begin{array}{l}\text { Minimal Harm: no actual harm with potential } \\
\text { for minimal harm }\end{array}$ & $\mathrm{A}$ & $\mathrm{B}$ & $\mathrm{C}$ \\
\hline
\end{tabular}

Deficiency Citations: Scope and Severity. With ** Substandard Quality of Care: if tag between 221 and 258 or tag between 309 and 333 and scope is F or $\mathrm{H}<->$ L. CMS Manual

Figure 4. Deficiency Citations: Scope and Severity

\section{BACKGROUND}

Developed out of the Omnibus Reconciliation Act in 1987 (OBRA-87), the culture change movement sought to deinstitutionalize NHs (Bowers \& Jacobson, 2016; Cohen et al., 2016). Prior to the law in 1987 , NHs were considered as facilities with a hospital-like environment (Yoon et al., 2015). However, a group of consumers raised awareness on the problems faced by the residents (Koren, 2010). As a response, the Institute of Medicine (IOM) altered the regulatory measures so that $\mathrm{NH}$ residents can obtain better quality of care (Koren, 2010). 
The OBRA-87 act aimed at decreasing the likelihood of poor quality of care observed in NHs (Cohen et al., 2016). The resident assessment instrument and minimum data set (MDS) were created to report quality measures of NHs to the public (Cohen et al., 2016). Out of the OBRA-87 policy, the culture change movement was also created (Cohen et al., 2016). Various types of culture change movement exist including the Eden Alternative, the Wellspring, the Planetree, and the GH model (Cohen et al., 2016; Miller, Mor, \& Burgess, 2016). The main objective of the culture change movement is to deinstitutionalize the NHs and to provide resident-centeredness care services (Koren, 2010). For the purpose of this research, we will focus on the GH model because it is the latest model of the culture change movement.

\section{Green House Model Description}

The GH model, created in 2003, has an objective of deinstitutionalizing the long-term care sector and has a target on person-centered care (Rabig et al., 2006). A GH model includes a small home which provides a private room, bathroom, and a common area for dining purposes to its residents who can total up to 12 (Afendulis et al., 2016; Cohen et al., 2016; Grabowski et al., 2016; Rabig et al., 2006; Zimmerman et al., 2016). GH homes have augmented from four homes in 2003 to 174 homes in 2015 (Lum et al., 2008; Zimmerman et al., 2016). Currently, there are $237 \mathrm{GH}$ homes that are in operation and $60 \mathrm{GH}$ homes are in development in 31 states ("Find a home", 2020). Some GH homes share a license with a traditional NH (called legacy homes), while others have their own license (Afendulis et al., 2016).

A GH home uses various types of staffing that differs from that used in traditional NHs. GH staff often include a Shahbaz, a Guide, a Sage, and other non-clinical staff 
(Ragsdale \& McDougall, 2008). The Shahbaz is the equivalent of a certified nursing assistant (CNA) in traditional NHs (Afendulis et al., 2016). However, the Shahbazim (Plural of Shahbaz) have more tasks than do traditional CNAs (Brown et al., 2016), and are considered "universal caregivers" (Cohen et al., 2016). Shahbazim are responsible for preparing meals, doing laundry, housekeeping, and supporting residents' personal care and overall quality of life (Brown et al., 2016; Cohen et al., 2016; Rabig, et al., 2006). Shahbazim have a manager called a Guide who oversees the Shahbazim work (Rabig et al., 2006). A Sage is a community member carefully chosen by the GH Guide and staff, who volunteers to provide advice to the Shahbazim and support them in regard to decisionmaking and problem-solving duties (Ragsdale \& McDougall, 2008). A group of social workers, dieticians, nurses, and activity personnel stays near to the GH homes so they can provide services to the residents (Rabig et al., 2006; Ragsdale \& McDougall, 2008). Thus, person-centeredness, small homes, room privacy, and a special team of employees are some of the key elements of the GH model.

In the literature, there is a scarce evidence of the GH impact on the deficiency citations. Grabowski et al. (2014a) did not find any significant difference between the GH facilities and the non-GH facilities. However, when the authors analyzed the effect of the culture change models on the deficiency citations, a decrease in the number of deficiency citations was found in NHs that implemented the culture change compared to those that did not (Grabowski et al., 2014a). Therefore, empirical evidence that demonstrates the effect of the GH model on the deficiency citations is needed. In addition, a gap exists in the literature in terms of examining how specific elements of the GH model impact quality of care. Zimmerman and Cohen (2010) reported in their study that a consensus has been 
reached that the six key elements of the GH model include physical structure, staffing, dining, resident-case mix, resident-centeredness, and engagement (Zimmerman \& Cohen, 2010). Cohen et al. (2016) and Zimmerman et al. (2016) stressed the importance of identifying which element has an impact on quality of care. Therefore, it is important to analyze which GH components influence quality of care. For the purpose of our research, we focused on the physical structure and staffing since the two elements are available in our dataset.

\section{CONCEPTUAL FRAMEWORK: DONABEDIAN MODEL}

In the NH literature, the Donabedian model is often applied to accomplish various projects (Horn et al., 2016; Yoon et al., 2016). For this research paper, we used the Donabedian model to describe the impact of the GH model on the quality of care. The Donabedian model includes three components: structure, process and outcome of the healthcare system (Aday et al., 2004). The NH literature often demonstrates through the Donabedian model that an effective structure, a facility will provide great care process which leads to improved outcome (Yoon et al., 2016).

Structure is defined as "the availability, organization, and financing of healthcare programs; the characteristics of the populations to be served by them; and the physical, social, and economic environments to which they are exposed." (Aday et al., 2004). Based on this definition, the availability of the $\mathrm{GH}$ model within the $\mathrm{NH}$ is incorporated in this construct. In other words, the five GH elements including the physical structure, the staff, the resident-centeredness, the engagement, and the family involvement are included in the structure component. In this study, only two elements were available in the dataset which were the physical structure and the staffing components. 
According to Aday (2004), process is defined as "the transactions between patients and providers in the course of actual care delivery, as well as the environmental and behavioral transactions exacerbating health risks." In our case, the prevalence of restraints was used as a process variable as cited in the literature (Castle \& Ferguson, 2010). Based on the process definition, we understand that there is a relationship between the patients and providers and in our case, a decrease in the use of restraints should improve the quality of care (Konetzka et al., 2014). Thus, the GH model should promote the decrease, even the elimination of restraints among residents. Therefore, the restraint variable is an indicator of poor quality of care (Konetzka et al., 2014) since its presence leads to cognitive issues and other diseases.

Lastly, outcome is defined as "the consequences of policies for the health and wellbeing of patients and the public" (Aday et al., 2004). In our case, the outcome variable is the number of deficiency citations which has been used as a measure of quality of care in the literature (Castle \& Myers, 2006). Thus, with an effective structure, a facility will be able to provide better health care process which will lead to improved outcomes in this case the quality of care (Yoon et al., 2016) (View Figure 5).

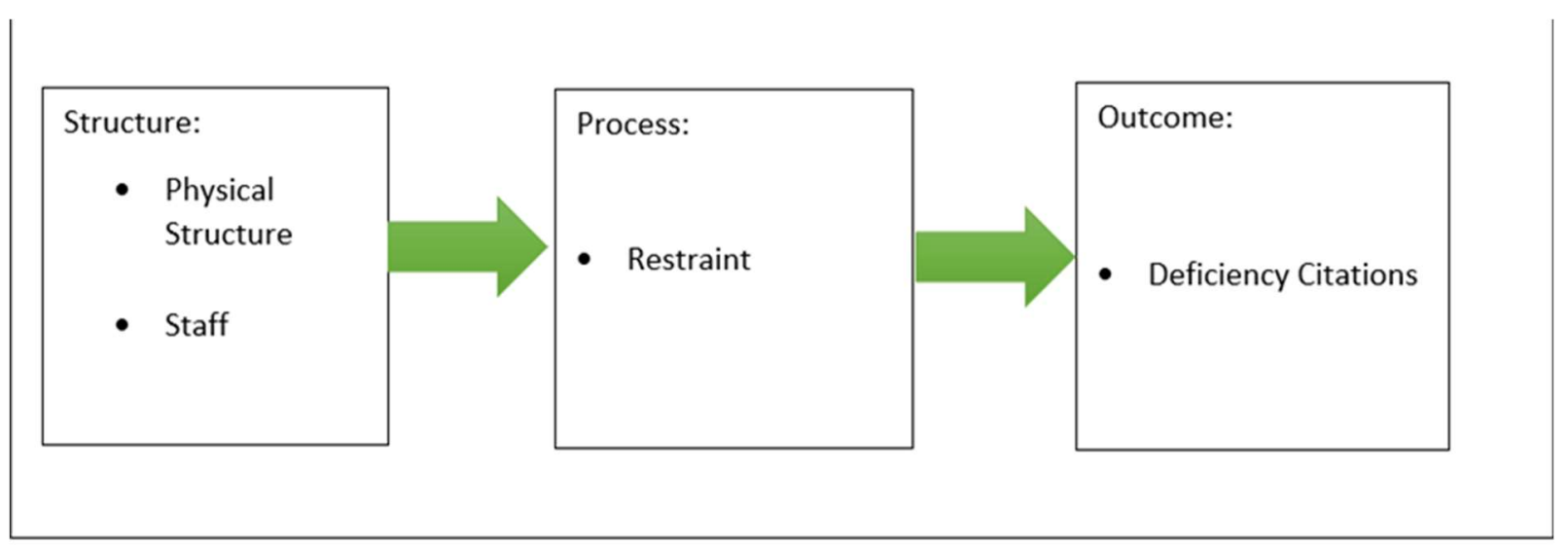

Conceptual Framework: Deficiency Citations

Figure 5. Conceptual Framework 


\section{METHODS}

The Long-Term Care Focus dataset (LTCFocus) located at the Brown University Center for Gerontology website from 2000 to 2017 was merged with the Nursing Home Compare (NHC) dataset which is publicly available to conduct this research project. The LTCFocus combines various datasets including the Online Survey Certification and Reporting/Certification and Survey Provider Enhanced Reporting (OSCAR/CASPER), Minimum Data Set (MDS), the Area Resource File (ARF), the Residential History File (RHF), NHC, and the State Policy Data (LTCFocus, 2017). The NHC dataset was separately obtained because it provided the deficiency citations variable which was not available in the LTCFocus dataset. Before merging the two datasets, we collapsed the NHC using the scope and severity variable and the first survey. In the process, we created new variables that described the range of harm including minimal harm, potential for harm, actual harm, and immediate jeopardy harm. Then, we used the NH identifier to merge the LTCFocus and the NHC datasets. Years 2000 to 2017 were appended in one final dataset. The Green House Project provided a list of NHs that implemented the model and the time for implementation ("Find a home", 2020).

\section{Exclusion Criteria}

We excluded pediatric, veteran, and hospital-based facilities (Sharkey et al., 2011). Pediatric facilities were identified by noting NHs where the average age in the $\mathrm{NH}$ was equal or less than 21 years old and by noting NHs with names that contained children/kids/pediatrics. Veteran-based facilities were identified by observing NH names that included veterans or vets. Facilities were directly identified in the LTCFocus as hospital-based facilities, which were excluded due to concerns about non-comparability 
with other NHs (For example, hospital-based facilities can be much larger - up to 1,098 beds - than non-hospital NHs).

Also, for non-comparability reasons, assisted living facilities were not included in the analysis. Because the statistical methods relied on matching GH NHs to non-GH NHs by state, states where there were no GH facilities were necessarily excluded from the dataset. Alabama, Arkansas, California, Colorado, Florida, Kansas, Massachusetts, Michigan, Mississippi, Montana, New Jersey, Ohio, Oregon, Pennsylvania, Rhode Island, Tennessee, and Virginia were included in the final dataset. In order to directly examine the effect of adopting the GH model, we excluded eleven GH facilities for which data was not available for the entirety of 2000-2017 (which eliminated GH facilities that were created from new using the GH model rather than converting to the GH model).

\section{Treated/Untreated Facilities}

As discussed above, we matched GH (treated) facilities to non-GH (untreated) facilities before conducting our analyses. Matching was done using propensity scores calculated using the p-score command in Stata. With that method, a p-score for each facility (specifically, the propensity of being a GH home in or before 2017) was calculated using data from the baseline year in the data (Grabowski et al., 2014b) and the multi-facility and for-profit variables as predictors. Per Grabowski et al. (2014b), we sought a ratio of 10 matching non-GH NHs for every GH NH in the analysis, with matches being constrained to be in the same state as the GH home (to control for unobserved effects of state policies). In instances where more than 10 non-GH facilities were tied with the same p-score, a random sampling method was used to choose the 10 control facilities. In the one instance 
in which there were fewer than 10 available matches, we used all four available facilities as controls.

Eleven treated facilities were not present before their implementation and were therefore removed since observations prior to implementation were nonexistent. Another caveat was that since one GH facility did not have data in one year (2002), we had to interpolate the data by creating an average from the prior year (2001) and the subsequent year (2003) for each variable in the dataset.

The resulting dataset for the analyses contained a total of 280 facilities (26 GH and 254 non-GH) across 18 years. The 26 treated facilities implemented the GH model at different points in time with 1 in 2004, 1 in 2006, 5 in 2007, 1 in 2008, 2 in 2009, 2 in 2010, 2 in 2011, 3 in 2012, 1 in 2013, 5 in 2016, and 3 in 2017.

\section{Dependent and Independent Variables}

\section{Dependent Variable}

\section{Deficiency Citations}

The outcome variable is the total number of deficiency citations which was provided by the NHC dataset. The deficiency citations has been used in the literature for analysis purposes (Temkin et al., 2010; Grabowski et al., 2014a; Grabowski et al., 2014b). The deficiency citations were also classified into four groups. Figure 4 presents the four categories of the deficiency citations by scope and severity. First, the minimal harm includes a scope of isolated (A), pattern (B), and widespread (C) cases (Temkin et al., 2010). Within this category, the range of harm expands from no harm to possibility for minimal harm (Temkin et al., 2010). Secondly, the potential for actual harm is designated with a scope of isolated (D), pattern (E), and widespread (F) cases (Temkin et al., 2010). 
Within this category, the facility increases its probability to more than minimal harm which does not make the residents in immediate risk (Temkin et al., 2010). Next, the actual harm incorporates a scope of isolated $(\mathrm{G})$, pattern $(\mathrm{H})$, and widespread (I) cases (Temkin et al., 2010). The last category is labeled as immediate jeopardy with a scope of isolated (J), pattern (K), and widespread (L) cases (Temkin et al., 2010). In this instance, the facility receives a deficiency citation for putting the residents' health or safety in immediate jeopardy (Temkin et al., 2010).

\section{Independent Variables}

The primary independent variables were chosen based on a combination of the GH elements available in the dataset and the Donabedian's model (Structure-ProcessOutcome). The GH elements are the physical structure, the staff, the resident-centeredness, engagement, and family involvement (Zimmerman \& Cohen, 2010).

The GH elements that are embedded in the structure component are physical structure and staff. The physical structure and staff are the only elements available in the LTCFocus dataset. Based on the literature, the physical structure was defined as the size of the beds, i.e. the number of beds (Zimmerman \& Cohen, 2010; Afendulis et al., 2016; Grabowski et al., 2016). The staff GH element includes the RN hours per day, the LPN hours per day, and the CNA hours per day. For the GH model, the staff are Shahbazim which are an equivalent of the CNA in GH facilities (Afendulis et al., 2016). These variables are embedded within the structure component of the Donabedian's structure. The percentage of restraint was used as the process variable (Castle \& Ferguson, 2010). 


\section{Covariates}

In addition to the main variables, age, gender, race, and payers will be controlled for in the analysis. Age will be defined as those who are 65 years and older. The gender variable will be considered as a categorical variable with female or male as the coded variable. The dataset provides race/ethnicity variables including White, Black, and Hispanic. The payer variable recognizes whether the resident was supported by Medicaid or Medicare. These variables were aggregated to the facility, county, and state levels.

\section{Fixed Effect Variables}

Two fixed effect variables were created for analysis purposes. A facility fixed-effect variable and a time fixed effect variable were created for the difference-in-difference method. An interaction term was also created where the treatment was multiplied with the time fixed-effect variable.

\section{Missing Values}

To manage the missing values, six scenarios were created for robustness purposes. The LTCFocus dataset has observations with low number of events (LNEs) values (Needleman, Kim, Jung, \& Unruh, 2018). In other words, with a low number of observations for a given facility, might risk to reveal the identity of the NH residents (Needleman et al., 2018). Thus, in addition to the normal missing values, a number of LNE values was present in the dataset. To manage this issue, we created six scenarios where we dealt with LNE values in the first three scenarios and managed with LNE values and other type of missing values in the last three scenarios. Below is a description of each scenario. For the purpose of this research, we focused on the sixth scenario since it dealt with the LNE values and the other type of missing values. Additionally, in this case, observations were not removed and the 
summary statistics made sense. Lastly, results were consistent across all scenarios (View Appendix).

\section{Method for analysis}

A difference-in-difference method was used to conduct the analysis with the help of Stata14 as the software. A poisson regression model was performed. After this, two new variables were created to measure the change in the pre-and post-periods. The equation that was used:

$$
\mathrm{Y}_{\mathrm{it}}=\beta_{0}+\beta_{1} * \mathrm{~T}_{\mathrm{i}}+\beta_{2} * \text { Year }_{\mathrm{t}}+\beta_{3} *\left(\mathrm{~T}_{\mathrm{i}} * \text { Year }_{\mathrm{t}}\right)+\beta_{4} * \mathrm{X}_{\mathrm{it}}+\mu \mathrm{GH}_{\mathrm{i}}+\Omega \mathrm{TI}_{\mathrm{t}}+\mathrm{e}_{\mathrm{it}}
$$

Where i represents the nursing home facility at time $t, Y$ represents the dependent variable: the total number of deficiency citations. Furthermore, $\mathrm{T}$ indicates the $\mathrm{GH}$ adoption variable, Year represents the time variable, $\mathrm{X}$ indicates the covariates (the number of beds, the RN hours per day, the LPN hours per day, the CNA hours per day, restraint, age, sex, race, payer).

Two fixed effect variables were also used including the nursing home fixed effect variable, GH and the time fixed effect variable, TI. For the facility fixed effect variable, a dichotomy variable was generated where 1 represented the adoption of the GH model and 0 indicated the non-adoption of the GH model. Regarding the time fixed effect variable, a dummy variable was created for each year where 1 represented observations present in the year of interest and 0 indicated observations present in other years. Interaction terms were

used by multiplying the treatment variable with the time variable for the difference-indifference method totaling to a number of 18 interaction terms for each year. 


\section{RESULTS}

Table 2 presents the findings by structure, process of care, and outcome for the GH facilities compared to the non-GH facilities. The number of beds were higher in GH facilities (mean=131.66, $\mathrm{SD}=5.56$ ) compared to non-GH facilities (mean=119.95, $\mathrm{SD}=1.13)$. In terms of staffing, $\mathrm{GH}$ facilities had more staff hours comparing to non-GH facilities with $\mathrm{RN}$ hours per day (mean $=0.43, \mathrm{SD}=0.02$ ), $\mathrm{LPN}$ hours per day (mean $=0.98$, $\mathrm{SD}=0.02$ ), and CNA hours per day (mean=3.11, $\mathrm{SD}=0.08$ ) in $\mathrm{GH}$ facilities, and $\mathrm{RN}$ hours per day (mean $=0.41, \mathrm{SD}=0.01$ ), $\mathrm{LPN}$ hours per day (mean $=0.82, \mathrm{SD}=0.01$ ), and CNA hours per day (mean $=2.59, \mathrm{SD}=0.02)$ in non-GH facilities.

Regarding the process variable, residents who were restrained were more in non$\mathrm{GH}$ facilities (mean=5.35, $\mathrm{SD}=0.12$ ) compared to $\mathrm{GH}$ facilities (mean=3.02, $\mathrm{SD}=0.50$ ). For the covariates, $\mathrm{GH}$ facilities had more female residents (mean=77.39, $\mathrm{SD}=0.46$ ), White residents(mean $=90.39, \mathrm{SD}=0.97)$, and older residents (mean=85.83, $\mathrm{SD}=0.19)$ comparing to residents in non-GH facilities with female residents(mean=75.05, $\mathrm{SD}=0.14)$, White residents $($ mean $=89.22, \mathrm{SD}=0.24$ ), and older residents (mean=83.60, $\mathrm{SD}=0.34)$. The percentage of residents who paid through Medicaid was less in $\mathrm{GH}$ facilities (mean=50.34, $\mathrm{SD}=1.50$ ) comparing to non-GH facilities (mean $=57.76, \mathrm{SD}=0.34$ ). The percentage of residents who paid through Medicare was slightly more in GH facilities (mean=12.68, $\mathrm{SD}=0.60$ ) compared to non-GH facilities (mean $=11.78, \mathrm{SD}=0.17$ ).

For the outcome variable, we were interested in the total number of deficiency citations and the scope of the deficiency (i.e., minimal harm, potential harm, actual harm, and immediate harm). For the total number of deficiency citations, the GH facilities (mean=4.67, $\mathrm{SD}=0.28$ ) had a lower number of deficiency citations compared to non-GH 
facilities (mean $=5.13, \mathrm{SD}=0.07)$. $\mathrm{GH}$ facilities (mean $=0.27, \mathrm{SD}=0.05$ ) had a lower number of minimal harm compared to non-GH facilities (mean $=0.40, \mathrm{SD}=0.01$ ). $\mathrm{GH}$ facilities (mean $=4.2114, \mathrm{SD}=0.2593$ ) had a lower number of potential harm compared to non-GH facilities (mean=4.4452, $\mathrm{SD}=0.0641)$. $\mathrm{GH}$ facilities (mean $=0.1714, \mathrm{SD}=0.0348)$ had a lower number of actual harm compared to non-GH facilities (mean=0.2265, $\mathrm{SD}=0.0103$ ). GH facilities (mean $=0.0229, \mathrm{SD}=0.0139$ ) had a lower number of immediate harm compared to non-GH facilities (mean $=0.0617, \mathrm{SD}=0.0072$ ).

\section{Table 2.}

\section{$\underline{\text { Descriptive Statistics }}$}

\begin{tabular}{|c|c|c|c|}
\hline Facilities & GH Facilities & Non-GH Facilities & Total \\
\hline \multicolumn{4}{|l|}{ Structure } \\
\hline Total Beds (SD) & $131.6571(5.56)$ & $119.9472(1.13)$ & $120.35(1.1099)$ \\
\hline $\mathrm{RN}(\mathrm{SD})$ & $.425(0.0179)$ & $.412(0.0064)$ & $.4129(0.0062)$ \\
\hline LPN (SD) & $.9752(0.0237)$ & $.8152(0.0103)$ & $.8207(0.0099)$ \\
\hline CNA (SD) & $3.1097(0.0780)$ & $2.5863(0.0166)$ & $2.6045(0.0163)$ \\
\hline \multicolumn{4}{|l|}{ Process } \\
\hline Restrain (SD) & $3.0058(0.5014)$ & $5.3482(0.1181)$ & $5.2668(0.1154)$ \\
\hline \multicolumn{4}{|l|}{ Covariates } \\
\hline Female (SD) & $77.3862(0.4603)$ & $75.0467(0.1410)$ & $75.13(0.1371)$ \\
\hline White (SD) & $90.3899(0.9715)$ & $89.2203(0.2444)$ & $89.2609(0.2384)$ \\
\hline Average age (SD) & $85.8318(0.1945)$ & $83.5997(0.0692)$ & $83.6772(0.0673)$ \\
\hline Medicaid (SD) & $50.3361(1.5022)$ & $57.7610(0.3355)$ & $57.5031(0.3285)$ \\
\hline Medicare (SD) & $12.6796(0.5965)$ & $11.7834(0.1715)$ & $11.8145(0.1668)$ \\
\hline \multicolumn{4}{|l|}{ Outcome } \\
\hline Deficiencies (SD) & $4.6743(0.2810)$ & $5.1320(0.07371)$ & $5.1161(0.0718)$ \\
\hline Minimal harm (SD) & $0.2686(0.0488)$ & $0.3986(0.0127)$ & $0.3940(0.1238)$ \\
\hline Potential harm (SD) & $4.2114(0.2593)$ & $4.4452(0.0641)$ & $4.4371(0.0625)$ \\
\hline Actual harm (SD) & $0.1714(0.0348)$ & $0.2265(0.0103)$ & $0.2246(0.0100)$ \\
\hline Immediate harm (SD) & $0.0229(0.0139)$ & $0.0617(0.0072)$ & $0.0603(0.0069)$ \\
\hline
\end{tabular}

Table 3 shows the associations between the independent variables and the number of deficiency variables among GH facilities compared to non-GH facilities. No association 
was found between the GH facilities and the non-GH facilities when it comes to deficiency citations $(\mathrm{IRR}=0.917866 ; \mathrm{p}$-value $=0.454)$.

\section{Structure Variables}

The number of beds was 0.059 percent higher among GH facilities compared to the number of beds in non-GH facilities ( $I R R=1.000592$; $p$-value $<.001$ ). The number of the $\mathrm{RN}$ hours per day was 37 percent lower in $\mathrm{GH}$ facilities compared to the $\mathrm{RN}$ hours per day in non-GH facilities $(\mathrm{IRR}=0.6306001 ; \mathrm{p}$-value $<.001)$. There was no statistically significant difference to suggest that there was an association between the LPN hours per day and the deficiency citations in GH facilities compared to non-GH facilities (IRR=0.9855432; $\mathrm{p}$ value $=0.254$ ). The number of the CNA hours per day was 7 percent lower in GH facilities compared to the CNA hours per day in non-GH facilities $(\operatorname{IRR}=0.9374117$; $\mathrm{p}$-value $<.001)$.

\section{Process Variable}

The number of restrained residents was 0.5 percent higher in $\mathrm{GH}$ facilities compared to restrained residents in non-GH facilities $(\mathrm{IRR}=1.00551 ; \mathrm{p}$-value $<.001)$.

\section{Covariates}

The number of female residents was one percent lower in GH facilities compared to female residents in non-GH facilities ( $I R R=0.99$, $p$-value $<0.001$ ). Age was not statistically significant $(\mathrm{IRR}=0.998 ; \mathrm{p}$-value $=0.279)$. The number of White residents was one percent lower in $\mathrm{GH}$ facilities compared to White residents in non-GH facilities $(\mathrm{IRR}=0.9972378 ; \mathrm{p}$-value $<.001)$. Residents with Medicaid were more likely to be associated with deficiency citations in $\mathrm{GH}$ facilities compared to non-GH facilities $(\mathrm{IRR}=1.000757 ; \mathrm{p}$-value $=0.05)$. Residents with Medicare were more likely to be associated 
with deficiency citations in GH facilities compared to non-GH facilities (IRR=1.011084; p-value $<0.001)$.

\section{Table 3.}

$\underline{\text { Poisson Regression Model Predicting the Number of Deficiency Citations }}$

\begin{tabular}{|c|c|c|c|c|}
\hline Variable & Incidence Rate & $95 \%$ Confi & Interval & p-value \\
\hline $\begin{array}{l}\text { Green House } \\
\text { facilities }\end{array}$ & 0.917866 & 0.733352 & 1.148805 & 0.454 \\
\hline Number of beds & 1.000592 & 1.000423 & 1.000761 & $<.001$ \\
\hline $\begin{array}{l}\text { RN hours per } \\
\text { day }\end{array}$ & 0.6306001 & 0.599065 & 0.663795 & $<.001$ \\
\hline $\begin{array}{l}\text { LPN hours per } \\
\text { day }\end{array}$ & 0.9855432 & 0.96121 & 1.010492 & 0.254 \\
\hline $\begin{array}{l}\text { CNA hours per } \\
\text { day }\end{array}$ & 0.9374117 & 0.922866 & 0.952186 & $<.001$ \\
\hline \% Restrain & 1.00551 & 1.003987 & 1.007034 & $<.001$ \\
\hline Average age & 0.9977543 & 0.993702 & 1.001823 & 0.279 \\
\hline$\%$ Female & 0.9908291 & 0.98905 & 0.992611 & $<.001$ \\
\hline \% White & 0.9972378 & 0.996213 & 0.998264 & $<.001$ \\
\hline$\%$ Medicaid & 1.000757 & 0.999998 & 1.001516 & 0.051 \\
\hline$\%$ Medicare & 1.011084 & 1.009818 & 1.012351 & $<.001$ \\
\hline Constant & 19.52412 & 2.658697 & 3.284604 & $<.001$ \\
\hline
\end{tabular}

Table 3

\section{DISCUSSION}

There is limited research on the impact of the GH model on deficiency citations. Most prior research explored the impact of the culture change in general and other programs such as the Promoting Excellent Alternatives in Kansas (PEAK 2.0) program and the Pay for 
Performance (P4P) program. Deficiency citations have been used as measures for quality of care in NHs where on the one hand, an increase in deficiency citations is associated with a poor quality of care. On the other hand, a decrease in deficiency citations is considered as an improvement in quality of care in NHs.

Our results though not significant suggest that the GH model may have a positive impact on the quality of care. In other words, GH facilities had a decrease in deficiency citations compared to non-GH facilities (IRR: 0.92; p-value: 0.454) (See Table 2). Table 2 presents the descriptive statistics between the GH facilities and non-GH facilities. In terms of the deficiency citations, the $\mathrm{GH}$ facilities (mean $=4.67 ; \mathrm{SD}=0.28$ ) had fewer deficiency citations compared to non-GH facilities (mean $=5.13 ; \mathrm{SD}=0.07$ ).

Moreover, we compared the four categories of the level of harm between the two groups, i.e., the treated and untreated facilities. First, for the minimal harm, GH facilities had a mean of $0.27(\mathrm{SD}=0.05)$ compared to non-GH facilities with a mean of 0.40 $(\mathrm{SD}=0.01)$. Secondly, $\mathrm{GH}$ facilities (mean $=4.21 ; \mathrm{SD}=0.26)$ had a decrease in the potential harm compared to non-GH facilities (mean $=4.45 ; \mathrm{SD}=0.06$ ). In regard to the actual harm, GH facilities were also at an advantage with a decrease in deficiency citations with a mean of $0.17(\mathrm{SD}=0.03)$ compared to non-GH facilities with a mean of $0.23(\mathrm{SD}=0.01)$. Lastly, in terms of the immediate harm, $\mathrm{GH}$ facilities (mean $=0.02 ; \mathrm{SD}=0.01$ ) had lower deficiency citations compared to non-GH facilities (mean $=0.06 ; \mathrm{SD}=0.01$ ). In all four categories, the GH model had an advantage over the NHs that did not adopt the GH model.

There is scarce research related to the impact of the GH model and the culture change movement at large on deficiency citations as a quality indicator in the literature. Grabowski et al. (2014a) described the characteristics between the NHs that did not adopt 
the culture change models, the NHs that adopted the culture change models, and the NHs that adopted the GH model and found that the culture change implementers had fewer deficiency citations compared to non-implementers. In addition, the same study found that GH NHs had fewer deficiency citations prior to the implementation of the model. To our knowledge, this is the only study that describes the GH NHs in regard to deficiency citations. Other researchers studied the impact of culture change in general on deficiency citations as a measure of quality of care. For example, Grabowski et al. (2014b) found that NHs that implemented culture change had a decrease in deficiency citations (14.6 percent) compared to NHs that did not implement a culture change model.

Beyond the traditional culture change movement, other programs such as the $\mathrm{P} 4 \mathrm{P}$ focuses on the improvement of the quality of care by providing incentives to facilities that implemented culture change concepts (as cited in Werner, Konetzka, \& Polsky, 2013) and the PEAK 2.0 program which provides incentives to NHs that adopt the culture change model for Kansas (as cited in Hermer et al., 2018). The PEAK program found similar results where NHs that joined the PEAK experienced an improvement in quality of care in terms of deficiency citations (Hermer et al., 2018). Contrarily, NHs that joined the P4P program had higher deficiency citations compared to NHs that did not join the P4P program (Werner et al., 2013).

The GH model had a higher advantage to improve the quality of care. Grabowski et al. (2014a) mentioned that the NHs that implemented the culture change had a higher quality of care before the adoption of the model. More research is needed in this area where NHs that are not better off and that implemented the GH model or other types of culture change are studied across time to describe the impact of the culture change on quality of 
care. Grabowski et al. (2014a) did not find any significant results about the impact of the GH model on deficiency citations. The researcher who attempted to analyze the impact of the GH model on deficiency citations did not have significant results just like our study; however, when they combined all culture change models, the results were significant (Grabowski et al., 2014a). This means that we need a larger sample size in the future to demonstrate the impact of the GH model on the quality of care.

\section{LIMITATIONS}

Our research has several limitations. First, the sample size was small and thus, we may not have had the statistical power to detect a true effect and we cannot generalize our findings to other NHs. This limitation was consistent with the literature on GH model and culture change in general. However, our sample counted $26 \mathrm{GH}$ organizations which was more than the sample size (15 GH organizations) that other researchers have analyzed in the past (Grabowski et al., 2016; Afendulis et al., 2016).

Secondly, GH facilities with no data prior to the GH model implementation were removed since we conducted a difference-in-difference method which requires both preand post-data. In addition, our data had missing values for the dependent variables and the independent variables. However, to test the impact of the missing data and various approached to imputing those values six scenarios were tested and the results were consistent across the scenarios (View Appendix).

Lastly, secondary data from the GH organizations and not the GH homes was used for analysis where a $\mathrm{GH}$ organization is defined as a facility that includes a legacy $\mathrm{NH}$ similar to a traditional NH and a GH home (Afendulis et al., 2016). Therefore, some results might have misled our analysis. For example, the total number of beds in non-GH facilities 
are known to be in higher number compared to GH homes. However, our descriptive summary showed the opposite where the number of beds were higher in GH facilities (mean=131.66, $\mathrm{SD}=5.56)$ than the number of beds in non-GH facilities (mean=119.95, $\mathrm{SD}=1.13$ ). This is one of the caveats of using $\mathrm{GH}$ organizations data instead of $\mathrm{GH}$ homes based data. Nevertheless, we believe that some spillover effect from the GH homes to the GH organizations might have occurred.

\section{POLICY AND PRACTICE IMPLICATIONS}

Our findings though not significant demonstrated that the GH model has the potential of having an impact on quality of care in NHs. Policies should focus on implementing and sustaining the $\mathrm{GH}$ homes and $\mathrm{GH}$ organizations. In regard to implementation, policies should focus on facilities that are contemplating the adoption of the five main GH elements including the physical structure, staffing, resident-centeredness, engagement, and family involvement. In terms of sustainability, policies should target the GH elements that were implemented but may cause the NH facility to convert back to the traditional way. GH elements which have a low probability of being well managed might be worthy of consideration for future policies. Other policies have focused on the culture change movement in general. According to Zimmerman et al. (2016), the Medicaid pay-forperformance program is committed to support NHs that adopted the culture change models including the GH model if these facilities demonstrate that they can deliver a high quality of care to their residents.

Although the presence of the OBRA-87 policy paved the way to a "better" system

that deals with long-term care with the certification process and the culture change movement, more research is needed in this area. NHs that are located in poorest 
neighborhoods were found to have more deficiency citations and thus, having a poor quality of care. Stakeholders such as policy makers, consumers, providers, and researchers in long-term care should advocate for a system benefiting the implementation and the sustainability of the various culture change models in the poor-neighborhoods with NHs high in deficiency citations. Additionally, poor neighborhoods are more likely to be associated with higher minority populations in terms of race and ethnicity. Thus, the culture change movement should transcend any financial, low-economic status, or racial obstacles. 


\section{CHAPTER 4: AIM 3}

The Green House Nursing Home Model: The Quality of Care and the Model Elements In the healthcare system, especially in the long-term care field, quality of care plays an important role. Quality of care is one of the three concepts of healthcare along with cost of health care services and access to healthcare services (Aday et al., 2004). The Institute of Medicine (IOM) defines quality of care as: "the degree to which healthcare services for individuals and populations increase the likelihood of desired health outcomes and are consistent with current professional knowledge." (Agency for Healthcare Research and Quality, 2018). With more than 1.4 million individuals residing in approximately 15,640 nursing homes (NHs) reported as of December 31st, 2014 (Centers for Medicare and Medicaid Services [CMS], 2015), quality of care is crucial to these individuals.

However, the quality in NHs has been described as poor in the past decades (Cohen et al., 2016). For instance, NH residents were more likely to experience isolation, pain that is not treated, depression, and a lack of control and decency (Cohen et al., 2016). A NH should be considered as a restful place where a person feels at home and receives the appropriate care. Usually, the population who needs NH health care services suffer from various health conditions with four major conditions known as bladder incontinence, depression, Alzheimer's type dementia, and bowel incontinence (Shi \& Singh, 2015, p. 400). 
According to the CMS (2015), 34.3\% of individuals living in NHs have a prevalence of bladder and/or bowel incontinence, $5.3 \%$ of $\mathrm{NH}$ residents experienced injuries due to falling, and $11 \%$ of $\mathrm{NH}$ residents have fallen with no injuries. Additionally, $5.1 \%$ of $\mathrm{NH}$ residents suffered from pressure ulcers stage 2 or higher, and $14.9 \%$ of $\mathrm{NH}$ residents experienced severe cognitive impairments and five Activities of Daily Living (ADL) impairments (CMS, 2015).

In response to the poor quality observed in NHs, the Nursing Home Reform Act was passed as a part of the Omnibus Reconciliation Act of 1987 (OBRA-87) (Cohen et al., 2016; Bowers et al., 2016a). This policy focused on deinstitutionalizing NHs and had an objective of resident-centeredness, leading to a movement commonly referred to as culture change (Miller et al., 2013). Overtime, this culture change movement has led to the implementation of various types including the GH model, the Eden Alternative, the Pioneer Network, the Meadowland Hills Program, Wellspring, and Planetree (Cohen et al., 2016; Ragsdale \& McDougall, 2008). For the purpose of this research, we will focus on the GH model in an effort to answer this following research question: "Which GH model elements have an effect on falls and bladder/bowel incontinence and what are these effects?" Our research objective is to analyze the impact of the GH model on the prevalence of falls and bladder/bowel incontinence.

\section{BACKGROUND}

The GH model, created in 2003, has an objective of deinstitutionalizing the long-term care sector and has a target on person-centered care (Rabig et al., 2006). A GH model includes a small home which provides a private room, bathroom, and a common area for dining purposes to its residents who can total up to 12 (Afendulis et al., 2016; Cohen et al., 2016; 
Grabowski et al., 2016; Rabig et al., 2006; Zimmerman et al., 2016). GH homes have augmented from four homes in 2003 to 174 homes in 2015 (Lum et al., 2008; Zimmerman et al., 2016). Currently, there are $237 \mathrm{GH}$ homes that are in operation and $60 \mathrm{GH}$ homes are in development in 31 states ("Find a home", 2020). Some GH homes share a license with a traditional NH (called legacy homes), while others have their own license (Afendulis et al., 2016).

A GH home uses various types of staffing that differs from that used in traditional NHs. GH staff often include a Shahbaz, a Guide, a Sage, and other non-clinical staff (Ragsdale \& McDougall, 2008). The Shahbaz is the equivalent of a certified nursing assistant (CNA) in traditional NHs (Afendulis et al., 2016). However, the Shahbazim (Plural of Shahbaz) have more tasks than do traditional CNAs (Brown et al., 2016), and are considered "universal caregivers" (Cohen et al., 2016). Shahbazim are responsible for preparing meals, doing laundry, housekeeping, and supporting residents' personal care and overall quality of life (Brown et al., 2016; Cohen et al., 2016; Rabig, et al., 2006). Shahbazim have a manager called a Guide who oversees the Shahbazim work (Rabig et al., 2006). A Sage is a community member carefully chosen by the GH Guide and staff, who volunteers to provide advice to the Shahbazim and support them in regard to decisionmaking and problem-solving duties (Ragsdale \& McDougall, 2008). A group of social workers, dieticians, nurses, and activity personnel stays near to the GH homes so they can provide services to the residents (Rabig et al., 2006; Ragsdale \& McDougall, 2008). Thus, person-centeredness, small homes, room privacy, and a special team of employees are some of the key elements of the GH model. 
In the literature, there is a mix of findings related to the overall impact of the GH model on quality of care. One study reported that the implementation of the GH model was associated with a decrease in 30-day readmission rates, bedfast residents, catheterized residents, and pressure ulcers among low-risk residents (Afendulis et al., 2016). Brown et al. (2016) found a lower turnover of staff in GH homes compared to traditional NHs. Shahbazim were also independent in terms of scheduling their own agenda compared to CNAs in traditional NHs (Brown et al., 2016). The same study showed that the culture of safety, staff stress, and staff satisfaction were not negatively affected by increased staff autonomy (Brown et al., 2016). Lastly, others found that the model lowered Medicare Part A spending (Grabowski et al., 2016).

Contrarily, other studies found that the GH model had a negative effect on the quality of care. Problems related to management, collaboration in decision-making, and training skills for the management team were observed (Bowers et al., 2016a). Daily minor issues and problems related to expertise growth for the staff were also highlighted in the literature (Bowers et al., 2016a). One of the objectives of the model was to promote more collaboration between the staff and the management team especially during decisionmaking, but research showed that the model did not deliver (Bowers et al., 2016a). Brown et al. (2016) stated that, while there was a lower turnover of staff, the Shahbazim had a heavy workload compared to the CNAs in traditional NHs.

However, a gap still exists in the literature in terms of empirically analyzing how specific elements of the GH model impact quality of care. Zimmerman and Cohen (2010) reported in their study that a consensus has been reached that the six key elements of the GH model include physical structure, staffing, dining, resident-case mix, resident- 
centeredness, and engagement (Zimmerman \& Cohen, 2010). Cohen et al. (2016) and Zimmerman et al. (2016) stressed the importance of identifying which element has an impact on quality of care. Therefore, it is important to analyze which GH components influence quality of care. For the purpose of our research, we focused on the physical structure and staffing since they are available in our dataset.

\section{CONCEPTUAL FRAMEWORK: DONABEDIAN MODEL}

In the NH literature, the Donabedian model is applied often to accomplish various projects (Horn et al., 2016; Yoon et al., 2016). For this study, the Donabedian model was applied to describe the impact of the GH model on the quality of care. The Donabedian model is comprised of three constructs including structure, process and outcome of the healthcare system (Aday et al., 2004). In the NH literature, the objective of the Donabedian's model is to show that with an effective structure, a facility will provide great care process which leads to improved outcome (Yoon et al., 2016).

Structure is defined as "the availability, organization, and financing of healthcare programs; the characteristics of the populations to be served by them; and the physical, social, and economic environments to which they are exposed." (Aday et al., 2004). Based on this definition, the availability of the GH model is incorporated in this construct. In other words, the five GH elements including the physical structure, the staff, the residentcenteredness, the engagement, and the family involvement are incorporated in the structure component. In our case, only two elements were available in the dataset which are the ones that were used.

According to Aday (2004), process is defined as "the transactions between patients and providers in the course of actual care delivery, as well as the environmental and 
behavioral transactions exacerbating health risks." In our case, the prevalence of restraints was used as a variable from the process construct as cited in the literature (Castle \& Ferguson, 2010). Based on the process definition, we understand that there is a relationship between the patients and providers and in our case, in order to protect the residents, the staff have to restrain the residents for their own good. Therefore, the restraint variable is a good indicator for the process construct. Lastly, outcome is defined as "the consequences of policies for the health and well-being of patients and the public" (Aday et al., 2004). In our case, the outcome variables included the prevalence of falls and the prevalence of bladder/bowel incontinence. In the literature, the prevalence of falls (Kane et al., 2007; Castle \& Ferguson, 2010; Horn et al., 2016) and the prevalence of bladder/bowel incontinence (Kane et al., 2007; Afendulis et al., 2016; Horn et al., 2016) were analyzed as the measures of quality of care. Thus, with an effective structure, a facility will be able to provide better health care process which will lead to improved outcomes in this case the quality of care (Yoon et al., 2016).

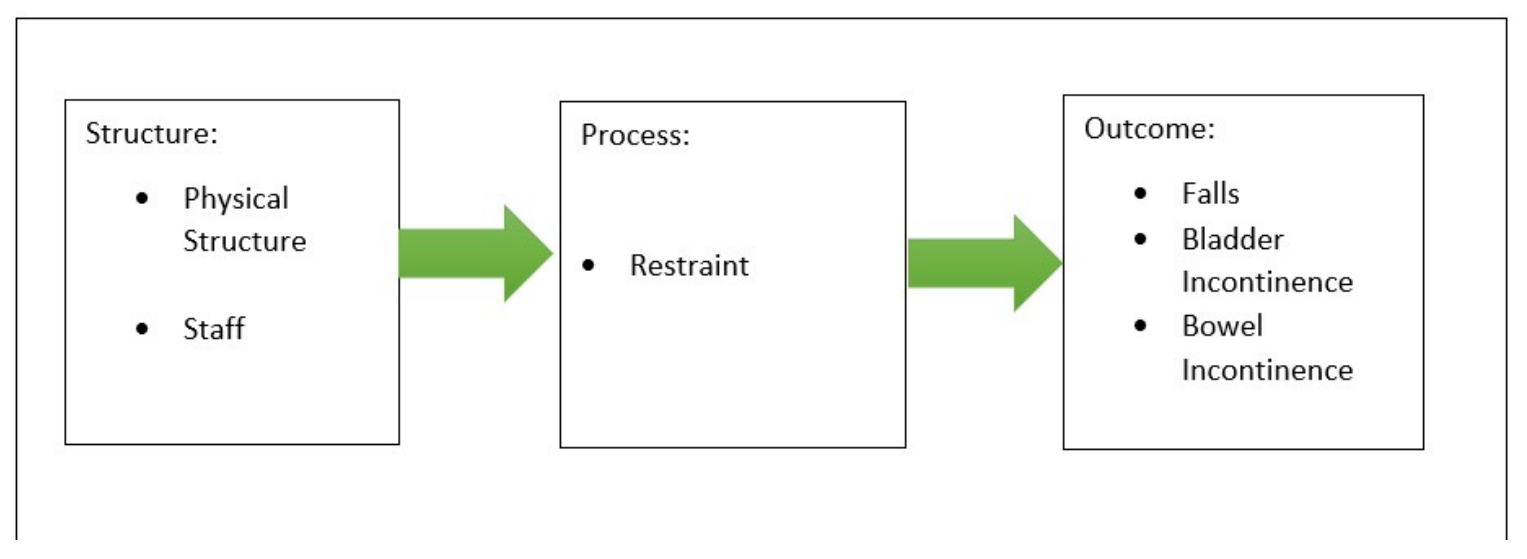

Conceptual Framework: Falls, Bladder \& Bowel Incontinence

Figure 6. Conceptual Framework 


\section{METHODS}

Data for the present project came from two sources: the Long-Term Care Focus dataset (LTCFocus) housed at the Brown University Center for Gerontology (LTCFocus, 2017) and information on NHs that implemented the GH model provided by the GH Project ("Find a home", 2020). The LTCFocus data is a combination of various datasets, including the Online Survey Certification and Reporting/Certification and Survey Provider Enhanced Reporting (OSCAR/CASPER), Minimum Data Set (MDS), Area Resource File (ARF), Residential History File (RHF), Nursing Home Compare, and State Policy Data (LTCFocus, 2017).

\section{Exclusion Criteria}

The analysis was restricted to the years 2000-2017 because of the years available in the LTCFocus dataset. We further restricted the analysis to NHs serving the general adult population aged 65 and older. We therefore excluded pediatric, veteran, and hospital-based facilities (Sharkey et al., 2011). Pediatric facilities were identified by noting NHs where the average age in the $\mathrm{NH}$ was equal or less than 21 years old and by noting NHs with names that contained children/kids/pediatrics. Veteran-based facilities were identified by observing NH names that included veterans or vets. Facilities were directly identified in the LTCFocus as hospital-based facilities, which were excluded due to concerns about noncomparability with other NHs (For example, hospital-based facilities can be much larger up to 1,098 beds - than non-hospital NHs).

Also, for non-comparability reasons, assisted living facilities were not included in the analysis. Because the statistical methods relied on matching GH NHs to non-GH NHs by state, states where there were no GH facilities were necessarily excluded from the 
dataset. Alabama, Arkansas, California, Colorado, Florida, Kansas, Massachusetts, Michigan, Mississippi, Montana, New Jersey, Ohio, Oregon, Pennsylvania, Rhode Island, Tennessee, and Virginia were included in the final dataset. In order to directly examine the effect of adopting the GH model, we excluded eleven $\mathrm{GH}$ facilities for which data was not available for the entirety of 2000-2017 (which eliminated GH facilities that were created from new using the $\mathrm{GH}$ model rather than converting to the $\mathrm{GH}$ model).

\section{Treated/Untreated Facilities}

As mentioned above, we matched GH (treated) facilities to non-GH (untreated) facilities before conducting our analyses. Matching was done using propensity scores calculated using the p-score command in Stata. With that method, a p-score for each facility (specifically, the propensity of being a GH home in or before 2017) was calculated using data from the baseline year in the data (Grabowski et al., 2014b) and the multi-facility and for-profit variables as predictors. Per Grabowski et al. (2014b), we sought a ratio of 10 matching non-GH NHs for every $\mathrm{GH}$ NH in the analysis, with matches being constrained to be in the same state as the GH home (to control for unobserved effects of state policies). In instances where more than 10 non-GH facilities were tied with the same p-score, a random sampling method was used to choose the 10 control facilities. In the one instance in which there were fewer than 10 available matches, we used all four available facilities as controls.

The resulting dataset for the analyses contained a total of 280 facilities (26 GH and 254 non-GH) across 18 years. The 26 treated facilities implemented the GH model at different points in time with 1 in 2004, 1 in 2006, 5 in 2007, 1 in 2008, 2 in 2009, 2 in 2010, 2 in 2011, 3 in 2012, 1 in 2013, 5 in 2016, and 3 in 2017. 


\section{Dependent and Independent Variables}

\section{Dependent Variables}

Fall

The first outcome variable is the prevalence of falls which is recorded in the LTCFocus dataset. The variable was aggregated at the facility, county, and state levels and was presented in percentages. The variable records if the resident had fallen within the last 30 days.

\section{Bowel Incontinence}

The second outcome variable is the prevalence of bowel incontinence which is recorded in the LTCFocus dataset. The variable was also aggregated at the facility, state, and county levels and was presented in percentages.

\section{Bladder Incontinence}

The last outcome variable is the prevalence of bladder incontinence and is also provided by the LTCFocus dataset. The dataset aggregated this variable at the facility, state, and county levels and was presented in percentages.

\section{Independent Variables}

The primary independent variables were chosen based on a combination of the GH elements available in the dataset and on the components of the Donabedian's (StructureProcess-Outcome) model. The GH model's elements include physical structure, staffing, the resident-centeredness, engagement, and family involvement (Zimmerman \& Cohen, 2010). However, only variables related to physical structure and staffing are the only elements available in the LTCFocus dataset. Physical structure was measured as the 
number of beds (following Zimmerman \& Cohen, 2010; Afendulis et al., 2016; Grabowski et al., 2016).

Staffing arrangements were measured through variables on RN hours per day, LPN hours per day, and CNA hours per day. For the GH model, the staff are Shahbazim which are an equivalent of the CNA in GH facilities (Afendulis et al., 2016). These variables are embedded within the structure component of the Donabedian's structure. The percentage of restraint was used as the process variable (Castle \& Ferguson, 2010).

\section{Covariates}

In addition to the main variables, age, gender, race, and payers will be controlled for in the analysis. Age will be defined as those who are 65 years and older. The gender variable will be considered as a categorical variable with female or male as the coded variable. The dataset provides race/ethnicity variables including White, Black, and Hispanic. The payer variable recognizes whether the resident was supported by Medicaid or Medicare. These variables were aggregated to the facility, county, and state levels.

\section{Fixed Effect Variables}

Two fixed effect variables were created for analysis purposes. A facility fixed-effect variable and a time fixed effect variable were created for the difference-in-difference method. An interaction term was also created where the treatment was multiplied with the time fixed-effect variable.

\section{Missing Values}

The LTCFocus dataset has both missing observations and instances where data was suppressed because of a low number of events (LNEs) (Needleman et al., 2018). Missing values were present for the prevalence of falls, the prevalence of bladder incontinence, the 
prevalence of bowel incontinence, age, sex, and race. Missing values (both regular and LNEs) were replaced by interpolation using the non-missing values for each facility and year as the independent variable. In other words, missing values were replaced with estimated derived from the linear time trend for each facility.

To examine robustness, we examine six alternative methods of missing values replacement, three where only LNEs were replaced and three where both LNEs and regular missing values were replaced. A brief description of each of these may be found in the Appendix. The results were consistent across all scenarios.

\section{Statistical Analysis}

The analyses were conducted using a Poisson regression model and the difference-indifference method in Stata14 as the software. The equation used was:

$$
\mathrm{Y}_{\mathrm{it}}=\beta_{0}+\beta_{1} * \mathrm{~T}_{\mathrm{i}}+\beta_{2} * \text { Year }_{\mathrm{t}}+\beta_{3} *\left(\mathrm{~T}_{\mathrm{i}} * \text { Year }_{\mathrm{t}}\right)+\beta_{4} * \mathrm{X}_{\mathrm{it}}+\mu \mathrm{GH}_{\mathrm{i}}+\Omega \mathrm{TI}_{\mathrm{t}}+\mathrm{e}_{\mathrm{it}}
$$

Where $\mathrm{i}$ represents the nursing home facility at time $\mathrm{t}$, $\mathrm{Y}$ represents the three dependent variables: the prevalence of falls, the prevalence of bladder incontinence, and the prevalence of bowel incontinence. Furthermore, $\mathrm{T}$ indicates the GH adoption variable, Year represents the series of time dummy variables, and $\mathrm{X}$ indicates the covariates (the number of beds, the RN hours per day, the LPN hours per day, the CNA hours per day, restraint, age, sex, race, payer).

Two fixed effect variables were also used including the nursing home fixed effect variable, GH and the time fixed effect variable, TI. For the facility fixed effect variable, a dichotomy variable was generated where 1 represented the adoption of the GH model and 0 indicated the non-adoption of the GH model. Regarding the time fixed effect variable, a dummy variable was created for each year where 1 represented observations present in the 
year of interest and 0 indicated observations present in other years. Interaction terms were used by multiplying the treatment variable with the time variable for the difference-indifference method totaling to a number of 18 interaction terms.

\section{RESULTS}

The average mean of $\mathrm{NH}$ residents who have fallen within 30 days was 18.77 percent, are bladder incontinent was 71.06 percent, and are bowel incontinent was 54.55 percent (See Table 4). The average mean of NH residents who were restrained was 5.27 percent. The average mean of RN hours per day was 0.41 , of LPN hours per day was 0.82 , and CNA hours per day was 2.60 . The average mean of female residents was 75.13 percent with an average mean age of 84 years old. The average of residents who were White was 89.26 percent, those who were Black was 11.29 percent and those who were Hispanic was 0.58 percent. The average of residents who had a Medicaid insurance was 57.50 percent and those who had a Medicare insurance was 11.81 percent (See Table 4).

\section{Table 4.}

\section{Descriptive Statistics}

\begin{tabular}{|l|l|l|l|l|l|}
\hline Variables & Observations & Mean & Std. Dev. & Min & Max \\
\hline $\begin{array}{l}\text { Green House } \\
\text { facilities }\end{array}$ & 5,040 & 0.034722 & 0.183094 & 0 & 1 \\
\hline \% Fallen & 5,040 & 18.77479 & 5.96817 & 0 & 58.33 \\
\hline $\begin{array}{l}\text { \% Bladder } \\
\text { Incontinence }\end{array}$ & 5,040 & 71.05547 & 12.75407 & 22.22 & 100 \\
\hline $\begin{array}{l}\text { \% Bowel } \\
\text { Incontinence }\end{array}$ & 5,040 & 54.54789 & 13.42076 & 0 & 100 \\
\hline \% Restrained & 5,040 & 5.266836 & 8.194758 & 0 & 82.35 \\
\hline $\begin{array}{l}\text { RN hours per } \\
\text { day }\end{array}$ & 5,040 & 0.412866 & 0.44006 & 0 & 19.35 \\
\hline $\begin{array}{l}\text { LPN hours } \\
\text { per day }\end{array}$ & 5,040 & 0.820713 & 0.706176 & 0 & 21.383 \\
\hline
\end{tabular}




\begin{tabular}{|l|l|l|l|l|l|}
\hline $\begin{array}{l}\text { CNA hours } \\
\text { per day }\end{array}$ & 5,040 & 2.604465 & 1.160115 & 0 & 22.65 \\
\hline $\begin{array}{l}\text { Number of } \\
\text { beds }\end{array}$ & 5,040 & 120.3538 & 78.79731 & 8 & 679 \\
\hline Average age & 5,040 & 83.67719 & 4.781195 & 55.94 & 93.02 \\
\hline \% Female & 5,040 & 75.12794 & 9.734645 & 22.89 & 100 \\
\hline \% White & 5,040 & 89.26088 & 16.92157 & 0 & 100 \\
\hline \% Black & 5,040 & 11.28807 & 17.35054 & 0 & 100 \\
\hline \% Hispanic & 5,040 & 0.58167 & 2.873911 & 0 & 57.58 \\
\hline \% Medicaid & 5,040 & 57.50314 & 23.32407 & 0 & 100 \\
\hline \% Medicare & 5,040 & 11.81451 & 11.84283 & 0 & 100 \\
\hline $\begin{array}{l}\text { Year of the } \\
\text { dataset }\end{array}$ & 5,040 & 2008.5 & 5.188642 & 2000 & 2017 \\
\hline
\end{tabular}

Table 4

\section{Dependent Outcome - Prevalence of Falls}

We did not find any statistically significant result to suggest that there is a difference between GH facilities and non-GH facilities in regard to the prevalence of falls (IRR: 1.072; P-value: 0.101). In other words, there is no statistically significant result to suggest that there is a GH model impact on the prevalence of falls. Although, our findings were not statistically significant, there was an increasing trend of the prevalence of falls in GH facilities compared to non-GH facilities across the years (See Figure 7). 


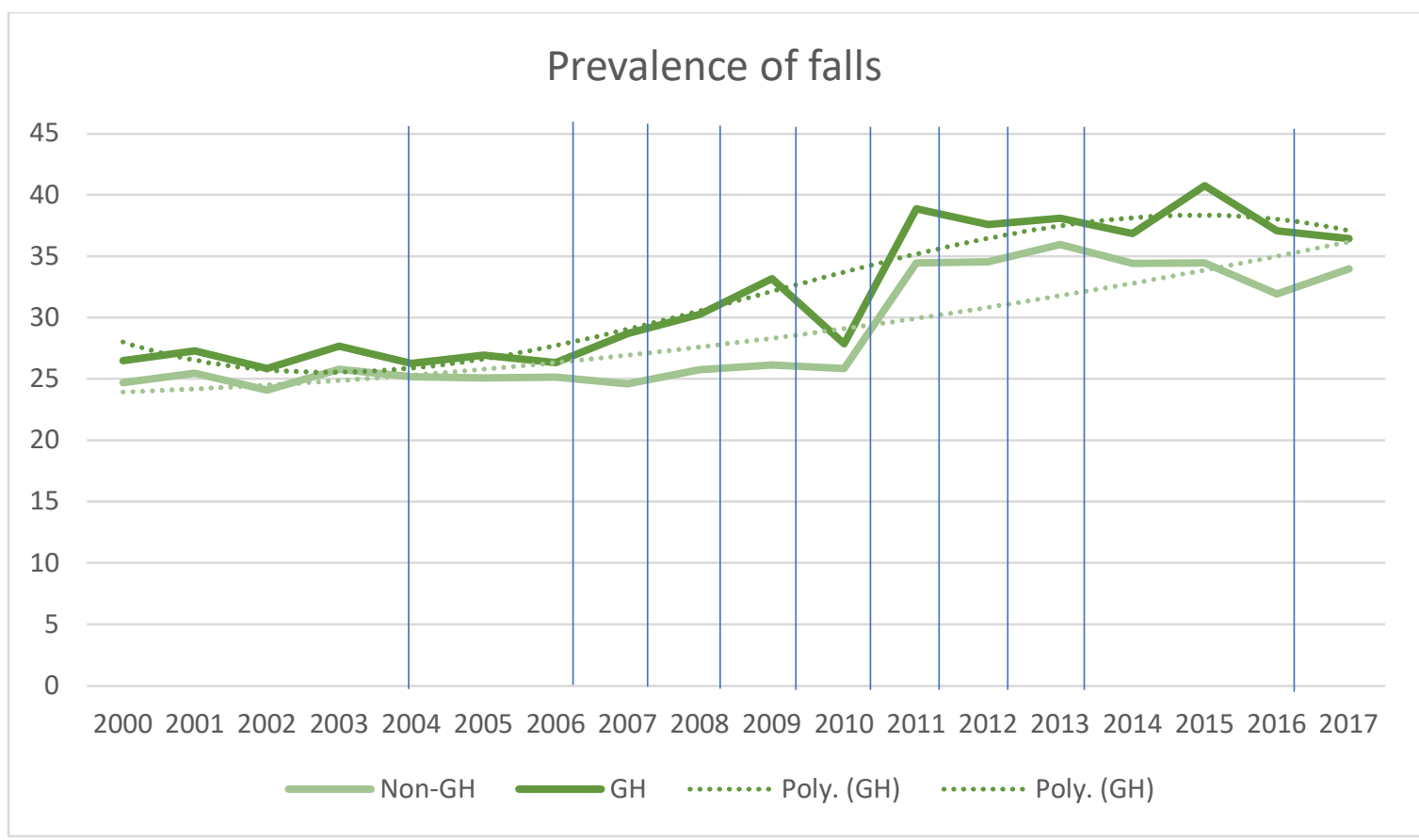

Prevalence of falls.

Figure 7. Prevalence of falls. N.B. Years of GH Implementation: 2004, 2006, 2007, 2008, 2009, 2010, 2011, 2012, 2013, 2016, \&2017.

\section{Independent Variables}

Structure Variables

Regarding the structure construct, the number of beds were less likely to be associated with the prevalence of falls in $\mathrm{GH}$ facilities compared to non-GH facilities (IRR: 0.9991; p-value: <.001) while the association between the staff hours per day and the prevalence of falls was not statistically significant with RN hours per day (IRR: 0.992; pvalue: 0.396), LPN hours per day (IRR: 0.9986; p-value: 0.805), and CNA hours per day (IRR: 0.9953; p-value: 0.18). Thus, we could not suggest any association between the staff hours per day and the prevalence of falls. 


\section{Process Variables}

The number of restrained residents were less likely to be associated with the prevalence of falls in GH facilities comparing to non-GH facilities (IRR: 0.9984; p-value: $0.001)$.

\section{Covariates}

Female residents were less likely to be associated with the prevalence of falls in GH facilities compared to non-GH facilities (IRR: 0.9972; p-value: $<.001$ ). The association between age and the prevalence of falls was not statistically significant even though the results suggest that the prevalence of falls was less likely to be associated with age in GH facilities compared to non-GH facilities (IRR: 0.9993; p-value:0.54). White residents were more likely to be associated with the prevalence of falls in $\mathrm{GH}$ facilities in comparison to residents in non-GH facilities (IRR: 1.0014; p-value: <.001). Residents with Medicaid (IRR: 0.998; p-value: <.001) and Medicare (IRR: 0.996; p-value:<.001) were less likely to be associated with the prevalence of falls in GH facilities than non-GH facilities.

\section{Table 5.}

Poisson Regression Model Predicting the Number of Falls

\begin{tabular}{|l|l|l|l|l|}
\hline Variables & $\begin{array}{l}\text { Incidence Rate } \\
\text { Ratio }\end{array}$ & \multicolumn{2}{|l|}{$95 \%$ Confidence Interval } & $\mathrm{p}$-value \\
\hline $\begin{array}{l}\text { Green } \\
\text { House } \\
\text { facilities }\end{array}$ & 1.072433 & 0.986523 & 1.165824 & 0.101 \\
\hline $\begin{array}{l}\text { Number of } \\
\text { beds }\end{array}$ & 0.999062 & 0.998963 & 0.999161 & $<.001$ \\
\hline $\begin{array}{l}\text { RN hours } \\
\text { per day }\end{array}$ & 0.992791 & 0.976328 & 1.009532 & 0.396 \\
\hline $\begin{array}{l}\text { LPN hours } \\
\text { per day }\end{array}$ & 0.998556 & 0.98718 & 1.010063 & 0.805 \\
\hline
\end{tabular}




\begin{tabular}{|l|l|l|l|l|}
\hline $\begin{array}{l}\text { CNA } \\
\text { hours per } \\
\text { day }\end{array}$ & 0.995333 & 0.988552 & 1.002159 & 0.18 \\
\hline $\begin{array}{l}\text { \% } \\
\text { Restrained }\end{array}$ & 0.998413 & 0.997482 & 0.999344 & 0.001 \\
\hline $\begin{array}{l}\text { Average } \\
\text { age }\end{array}$ & 0.99931 & 0.997109 & 1.001516 & 0.54 \\
\hline $\begin{array}{l}\text { \% Female } \\
\text { \% White }\end{array}$ & 0.997176 & 0.996251 & 0.998101 & $<.001$ \\
\hline$\%$ Black & 0.999218 & 1.000761 & 1.001953 & $<.001$ \\
\hline $\begin{array}{l}\text { \% } \\
\text { Hispanic }\end{array}$ & 0.997639 & 0.998644 & 0.999793 & 0.008 \\
\hline $\begin{array}{l}\text { \% } \\
\text { Medicaid }\end{array}$ & 0.998349 & 0.997986 & 0.998712 & $<.001$ \\
\hline $\begin{array}{l}\text { \% } \\
\text { Medicare }\end{array}$ & 0.996285 & 0.995621 & 0.99695 & $<.001$ \\
\hline Constant & 24.70576 & 20.8027 & 29.34111 & $<.001$ \\
\hline
\end{tabular}

Table 5

\section{Dependent Outcome - Prevalence of Bladder Incontinence}

There was no association between the $\mathrm{GH}$ model and the prevalence of bladder incontinence. We did not find any statistically significant results to suggest that there is a difference between GH facilities and non-GH facilities (IRR: 0.959; P-value: 0.0608). However, a decreasing trend among GH facilities was observed across the years (See Figure 8). 


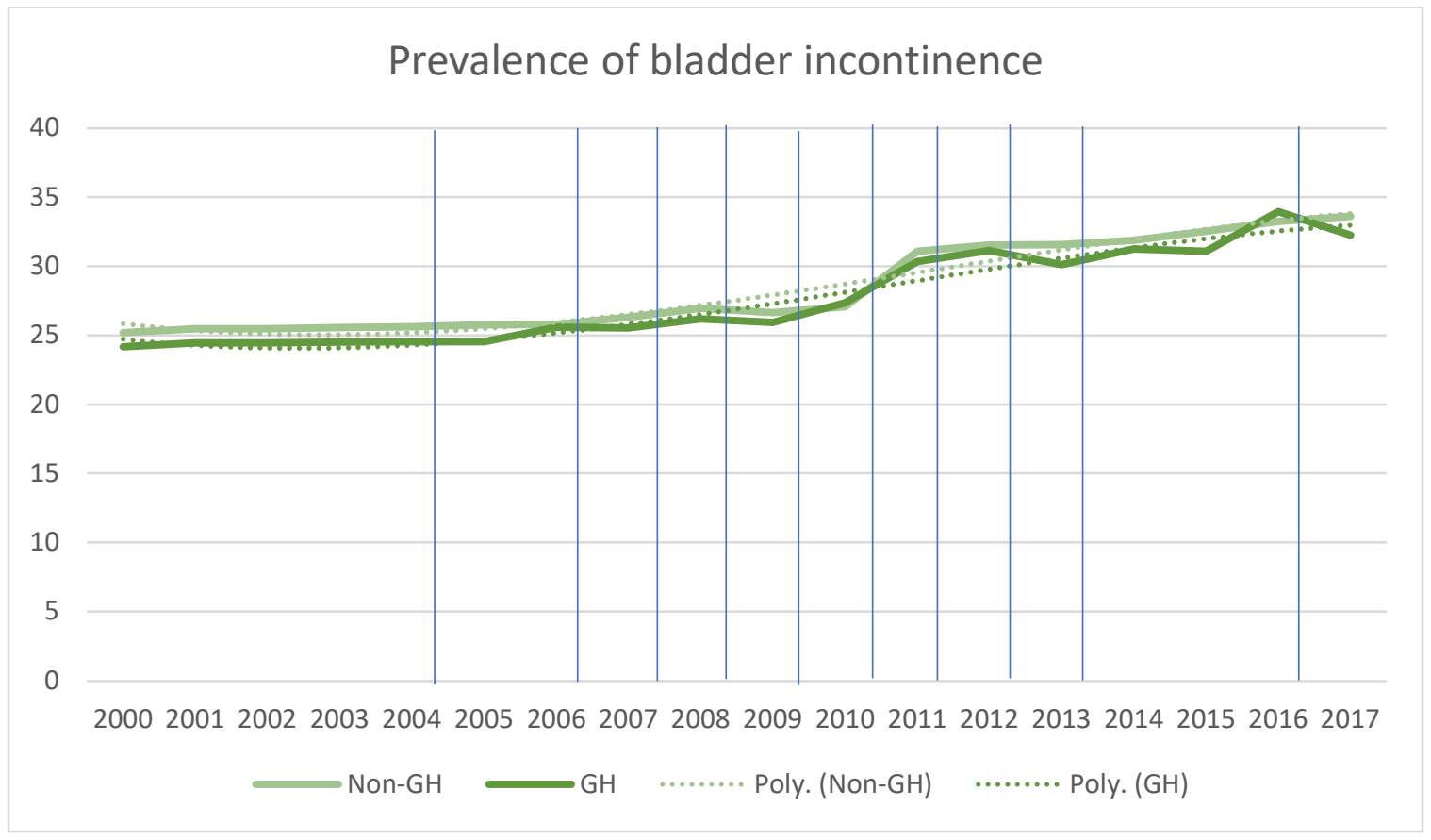

Prevalence of Bladder Incontinence

Figure 8. Prevalence of Bladder Incontinence. N.B. Years of GH implementation:

2004,2006,2007,2008,2009,2010,2011,2012,2013,2016, \&2017

\section{Independent Variables}

Structure Variables

Concerning the structure component, the number of beds were more likely to be associated with the prevalence of bladder incontinence in $\mathrm{GH}$ facilities compared to nonGH facilities (IRR: 1.0003; p-value: <.001) while the association between the staff hours per day and the prevalence of bladder incontinence was not statistically significant with RN hours per day (IRR: 0.997; p-value: 0.46) and CNA hours per day (IRR: 1.0001; pvalue: 0.958 ). Therefore, we could not suggest any association between the RN hours per day and the CNA hours per day and the prevalence of bladder incontinence. However, the LPN hours per day were more likely to be associated with the prevalence of bladder incontinence (IRR: 1.0069; p-value: 0.014). 


\section{Process Variable}

Regarding the process construct, the number of restrained residents were more likely to be associated with the prevalence of bladder incontinence in $\mathrm{GH}$ facilities comparing to non-GH facilities (IRR: 1.0014; p-value: <.001).

\section{Covariates}

For the other covariates, sex, age, and race were more likely to be associated with the prevalence of bladder incontinence while the insurance type was less likely to be associated with the prevalence of bladder incontinence. Female residents were more likely to be associated with the prevalence of bladder incontinence in $\mathrm{GH}$ facilities compared to non-GH facilities (IRR: 1.0027; p-value: $<.001$ ). Age was more likely to be associated with the prevalence of bladder incontinence in $\mathrm{GH}$ facilities compared to non-GH facilities (IRR: 1.0079; p-value:<.001). White residents were more likely to be associated with the prevalence of bladder incontinence in $\mathrm{GH}$ facilities in comparison to non-GH facilities (IRR: 1.0006; p-value: <.001). Residents with Medicaid (IRR: 0.9991; p-value: <.001) and Medicare (IRR: 0.9989; p-value:<.001) were less likely to be associated with the prevalence of bladder incontinence in GH facilities than non-GH facilities.

\section{Table 6.}

Poisson Regression Model Predicting the Number of Bladder Incontinence

\begin{tabular}{|l|l|l|l|l|}
\hline Variable & Incidence Rate Ratio & \multicolumn{2}{|l|}{$\begin{array}{l}\text { 95\% Confidence } \\
\text { Interval }\end{array}$} & p-value \\
\hline $\begin{array}{l}\text { Green House } \\
\text { facilities }\end{array}$ & 0.959328 & 0.917563 & 1.002995 & 0.068 \\
\hline Number of beds & 1.000335 & 1.000288 & 1.000381 & $<.001$ \\
\hline RN hours per day & 0.996765 & 0.988231 & 1.005373 & 0.46 \\
\hline LPN hours per day & 1.006926 & 1.001376 & 1.012506 & 0.014 \\
\hline
\end{tabular}




\begin{tabular}{|l|l|l|l|l|}
\hline CNA hours per day & 1.000091 & 0.996698 & 1.003495 & 0.958 \\
\hline Average age & 1.007923 & 1.006748 & 1.0091 & $<.001$ \\
\hline \% Restrained & 1.00183 & 1.001369 & 1.002291 & $<.001$ \\
\hline \% Female & 1.002704 & 1.002216 & 1.003192 & $<.001$ \\
\hline \% White & 1.00058 & 1.000278 & 1.000883 & $<.001$ \\
\hline \% Black & 1.001512 & 1.001222 & 1.001803 & $<.001$ \\
\hline \% Hispanic & 0.994451 & 0.993096 & 0.995807 & $<.001$ \\
\hline \% Medicaid & 0.999077 & 0.998887 & 0.999266 & $<.001$ \\
\hline \% Medicare & 0.998892 & 0.998553 & 0.999231 & $<.001$ \\
\hline Constant & 25.20064 & 22.99881 & 27.61327 & $<.001$ \\
\hline
\end{tabular}

Table 6

\section{Dependent Outcome - Prevalence of Bowel Incontinence}

We found a statistically significant result difference between GH facilities and non-GH facilities when it comes to the prevalence of bowel incontinence (IRR: 0.920; P-value: 0.002). These findings suggest that there is a GH model impact on the prevalence of bowel incontinence. Similar to the prevalence of bladder incontinence, a decreasing trend was also observed among GH facilities across the years (See Figure 9). 


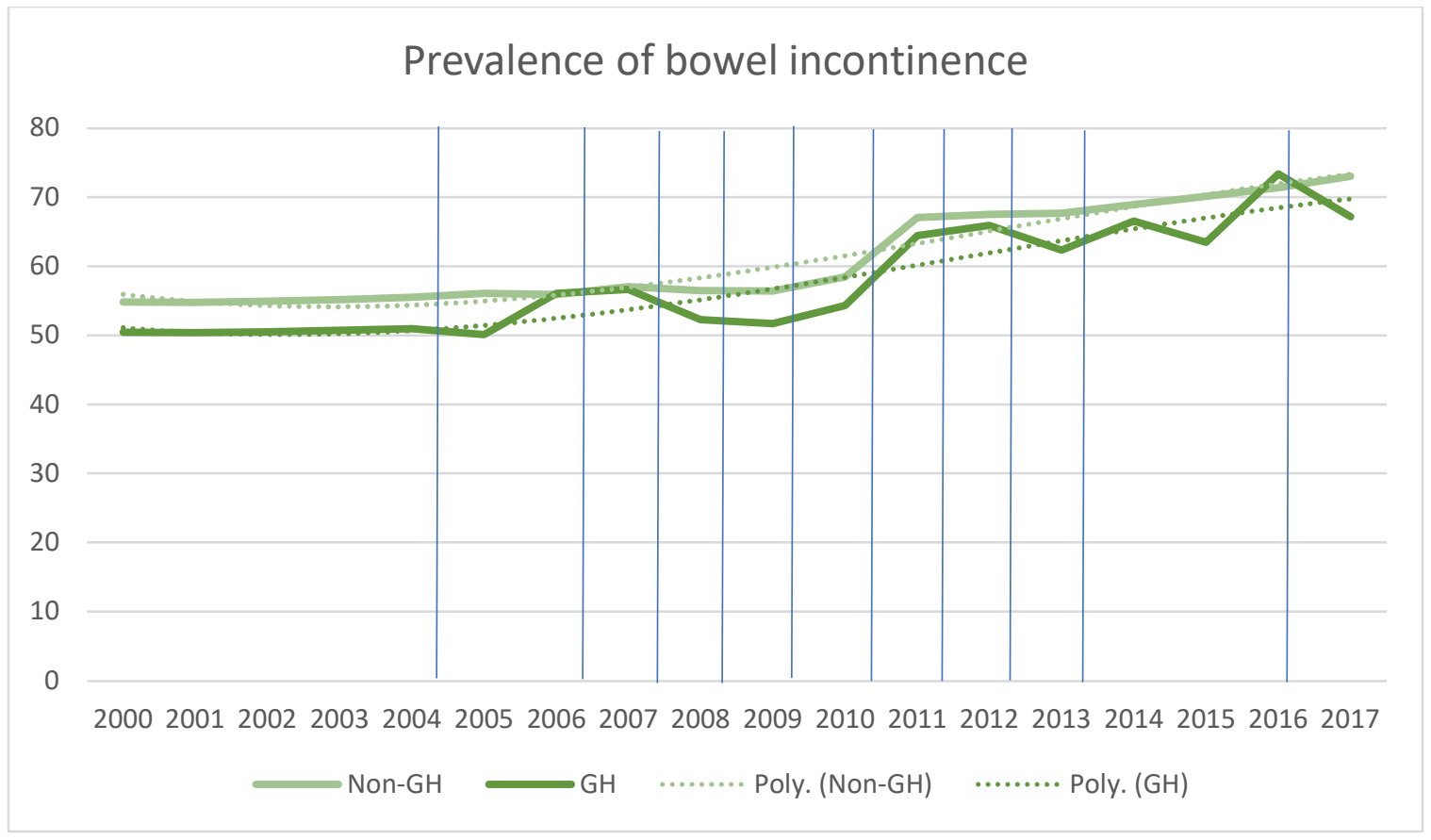

Prevalence of Bowel Incontinence

Figure 9. Prevalence of Bowel Incontinence. N.B. Years of GH implementation:

2004,2006,2007,2008,2009,2010,2011,2012,2013,2016,\&2017.

\section{Independent Variables}

Structure Variables

Concerning the structure component, the number of beds were more likely to be associated with the prevalence of bowel incontinence in $\mathrm{GH}$ facilities compared to nonGH facilities (IRR: 1.0005; p-value: <.001) while the association between the staff hours per day and the prevalence of bowel incontinence was not statistically significant with LPN hours per day (IRR: 1.0056; p-value: 0.075), and CNA hours per day (IRR: 1.0002; pvalue: 0.936 ). Thus, we could not suggest any association between the LPN hours per day and CNA hours per day and the prevalence of bowel incontinence. However, the RN hours per day were less likely to be associated with the prevalence of bowel incontinence in GH facilities than in non-GH facilities (IRR: 0.9780; p-value: <.001). 


\section{Process Variable}

In regard to the process construct, the number of restrained residents were more likely to be associated with the prevalence of bowel incontinence in $\mathrm{GH}$ facilities comparing to non-GH facilities (IRR: 1.0046; p-value: <.001).

\section{Covariates}

In terms of the covariates, female residents were more likely to be associated with the prevalence of bowel incontinence in GH facilities compared to non-GH facilities (IRR: 1.0049; p-value: <.001). Age was less likely to be associated with the prevalence of bowel incontinence in GH facilities compared to non-GH facilities (IRR: 0.9943; p-value: <.001). The association between White residents and the prevalence of bowel incontinence was not statistically significant (IRR: 0.9997; p-value: 0.060). Residents with Medicaid were less likely to be associated with the prevalence of bowel incontinence in GH facilities than non-GH facilities (IRR: 0.9985; p-value: <.001). The association between residents with Medicare and the prevalence of bowel incontinence was not statistically significant (IRR: 1.0004; p-value: 0.074).

\section{Table 7.}

Poisson Regression Model Predicting the Number of Bowel Incontinence

\begin{tabular}{|l|l|l|l|l|}
\hline Variable & Incidence Rate Ratio & $95 \%$ Confidence Interval & $\mathrm{p}$-value \\
\hline $\begin{array}{l}\text { Green House } \\
\text { facilities }\end{array}$ & 0.919763 & 0.872796 & 0.969257 & 0.002 \\
\hline Number of beds & 1.000458 & 1.000406 & 1.00051 & $<.001$ \\
\hline RN hours per day & 0.978048 & 0.967857 & 0.988346 & $<.001$ \\
\hline LPN hours per day & 1.005629 & 0.999426 & 1.011871 & 0.075 \\
\hline CNA hours per day & 1.000157 & 0.996331 & 1.003998 & 0.936 \\
\hline
\end{tabular}




\begin{tabular}{|l|l|l|l|l|}
\hline \% Restrained & 1.004618 & 1.00411 & 1.005125 & $<.001$ \\
\hline Average age & 0.994321 & 0.99304 & 0.995604 & $<.001$ \\
\hline \% Female & 1.004854 & 1.004298 & 1.005411 & $<.001$ \\
\hline \% White & 0.999684 & 0.999355 & 1.000013 & 0.060 \\
\hline \% Black & 1.003039 & 1.002724 & 1.003353 & $<.001$ \\
\hline \% Hispanic & 0.995598 & 0.994121 & 0.997077 & $<.001$ \\
\hline \% Medicaid & 0.998535 & 0.998316 & 0.998754 & $<.001$ \\
\hline Constant & 1.000351 & 0.999966 & 1.000736 & 0.074 \\
\hline
\end{tabular}

Table 7

\section{DISCUSSION}

\section{The prevalence of falls}

Our findings suggest that there is not a statistical difference between GH facilities and non-

GH facilities in regard to the prevalence of falls (IRR: 1.072; P-value: 0.101). In other words, there is no statistically significant impact of the GH model on the prevalence of falls. Even though our findings were non-significant, there is a higher trend of the prevalence of falls in GH facilities compared to non-GH facilities across the years which is consistent with the literature. Kane et al. (2007) found that the GH facilities (OR: 2.10) tended to have more falls than traditional NHs (OR:2.04) which can be explained by the presence of active residents in GH facilities compared to traditional NHs (Grabowski et al., 2014b; Afendulis et al., 2016).

On a larger scale, $\mathrm{NH}$ facilities that implemented other type of culture change such as the Eden Alternative found that individuals living in those facilities experienced more 
falls than those in control facilities (Coleman et al., 2002). The literature on culture change also supports the fact that there is an increase in falls in NHs that adopted culture change (Fritsch et al., 2009; Shura et al., 2011; Bourgeois et al., 2015; Hermer et al., 2017). To correct this problem in GH facilities, Horn et al. (2016) found that communication among the staff decreased the number of falls (Horn et al., 2016).

\section{The prevalence of bladder and bowel Incontinence}

There was no association between the impact of the GH model and the prevalence of bladder incontinence. We did not find any statistically significant result to propose that there is a difference between GH facilities and non-GH facilities (IRR: 0.959; P-value: 0.0608). However, a decreasing trend among GH facilities was observed across the years. Regarding the prevalence of bowel incontinence, there was a statistically significant result to suggest that there is a difference between GH facilities and non-GH facilities when it comes to the prevalence of bowel incontinence (IRR: 0.920; P-value: 0.002). Similar to the prevalence of bladder incontinence, a decreasing trend was also observed among GH facilities across the years.

Kane et al. (2007) found that an increase in the prevalence of incontinence and Afendulis et al. (2016) did not find any impact of the GH model on the prevalence of incontinence. Both papers did not distinguish the types of incontinence, i.e. either the prevalence of bowel or bladder incontinence. This might be the reason we observed a decreasing trend across the years among the GH facilities. Also, the years of analysis are different for both papers and our years of analysis which might contribute to the difference in results. 
Lastly, while our analysis focused on the $\mathrm{GH}$ organizations and not the $\mathrm{GH}$ facilities, Kane et al. (2007) focused on the GH homes. This lack of data on GH homes was considered as a limitation. In addition, our data was aggregated at the facility level; in other words, we did not have data at the resident level. These various reasons might explain the difference that we observed between our findings on the prevalence of bladder and bowel incontinence and the findings of other researchers in the field.

When it comes to other types of the culture change movement, Kruschke (2006) found that there was a decrease in the prevalence of incontinence (without distinguishing which type of incontinence) in NHs that implemented the Eden Alternative compared to those that did not implement the model. Stone et al. (2002) did not find any difference related to the prevalence of bladder/bowel incontinence between residents in the Wellspring facilities and residents in control facilities.

Based on our research objective to identify the impact of the GH elements on the quality of care, we were able to find significant results on the impact of the two GH elements including the physical structure and the staff on the quality of care. Regarding the prevalence of falls, the physical structure was less likely to be associated with the prevalence of falls while the same element was more likely to be associated with the prevalence of bladder/bowel incontinence in GH facilities compared to non-GH facilities.

With these mixed findings, it is difficult to conclude that the physical structure provide a positive or negative effect on the quality of care indicators. With the staff variables, we were not able to find any statistically significant difference between the RN hours per day, the LPN hours per day, and the CNA hours per day, and the prevalence of falls. There was also no statistically significant difference between the RN hours per day 
and the CNA hours per day, and the prevalence of bladder incontinence. We were also not able to find any statistically significant difference between the LPN hours per day and CNA hours per day, and the prevalence of bowel incontinence. However, an association was statistically significant between the LPN hours per day and the prevalence of bladder incontinence, and the RN hours per day and the prevalence of bowel incontinence. These findings might also suggest that this GH element might have an impact on the quality of care among NH residents.

However, we should be careful with the interpretation of these findings, since we analyzed GH organizations which might have the same physical structure and staff as traditional NHs. In other words, the GH homes might report their assessments along with the legacy NH which makes it impossible to distinguish the impact of each GH element or the GH model in general on quality of care.

\section{LIMITATIONS}

Our research encountered various limitations which most were also observed in the GH model and the culture change literature. First, our sample size was small and thus, we could not generalize our findings to other NHs. This limitation was consistent with the literature on GH model and culture change in general. However, our sample counted $26 \mathrm{GH}$ organizations which was more than the sample size (15 GH organizations) that other researchers have analyzed (Grabowski et al., 2016; Afendulis et al., 2016).

Secondly, GH facilities with no data prior to the GH model implementation were removed since we conducted a difference-in-difference method which requires to have preand post-data. In addition, our data had missing values for the dependent variables and the 
independent variables. Thus, six scenarios were created in order to correct the problem, and the results were almost the same across the scenarios.

Lastly, secondary data from the GH organizations and not the GH homes was used for analysis where a $\mathrm{GH}$ organization is defined as a facility that includes a legacy $\mathrm{NH}$ similar to a traditional NH and a GH home (Afendulis et al., 2016).

\section{POLICY AND PRACTICE IMPLICATIONS}

Policies related to culture change, especially GH model, should focus on the extension of these models to states that are culture change free. In regard to the GH model, the policies should target the five main GH elements including the physical structure, staff, residentcenteredness, engagement, and family involvement. According to Zimmerman et al. (2016), the Medicaid pay-for-performance program is committed to support NHs that adopted the culture change models including the GH model if these facilities demonstrate that they can deliver a high quality of care to their residents. However, the literature has shown that the implementation of the culture change models happens when the facilities want to increase their Medicare mix-case (Lepore et al., 2015), and when there is an increase of Medicaid payment rates (Grabowski et al., 2014a).

Even though it has been a long journey from the OBRA- 87 era to the culture change movement, the fight against poor quality is not over. Various challenges including financial issues, accessibility problems, racial disparities, and obstacles related to low-economic status should continue to be addressed by stakeholders such as consumers, lawmakers, providers, and researchers in the long-term care field. Policies should target to implement the GH model in NHs located in low-economic neighborhoods, predominantly minorities in terms of race, and rural places. Future research should analyze the association between 
the low economic and racial status, and the GH model. The outcome of this research should inform the long-term care field and the public on which measures to incorporate. 


\section{CHAPTER 5}

\section{CONCLUSION}

\section{The GH Model}

The GH model is one type of the culture change movement with an objective of deinstitutionalizing the long-term care field and focusing on person-centered care. Different GH elements exist including the physical structure, staffing, residentcenteredness, engagement, and family involvement. The GH model and other culture change types have been developed to correct the issues that NH residents experienced in NHs.

In our first aim, we found that the GH model had positive and negative findings in terms of its impact on the quality of care. For example, Afendulis et al. (2016) reported a decrease in 30-day readmission rates, bedfast residents, catheterized residents, and pressure ulcers among low-risk residents. Another study found a lower turnover of staff in GH homes compared to traditional NHs (Brown et al., 2016). One of the objectives of the model was to promote more collaboration between the staff and the management team especially during decision-making, but research showed that the model did not deliver (Bowers et al., 2016a). However, there was a lack of empirical impact of the GH model elements on quality of care. With these findings from the literature, we decided to empirically analyze the impact of the GH model elements, especially the physical structure and staffing (since these elements were the only ones present in the dataset) on quality of care. The quality of care was examined using the quality of care indicators 
including the number of deficiency citations, the prevalence of falls, and the prevalence of bladder/bowel incontinence. Our findings were able to show the impact of the GH model on quality of care may provide benefits to $\mathrm{NH}$ residents.

Even though the impact of the GH model on the number of deficiency citations did not provide any statistically significant results, the direction of the results showed that GH homes are less likely to receive deficiency citations compared to non-GH homes. In addition, our descriptive statistics showed that the various levels of harm were lower in GH homes compared to the levels of harm in non-GH homes. Grabowski et al. (2014a) had significant results in regard to the effect of the culture change model on the deficiency citations with a 14.6 percent decrease. However, Grabowski et al. (2014a) did not find any statistically difference between GH homes and non-GH facilities. Moreover, our research might be the first one to have described the levels of harm in terms of deficiency citations in the GH literature. More evidence is needed in this field so that long-term care experts, policy-makers, consumers, and other stakeholders understand the benefits and the disadvantages of the GH model, and thus the culture change models at large.

Even though the impact of the GH model on the number of falls did not yield significant results, the trends over the years showed that more falls were more likely to occur in GH facilities compared to non-GH facilities. Kane et al. (2007) found similar results with an odds ratio being 2.10. These findings mean that residents in GH homes are more likely to be active and thus fall compared to residents in non-GH homes (Grabowski et al., 2014b; Afendulis et al., 2016). With these results, fall prevention programs have been established in most GH homes (Cohen et al., 2016). 
In regard to the prevalence of bowel incontinence, our results show that GH facilities were less likely to have residents with bowel incontinence compared to non-GH facilities. The impact of the GH model on the prevalence of bladder incontinence was not statistically significant; however, the direction of the results was positive. In other words, the trend demonstrated that GH facilities are less likely to have residents with bladder incontinence compared to non-GH facilities. In the GH literature, Kane et al. (2007) found that an increase in the prevalence of incontinence and Afendulis et al. (2016) did not find any impact of the GH model on the prevalence of incontinence. Both studies did not distinguish the types of incontinence, i.e. either the prevalence of bowel or bladder incontinence. The non-distinction between the bladder incontinence and bowel incontinence makes it impossible to compared with our results.

Even though limitations were present in the analysis of the GH impact on quality of care including a small size sample and a reduced number of residents per home which led to a lack of generalizability to the rest of NHs, the little evidence demonstrates that the impact of the GH model on quality of care shows positive effects. Therefore, the implementation of the GH home model promises a better quality of care. Though it has been a long journey from the complaints of the consumer group in the 1980s to the adoption of the OBRA- 87 to the creation of the culture change movement and to the development of the GH model (Cohen et al., 2016), the long-term care field still has a long way ahead of it.

\section{Policy and Practice Recommendations}

Our findings show that even though the impact of the GH model was not significant for most of the quality of care indicators, policymakers should focus on the implementation 
and sustainability of the GH model. In regard to implementation, policies should focus on facilities that are contemplating the adoption of the five main GH elements including the physical structure, staffing, resident-centeredness, engagement, and family involvement. In terms of sustainability, policies should target the GH elements that were implemented but may cause the NH facility to convert back to the traditional way. GH elements which have a low probability of being well managed might be worthy of consideration for future policies. The GH model should be extended to other states where the GH model is not present.

Stakeholders such as policy makers, consumers, providers, and researchers in longterm care should also advocate for a system benefiting the implementation and the sustainability of the various culture change models in the poor-neighborhoods with NHs high in deficiency citations. Additionally, poor neighborhoods are more likely to be associated with higher minority populations in terms of race and ethnicity. Thus, the GH model should transcend any financial, low-economic status, or racial obstacles.

The fight against poor quality is not over, various challenges including financial issues, accessibility problems, racial disparities, and obstacles related to low-economic status should continue to be addressed by stakeholders such as the NH management, consumers, lawmakers, providers, and researchers in the long-term care field.

\section{Future Research}

Future research should target which element of the GH model has a positive impact on quality of care so that implementation of the model would be less difficult. It should also analyze the impact of each element of the GH model on quality of care since in the study 
was limited to two elements, but there are other GH elements to consider. More research should also analyze the association between the low economic and racial status, and the GH model. The outcome of this research should inform the long-term care field and the public on which measures to incorporate.

Future research will benefit from "complete" data in terms of quality of care indicators which will help to advance our understanding of the impact of the GH model on quality of care. To make this possible, these "complete" data about the GH model should be made available to the public. Zimmerman et al. (2016) also agrees with the need of reporting the data about culture change models, similar to the MDS quality indicators.

The GH model is a model that allows residents to be autonomous, the staff to be more empowered, and permits family members to get involved (Cohen et al., 2016). This model has a holistic view of the care centered to the patient; that is, a whole team of providers including the Shahbazim, the directors of nursing, RNs, LPNs, Guide, and physicians (Ragsdale \& McDougall, 2008) work together to improve the quality of care of their residents with a heavy responsibility based on the Shahbazim or CNAs (Brown et al., 2016). However, it is crucial to understand the impact of the GH elements on the quality of care so that if any facility interested in adopting the model may have a better understanding on which element to focus on. Plus, the cost of engaging with each GH element may be of interest as well for those facilities that are in the contemplation stage. 


\section{REFERENCES}

References with an asterisk represent studies present in the systematic review.

Administration on Aging. (2018). Profile of Older Americans: 2017. Available at: https://www.acl.gov/sites/default/files/Aging\%20and\%20Disability\%20in\%20Am erica/2017OlderAmericansProfile.pdf

Aday, L.A., Begley C.E., Lairson, D.R., \& Balkrishan R. (2004). Evaluating the healthcare system: effectiveness, efficiency, and equity. Chicago: Health Administration Press.

*Afendulis, C. C., Caudry, D. J., O’Malley, A. J., Kemper, P., \& Grabowski, D. C. (2016). Green house adoption and nursing home quality. Health Services Research, 51 Suppl 1, 454-474. Doi:10.1111/1475-6773.12436

Agency for Healthcare Research and Quality. (2018). Understanding quality measurement. Retrieved from https://www.ahrq.gov/professionals/quality-patientsafety/quality-resources/tools/chtoolbx/understand/index.html

Ausserhofer, D., Deschodt, M., De Geest, S., van Achterberg, T., Meyer, G., Verbeek, H., . . Engberg, S. (2016). "There's no place like home": a scoping review on the impact of homelike residential care models on resident-, family-, and staff-related outcomes. Journal of the American Medical Directors Association, 17(8), 685693. doi:10.1016/j.jamda.2016.03.009

Berridge, C., Tyler, D. A., \& Miller, S. C. (2018). Staff empowerment practices and CNA retention: findings from a nationally representative nursing home culture change survey. Journal of Applied Gerontology, 37(4), 419-434. doi:10.1177/0733464816665204

Bourgeois, M.S., Brush, J., Elliot, G., \& Kelly, A. (2015). Join the revolution: How Montessori for aging and dementia can change long-term care culture. Seminars in Speech and Language, 36(3), 209-214. doi:/10.1055/s-0035-1554802.

*Bowers, B., \& Nolet, K. (2011). Empowering direct care workers: Lessons learned from the green house model. Seniors Housing \& Care Journal, 19(1), 109-120

*Bowers, B. J., \& Nolet, K. (2014). Developing the green house nursing care team: 
variations on development and implementation. Gerontologist, 54 Suppl 1, S5364. doi:10.1093/geront/gnt109

Bowers, B., Nolet, K., \& Jacobson, N. (2016a). Sustaining culture change: Experiences in the green house model. Health Services Research, 51 Suppl 1, 398-417. doi:10.1111/1475-6773.12428

*Bowers, B., Roberts, T., Nolet, K., \& Ryther, B. (2016b). Inside the green house "black box": Opportunities for high-quality clinical decision making. Health Services Research, 51 Suppl 1, 378-397. doi:10.1111/1475-6773.12427

*Brown, P. B., Hudak, S. L., Horn, L.W., Cohen, L.W., Reed, D. A., \& Zimmerman, S. (2016). Workforce characteristics, stress, and satisfaction among staff in green house and other nursing homes. Health Services Research, 51 Suppl 1, 418-432. doi: $10.1111 / 1475-6773.12431$

Brown University School of Public Health. (2017). LTCFocUS.org. Retrieved from: http://ltcfocus.org.

Brune, K. (2011). Culture change in long term care services: Eden-greenhouse-aging in the community. Educational Gerontology, 37(6), 506-525. doi:10.1080/03601277.2011.570206

Castle, N.G., \& Myers, S. (2006). Mental health care deficiency citations in nursing homes and caregiver staffing. Administration and policy in mental health and mental health services research, 33(2), 215-225. doi: 10.1007/s10488-006-0038-2

Castle, N. G., Engberg, J., \& Men, A. (2007). Variation in the use of nursing home deficiency citations. Journal for healthcare quality, 29(6), 12-23.

Castle, N.G., \& Ferguson, J.C. (2010). What is nursing home quality and how is it measured? The Gerontologist, 50(4), 426-442. doi: 10.1093/geront/gnq052.

Castle, N. G., Wagner, L. M., Ferguson, J. C., \& Handler, S. M. (2011a). Nursing home deficiency citations for safety. J Aging Soc Policy, 23(1), 34-57. doi:10.1080/08959420.2011.532011.

Castle, N. (2011b). Nursing home deficiency citations for abuse. Journal of Applied Gerontology, 30(6), 719-743. doi: 10.1177/0733464811378262

Centers for Medicare and Medicaid Services (CMS). (2013). Nursing home data

Compendium 2013 Edition. Retrieved from http://www.cms.gov/Medicare/Provider-Enrollment-and Certification/CertificationandComplianc/downloads/nursinghomedatacompendiu m_508.Df 
Centers for Medicare and Medicaid Services (CMS). (2015). Nursing home data compendium 2015 Edition. Retrieved from https://www.cms.gov/Medicare/Provider-Enrollment-andCertification/CertificationandComplianc/Downloads/nursinghomedatacompendiu m_508-2015.pdf

Centers for Medicare and Medicaid Services (CMS). (2020). Five-star quality rating system. Calendar year provider standard surveys and deficiency files. Retrieved from: https://www.cms.gov/Medicare/Provider-Enrollment-andCertification/CertificationandComplianc/FSQRS

Centers for Disease Control and Prevention (CDC). (2017). Older adults falls. Important facts about falls. Retrieved from: https://www.cdc.gov/homeandrecreationalsafety/falls/adultfalls.html

Cheek, M., Nolan, D., \& Larsen-Orta, R. (2008). Rethinking the nursing home: Culture change makes headway in California. California Healthcare Foundation. Retrieved from: https://collections.nlm.nih.gov/master/borndig/101519310/RethinkingNursingHo meCulture.pdf

*Cohen, L. W., Zimmerman, S., Reed, D., Brown, P., Bowers, B. J., Nolet, K., . . Horn, S. (2016). The green house model of nursing home care in design and implementation. Health Services Research, 51 Suppl 1, 352-377. doi:10.1111/1475-6773.12418

Coleman, M.T., Looney, S., O’Brien, J., Ziegler C., Pastorino, C.A., \& Turner, C. (2002). The Eden Alternative: Findings after 1 year of implementation. Journal of Gerontology: Medical Sciences, 57A(7), M422-M427

Day, K., Carreon, D., \& Stump, C. (2000). The therapeutic design of environments for people with dementia: A review of the empirical research. The Gerontologist, 40(4), 397-416. doi:10.1093/geront/40.4.397

Department of Health and Human Services. (2019). Data brief: Trends in nursing home deficiencies. Retrieved from https://oig.hhs.gov/oas/reports/region9/91802010.pdf

Doty, M. M., Koren, M. J., \& Sturla, E. L. (2008). Culture change in nursing homes: How far have we come? Findings from the Commonweath Fund 2007 National Survey of Nursing Homes. Retrieved from http://www.commonwealthfund.org/Publications/FundReports/2008/May/Culture 
- Change-in-Nursing-Homes--How-Far-Have-We-Come Findings-From-TheCommonwealth-Fund-2007-Nati.aspx

Fritsch, T., Kwak, J., Grant, S., Lang, J., Montgomery, R.R., \& Basting, A.D. (2009). Impact of TimeSlips, a creative expression intervention program, on nursing home residents with dementia and their caregivers. The Gerontologist, 49(1), 117127. doi: 10.1093/geront/gnp008.

Gorina, Y., Schappert, S., Bercovitz, A., et al. (2014). Prevalence of incontinence among older Americans. National Center for Health Statistics. Vital Health Stat 3(36). Retrieved from: https://www.cdc.gov/nchs/data/series/sr_03/sr03_036.pdf

*Grabowski, D. C., Elliot, A., Leitzell, B., Cohen, L. W., \& Zimmerman, S. (2014a). Who are the innovators? Nursing homes implementing culture change. Gerontologist, 54 Suppl 1, S65-75. doi:10.1093/geront/gnt144

Grabowski, D.C., O’Malley, A.J., Afendulis, C.C., Caudry D.J., Elliot, A., \& Zimmerman, S. (2014b). Culture change and nursing home quality of care. The Gerontologist, 54(S1), S35-S45. doi: 10.1093/geront/gnt143

*Grabowski, D. C., Afendulis, C. C., Caudry, D. J., O'Malley, A. J., Kemper, P., \& Collaborative, T. R. (2016). The Impact of green house adoption on Medicare spending and utilization. Health Services Research, 51, 433-453. doi:10.1111/1475-6773.12438

Harris-Kojetin, L., Sengupta, M., Lendon, J.P., Rome, V., Valverde, R., \& Caffrey, C. (2019). Long-term care providers and services users in the United States, 20152016. National Center for Health Statistics. Vital Health Stat, 3(43). Retrieved from: https://www.cdc.gov/nchs/data/series/sr_03/sr03_43-508.pdf

Hermer, L., Bryant, N. S., Pucciarello, M., Mlynarczyk, C., \& Zhong, B. (2017). Does comprehensive culture change adoption via the household model enhance nursing home residents' psychosocial well-being? Innovation in Aging, 1(2), 1-13. doi:10.1093/geroni/igx033

Hermer, L., Cornelison, L., Kaup, M., L., Poey, J.,L., Drake, P., N., Stone, R., I., \& Doll, G., A. (2018). Person-centered care as facilitates by Kansas' PEAK 2.0 Medicaid pay-for-performance program and nursing home resident clinical outcomes. Innovation in Aging, 2(3), 1-12. doi: 10.1093/geroni/igy033

*Horn, S. D., Hudak, S. L., Barrett, R. S., Cohen, L. W., Reed, D. A., \& Zimmerman, S. 
(2016). Interpersonal care processes, falls, and hospitalizations in green house and other nursing homes. Seniors Housing \& Care Journal, 24(1), 31-46.

*Jenkens, R., Sult, T., Lessell, N., Hammer, D., \& Ortigara, A. (2011). Financial implications of the green house model. Seniors Housing \& Care Journal, 19(1), 3-22.

*Kane, R. A., Lum, T. Y., Cutler, L. J., Degenholtz, H. B., \& Tzy-Chyi, Y. (2007). Resident Outcomes in Small-House Nursing Homes: A Longitudinal Evaluation of the Initial Green House Program. Journal of the American Geriatrics Society, 55(6), 832-839. doi:10.1111/j.1532-5415.2007.01169.x

Konetzka, R.T., Brauner, D.J., Shega, J., \& Werner, R.M. (2014). The effects of public reporting on physical restraints and antipsychotic use in nursing home residents with severe cognitive impairment. Journal of the American Geriatrics Society, 62(3): 454-461. doi: 10.1111/jgs.12711

Koren, M. J. (2010). Person-centered care for nursing home residents: the culture-change movement. Health Affairs, 29(2). doi:10.1377/hlthaff.2009.0966

Kruschke, C. (2006). The Eden Alternative and Rosebud Nursing Center: does Eden Alternative improve resident outcomes in a long-term care setting? College of Education, Cardinal Stritch University.

Lepore, M. J., R. R. Shield, J. Looze, D. Tyler, V. Mor, \& S. C. Miller. (2015). Medicare and Medicaid reimbursement rages for nursing homes motivate select culture change practices but not comprehensive culture change. Journal of Aging and Social Policy, 27 (3): 215-31.

Li, J., \& Porock, D. (2014). Resident outcomes of person-centered care in long-term care: A narrative review of interventional research. International Journal of Nursing Studies, 51, 1395-1415. doi: http://dx.doi.org/10.1016/j.ijnurstu.2014.04.003

*Loe, M., \& Moore, C. D. (2012). From nursing home to green house: changing contexts of elder care in the united states. Journal of Applied Gerontology, 31(6), 755-763. doi:10.1177/0733464811401022

*Lum, T. Y., Kane, R. A., Cutler, L. J., \& Yu, T.-C. (2008). Effects of green house nursing homes on residents' families. Health Care Financing Rev, 30(2), 35-51

Miller, S. C., Looze, J., Shield, R., Clark, M. A., Lepore, M., Tyler, D., . . Mor, V. 
(2013). Culture change practice in U.S. nursing homes: Prevalence and variation by state Medicaid reimbursement policies. Gerontologist, 54(3), 434-445.

doi:10.1093/geront/gnt020

Miller, S.C., Mor, V., \& Burgess, J.J.F. (2016). Studying nursing home innovation: The green house model of nursing home care. Health Services Research, 51(1), 335343

Moher, D., Liberati, A., Tetzlaff, J., \& Altman, D. G. The PRISMA Group. (2009). Preferred reporting items for systematic reviews and meta-analyses: the PRISMA statement. PLoS Med, 6(7), 123-130.

Mukamel, D.B., Spector, W.D., Zinn, J.S., Huang, L., Weimer, D.L., \& Dozier, A. (2007). Nursing homes' response to the nursing home compare report card. Journal of Gerontology: Social Sciences, 62B (4), S218-S225. doi: 10.1093/geronb/62.4.s218

Needleman, J.S., Kim, N.K., Jung, H.Y., \& Unruh, M.A. (2018). Baseline rehospitalization rates of nursing homes in bundled payments for care improvement. Letters to the Editor/JAMDA, 19, 914-918. https://doi.org/10.1016/j.jamda.2018.05.022

Pioneer Network. (2011). Definitions of common terms used in long-term care and culture change. Retrieved from: https://www.pioneernetwork.net/wpcontent/uploads/2016/10/Definitions-of-Common-Terms-Used-in-Long-TermCare-and-Culture-Change.pdf

Rabig, J., Thomas, W., Kane, R. A., Cutler, L. J., \& McAlilly, S. (2006). Radical redesign of nursing homes: Applying the green house concept in Tupelo, Mississippi. Gerontologist, 46(4), 533-539. doi: 10.1093/geront/46.4.533

Ragsdale, V., \& McDougall, G. J., Jr. (2008). The changing face of long-term care: looking at the past decade. Issues Mental Health Nursing, 29(9), 992-1001. doi:10.1080/01612840802274818

Roberts, E., \& Pulay, A. (2018). Examining the nursing home physical environment through policy-driven culture change. Journal of Housing for the Elderly, 32(2), 241-262. doi:10.1080/02763893.2018.1431586 
Scott, W.R., \& Davis, G.F. (2007). Organizations and Organizing: Rational, Natural, and Open Systems Perspectives. Saddle River, New Jersey: Person Prentice Hall.

*Sharkey, S. S., Hudak, S., Horn, S. D., James, B., \& Howes, J. (2011). Frontline caregiver daily practices: A comparison study of traditional nursing homes and the green house project sites. Journal of the American Geriatrics Society, 59(1), 126-131. doi:10.1111/j.1532-5415.2010.03209.x

Shi, L. \& Singh, D.A. (2015). Long-Term Care. (6th ed.), Delivering Health Care in America: A systems Approach. Burlington, MA: Jones and Bartlett Learning.

Shier, V., Khodyakov, D., Cohen, L. W., Zimmerman, S., \& Saliba, D. (2014). What does the evidence really say about culture change in nursing homes? Gerontologist, 54 Suppl 1, S6-s16. doi:10.1093/geront/gnt147.

Shura, R., Siders, R.A., \& Dannefer, D. (2011). Culture change in long-term care: Participatory action research and the role of the resident. The Gerontologist, 51(2), 212-225. doi:10.1093/geront/gnq099.

Sterns, S., Miller, S. C., \& Allen, S. (2010). The complexity of implementing culture change practices in nursing homes. Journal of the American Medical Directors Association, 11(7), 511-518. doi:10.1016/j.jamda.2009.11.002

Stone, R. I., Reinhard, S.C., Bowers, B., ..., Jacobson, N. (2002). Evaluation of the Wellspring model for improving nursing home quality. Retrieved from: https://www.commonwealthfund.org/sites/default/files/documents/__media_files publications_fund_report_2002_aug_evaluation_of_the_wellspring_model_for_i mproving_nursing_home_quality_stone_wellspringevaluation_pdf.pdf

Temkin-Greener, H., Zheng, N.T., Cai, S., Zhao, H., \& Mukamel, D.B. (2010). Nursing home environment and organizational performance: Association with deficiency citations. Med Care, 48(4), 357-364. doi: 10.1097/MLR.0b013e3181ca3d70.

The Green House Project. (2020). Find a home. Retrieved from: https://www.thegreenhouseproject.org/about/find-a-home

Tyler, D. A., \& Lepore, M. J. (2017). Barriers and facilitators to adopting nursing home culture change. Journal of Clinical Outcomes Management, 24(11). 
U.S. Department of Health and Human Services. (2020). What is long-term care?

Washington, DC: HHS. 2013. Retrieved from: https://longtermcare.acl.gov/thebasics/what-is-long-term-care.html

Werner, R. M., Konetzka, R. T., \& Polsky, D. (2013). The effect of pay-for-performance in nursing homes: Evidence from state Medicaid programs. Health Services Research, 48, 1393-1414. doi:10.1111/1475-6773.12035

White-Chu, E.F., Graves, W.J., Godfrey, S.M., Bonner, A., \& Sloane, P. (2009). Beyond the medical model: the culture change revolution in long-term care. Journal of the American Medical Directors Association, 10, 370-378. doi: 10.1016/j.jamda.2009.04.004

*Wolff, M.L., Hassett, M., \& Kelly, A. (2016). An innovative quality assurance activity to reduce urinary tract infection rates in a green house skilled nursing setting. Annals of Long-Term Care: Clinical Care and Aging, 24(10), 17-20.

Wood, W., Harris, S., Snider, M., \& Patchel, S.A. (2005). Activity situations on an Alzheimer's disease special care unit and resident environmental interactions, time use, and affect. American Journal of Alzheimer's Disease and Other Dementias, 20(2), 105-118. doi:10.1177/153331750502000210

*Yoon, J. Y., Brown, R. L., Bowers, B. J., Sharkey, S. S., \& Horn, S. D. (2015). Longitudinal psychological outcomes of the small-scale nursing home model: a latent growth curve zero-inflated Poisson model. International Psychogeriatrics, 27(6), 1009-1016. doi:10.1017/s1041610214002865

*Yoon, J. Y., Brown, R. L., Bowers, B. J., Sharkey, S. S., \& Horn, S. D. (2016). The effects of the green house nursing home model on ADL function trajectory: A retrospective longitudinal study. International Journal of Nursing Studies, 53, 238-247. doi:10.1016/j.jinurstu.2015.07.010

Zimmerman, S., \& Cohen, L. W. (2010). Evidence behind the green house and similar models of nursing home care. Aging Health, 6(6), 717-737. doi:10.2217/ahe.10.66

Zimmerman, S., Bowers, B. J., Cohen, L. W., Grabowski, D. C., Horn, S. D., Kemper, P., $\&$ Collaborative, T. R. (2016). New Evidence on the green house model of nursing home care: Synthesis of findings and implications for policy, practice, and research. Health Services Research, 51, 475-496. doi:10.1111/14756773.12430 
CHAPTER 3: AIM 2

- Results

- Scenarios

- Scenario 1

- Scenario 2

- Scenario 3

- Scenario 4

- Scenario 5

- Scenario 6

CHAPTER 4: AIM 3

- Scenarios

- Scenario 1

- Scenario 2

- Scenario 3

- Scenario 4

- Scenario 5

- Scenario 6 
CHAPTER 3: AIM 2: RESULTS

\begin{tabular}{|c|c|c|c|c|}
\hline $\begin{array}{l}\text { Author(s), } \\
\text { Year }\end{array}$ & Sample Size & Study Design & GH Elements & Results/Outcomes \\
\hline $\begin{array}{l}\text { Afendulis et } \\
\text { al., } 2016\end{array}$ & $\begin{array}{l}15 \mathrm{GH} \text { homes and } 223 \\
\text { non-adopting NHs }\end{array}$ & $\begin{array}{l}\text { Quantitative design: Difference- } \\
\text { in-difference } \\
\text { A merge of the resident-level } \\
\text { minimum data set (MDS) } \\
\text { assessments with Medicare } \\
\text { inpatient claims for the period } \\
\text { 2005-2010 }\end{array}$ & $\begin{array}{l}\text { No GH elements } \\
\text { specified in the analysis }\end{array}$ & $\begin{array}{l}\text { Overall hospitalizations decreased in } \\
\text { GH homes (1.3 percent) comparing to } \\
\text { comparison NHs ( } 0.9 \text { percent) } \\
\text { A } 5.5 \text { percentage point decrease in all } \\
30 \text {-day rehospitalizations in GH homes } \\
\text { GH units experienced: } \\
15.8 \text { percent decrease in bedfast } \\
\text { residents } \\
45 \text { percent decrease in residents with } \\
\text { catheter } \\
38 \text { percent decrease in residents with } \\
\text { low-risk pressure ulcers } \\
\text { No impact on incontinence (low risk), } \\
\text { restraints, pressure ulcers (high-risk), } \\
\text { pain, and urinary tract infections }\end{array}$ \\
\hline $\begin{array}{l}\text { Bowers and } \\
\text { Nolet, } 2011\end{array}$ & $\begin{array}{l}68 \text { Shahbazim } \\
29 \text { licensed nursing staff } \\
8 \text { directors/assistant } \\
\text { directors of nursing } \\
11 \text { guides }\end{array}$ & $\begin{array}{l}\text { Qualitative Method } \\
\text { Grounded dimensional analysis }\end{array}$ & Staff & $\begin{array}{l}\text { Shahbazim: content in their new role \& } \\
\text { acquired a sense of empowerment. } \\
\text { Other Shahbazim: neutral, no } \\
\text { difference between GH homes and } \\
\text { traditional NHs } \\
\text { No supervision: sign of respect from } \\
\text { nurses } \\
\text { Issues with other staff and teamwork }\end{array}$ \\
\hline $\begin{array}{l}\text { Bowers and } \\
\text { Nolet, } 2014\end{array}$ & $\begin{array}{l}37 \text { nurses } \\
68 \text { CNAs } \\
11 \text { guides }\end{array}$ & $\begin{array}{l}\text { Qualitative Method } \\
\text { Dimensional analysis, a } \\
\text { "second generation" grounded } \\
\text { theory }\end{array}$ & Staff & $\begin{array}{l}\text { Integrated and parallel nursing models: } \\
\text { effective in communication and } \\
\text { teamwork. } \\
\text { Nurses experienced problems with } \\
\text { balancing their work. }\end{array}$ \\
\hline
\end{tabular}




\begin{tabular}{|l|l|l|l|l|}
\hline $\begin{array}{l}\text { Bowers et al., } \\
\text { 2016a }\end{array}$ & $\begin{array}{l}\text { Qualitative grounded theory } \\
\text { method }\end{array}$ & Staff & $\begin{array}{l}\text { Problem-solving: main solution to } \\
\text { maintain the GH model } \\
\text { Factors that strengthened or weakened } \\
\text { the implementation of the GH model: } \\
\text { Shahbazim: opportunities and } \\
\text { ability to solve problems } \\
\text { Leadership and regulatory support } \\
\text { Problems with financial plan } \\
\text { Competition for staff }\end{array}$ \\
\hline $\begin{array}{l}\text { Bowers et al., } \\
2016 \mathrm{~b}\end{array}$ & $\begin{array}{l}\text { 25 Shahbazim, 18 RNs } \\
\text { and LPNs,22 department } \\
\text { directors } \\
3 \text { individuals for QI } \\
3 \text { administrators } \\
6 \text { DONs, 5 physicians, and } \\
2 \text { NPs }\end{array}$ & $\begin{array}{l}\text { Qualitative grounded theory } \\
\text { method }\end{array}$ & $\begin{array}{l}\text { Staff } \\
\text { Physical structure }\end{array}$ & $\begin{array}{l}\text { GH homes with a low hospital transfer } \\
\text { rate leveraged resources to use for } \\
\text { care processes } \\
\text { GH homes with a high hospital } \\
\text { transfer rate did not take advantage of } \\
\text { the available resources to use for care } \\
\text { processes }\end{array}$ \\
\hline $\begin{array}{l}\text { Brown et al., } \\
2016\end{array}$ & $\begin{array}{l}\text { 26 GH units from 13 } \\
\text { organization } \\
15 \text { comparison units from } \\
8 \text { comparison NHs }\end{array}$ & $\begin{array}{l}\text { Observational study } \\
\text { Primary data from the GH } \\
\text { homes and comparison NHs } \\
\text { Secondary data from human } \\
\text { resources records on workforce } \\
\text { characteristics, turnover, and } \\
\text { staffing from 01-01-2011 to 06- } \\
30-2012\end{array}$ & $\begin{array}{l}\text { Shahbazim were found to be older } \\
\text { than CNAs in comparison NHs (mean } \\
\text { age 38.8 vs. 37.2) } \\
\text { No significant difference was found } \\
\text { related salary in the two settings. } \\
\text { GH homes experienced a low turnover } \\
\text { rate for Shahbazim and LPNs. }\end{array}$ \\
\hline
\end{tabular}




\begin{tabular}{|c|c|c|c|c|c|}
\hline $\begin{array}{l}\text { Cohen et al., } \\
2016\end{array}$ & \multicolumn{2}{|c|}{$\begin{array}{l}\text { Structured interviews: } \\
\text { staff from } 12 \mathrm{GH} \\
\text { organizations } \\
\text { Semi-structured } \\
\text { interviews: staff from } 9 \\
\text { GH organizations }\end{array}$} & $\begin{array}{l}\text { Mixed Methods, cross-sectional } \\
\text { study } \\
\text { Primary quantitative and } \\
\text { qualitative data } \\
\text { Secondary quantitative data } \\
\text { from 02-2012 to } 09-2014 \\
\text { Minimum dataset (MDS) } 3.0 \\
\text { and quality indicators retrieved } \\
\text { from Nursing Home Compare }\end{array}$ & $\begin{array}{l}\text { Staff: Empowerment } \\
\text { Physical structure } \\
\text { - Real home: natural } \\
\text { surroundings, } \\
\text { elements of real } \\
\quad \text { home, and home for } \\
\text { life } \\
\text { Elder-centered care } \\
\text { - Meaningful life: } \\
\text { autonomy and control } \\
\text { and purposeful living }\end{array}$ & $\begin{array}{l}\text { GH homes and legacy homes failed to } \\
\text { deliver full autonomy and control to } \\
\text { their residents. } \\
\text { GH homes were aligned with the GH } \\
\text { model design in terms of physical } \\
\text { structure and staff empowerment. } \\
\text { No significant difference between GH } \\
\text { homes and traditional NHs } \\
\text { concerning: } \\
\text { - Wound management } \\
\text { - Fall prevention } \\
\text { - Hospital transfers }\end{array}$ \\
\hline $\begin{array}{l}\text { Grabowski et } \\
\text { al., 2014a }\end{array}$ & $\begin{array}{l}291 \mathrm{NHs} \text { that } \\
\text { implemented } \\
\text { culture change } \\
11 \mathrm{GH} \text { homes } \\
16,740 \\
\text { traditional NHs }\end{array}$ & \multicolumn{2}{|c|}{$\begin{array}{l}\text { Quantitative design with logistic } \\
\text { regression } \\
\text { A combination of various data sources } \\
\text { Online Survey Certification and Reporting } \\
\text { (OSCAR) } \\
\text { CMS skilled nursing facility cost reports } \\
\text { State policy information } \\
\text { List from the Pioneer Network Board of } \\
\text { NHs that adopted culture change (2004- } \\
\text { 2011) }\end{array}$} & $\begin{array}{l}\text { No GH elements } \\
\text { specified in the analysis }\end{array}$ & $\begin{array}{l}\text { Status of culture change NH } \\
\text { implementers: } \\
\text { - Nonprofit } \\
\text { - Large size } \\
\text { - Less Medicaid and Medicare } \\
\text { residents } \\
\text { - At baseline, less health-related } \\
\text { deficiency citations } \\
\text { - At baseline, high number of LPNs } \\
\text { and CNAs } \\
\text { Increase in culture change adoption } \\
\text { was observed when states offered a } \\
\text { high Medicaid per diem }\end{array}$ \\
\hline
\end{tabular}




\begin{tabular}{|c|c|c|c|c|}
\hline $\begin{array}{l}\text { Grabowski et } \\
\text { al., } 2016\end{array}$ & $\begin{array}{l}15 \mathrm{GH} \text { homes } \\
\text { and } 223 \\
\text { nonadopting } \\
\mathrm{NHs}\end{array}$ & $\begin{array}{l}\text { Quantitative design with difference-in- } \\
\text { difference } \\
\text { A combination of Medicare claims, } \\
\text { enrollment data, and MDS from } 2005 \text { to } \\
2010\end{array}$ & $\begin{array}{l}\text { No GH elements } \\
\text { specified in the analysis }\end{array}$ & $\begin{array}{l}\text { The GH model had an influence on } \\
\text { Medicare Part A (plus hospice) } \\
\text { spending and utilization for GH home } \\
\text { compared to their legacy NHs. } \\
\text { A decrease of } \$ 7,746 \text { in annual } \\
\text { Medicare Part A (plus hospice) } \\
\text { spending was observed for GH homes. } \\
\text { The GH model did not have an impact } \\
\text { on Medicare Part A (plus hospice) } \\
\text { spending and utilization for the GH } \\
\text { organization (a combination of GH } \\
\text { home and legacy NH). }\end{array}$ \\
\hline $\begin{array}{l}\text { Horn et al., } \\
2016\end{array}$ & $\begin{array}{l}11 \mathrm{GH} \\
\text { organizations } \\
8 \text { comparison } \\
\mathrm{NHs}\end{array}$ & $\begin{array}{l}\text { Prospective, longitudinal, observational } \\
\text { study }\end{array}$ & $\begin{array}{l}\text { Staff } \\
\text { Physical structure }\end{array}$ & $\begin{array}{l}\text { A significant association between a } \\
\text { decrease in transfers and: } \\
\text { - The utilization of bladder scanners } \\
\text { and electrocardiogram services, } \\
\text { - Resident weights, and } \\
\text { - An increase in registered } \\
\text { nurse/licensed practical nurse } \\
\text { hours/resident day } \\
\text { Correlation between a decrease in falls } \\
\text { and: } \\
\text { - An increase in certified nursing } \\
\text { assistant hours/per resident day } \\
\text { (more pronounced in GH homes) } \\
\text { Association between a decrease in } \\
\text { falls and hospital transfers, and: } \\
\text { - An effective communication } \\
\text { between dietary staff and CNAs } \\
\text { related to the decrease observed in } \\
\text { their resident's weight. }\end{array}$ \\
\hline
\end{tabular}




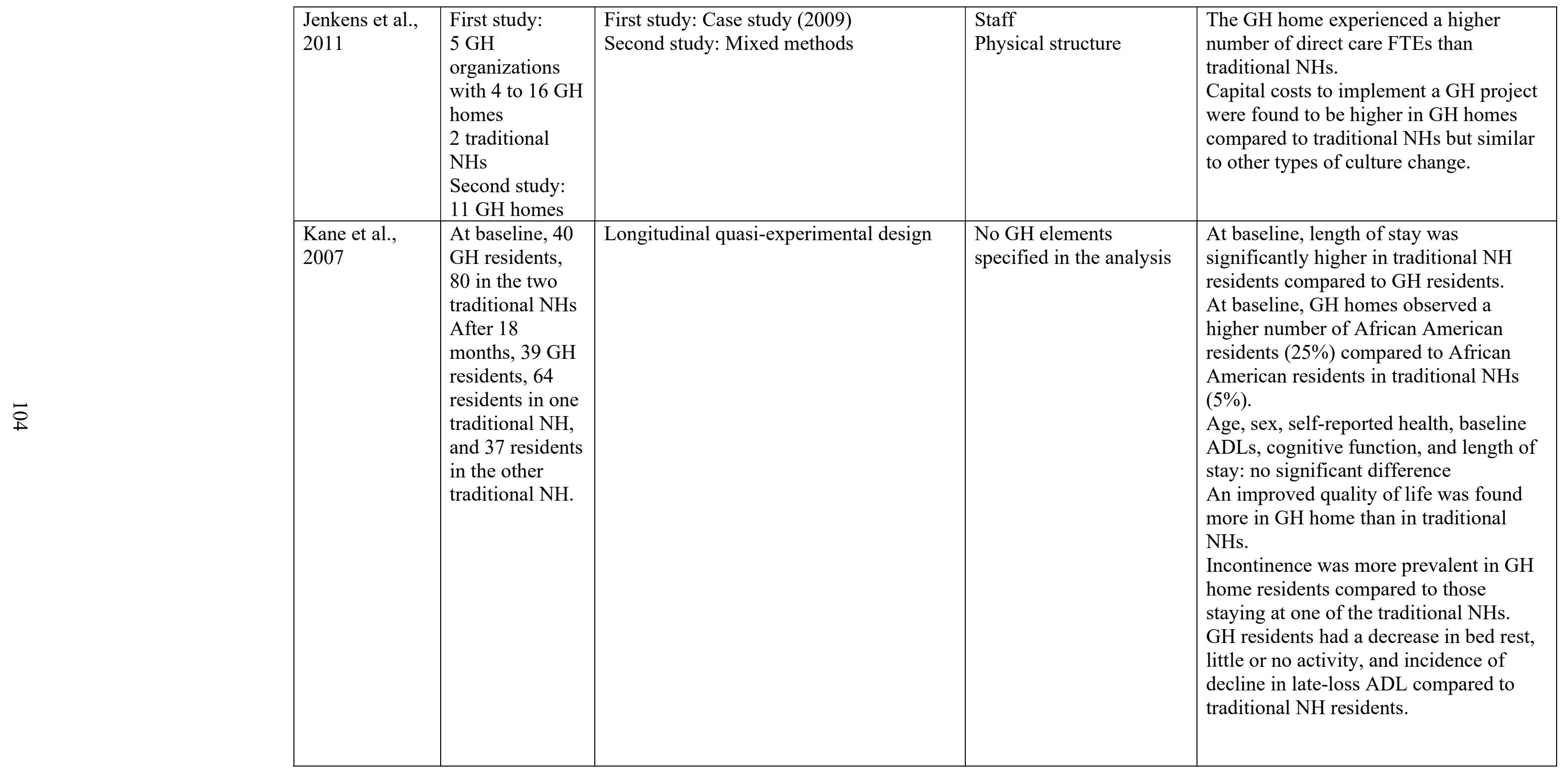




\begin{tabular}{|c|c|c|c|c|}
\hline $\begin{array}{l}\text { Lum et al., } \\
2008\end{array}$ & $\begin{array}{l}\text { At baseline, } 40 \\
\text { GH residents, } \\
80 \text { in the two } \\
\text { traditional NHs } \\
\text { After } 18 \\
\text { months, } 39 \mathrm{GH} \\
\text { residents, } 64 \\
\text { residents in one } \\
\text { traditional NH, } \\
\text { and } 37 \text { residents } \\
\text { in the other } \\
\text { traditional } \mathrm{NH} .\end{array}$ & Longitudinal quasi-experimental study & Family Engagement & $\begin{array}{l}\text { Family members: } \\
\text { - Not involved as much in laundry } \\
\text { and shopping in GH homes } \\
\text { compared to traditional NHs } \\
\text { - High satisfaction with GH homes } \\
\text { than traditional NHs, results, not } \\
\text { statistically significant }\end{array}$ \\
\hline $\begin{array}{l}\text { Sharkey et al., } \\
2011\end{array}$ & $\begin{array}{l}7 \mathrm{GH} \\
\text { organizations } \\
6 \text { control NHs }\end{array}$ & Observational study & Staff & $\begin{array}{l}\text { A high number of total nursing } \\
\text { HPRDs (RN, LPN, and CNA) (5.3) in } \\
\text { GH homes than in traditional } \\
\text { SNF units ( } 3.6 \text { ) } \\
\text { Shahbazim had } 1.56 \text { more total } \\
\text { nursing hours compared to CNAs in } \\
\text { traditional NHs } \\
\text { GH homes had } 2 \text { hours less in non- } \\
\text { nursing total hours than traditional } \\
\text { NHs. }\end{array}$ \\
\hline $\begin{array}{l}\text { Wolff et al., } \\
2016\end{array}$ & $\begin{array}{l}16 \text { cottage, } 192- \\
\text { bed skilled } \\
\text { nursing GH } \\
\text { home }\end{array}$ & $\begin{array}{l}\text { Qualitative Method } \\
\text { Quantitative Method }\end{array}$ & $\begin{array}{l}\text { No GH elements } \\
\text { specified in the analysis }\end{array}$ & $\begin{array}{l}\text { The incidence rate for UTIs per } 1000 \\
\text { bed-days decreased from } 2.25(2011) \\
\text { to } 1.08(2012) \text {. } \\
\text { With the introduction of the McGeer } \\
\text { criteria, the rate for UTIs per } 1000 \\
\text { bed-days was reduced from } 0.289 \\
\text { (2013) to } 0.145 \text { (2014). }\end{array}$ \\
\hline
\end{tabular}




\begin{tabular}{|c|c|c|c|c|}
\hline $\begin{array}{l}\text { Yoon et al., } \\
2015\end{array}$ & $\begin{array}{l}93 \mathrm{GH} \text { residents } \\
149 \text { traditional } \\
\text { NH residents }\end{array}$ & Quantitative method & Engagement & $\begin{array}{l}\text { Residents in GH home experienced a } \\
\text { high rate in psychological outcomes } \\
\text { (depression) than residents in } \\
\text { traditional NHs. } \\
\text { GH residents had an increase of the } \\
\text { likelihood of "not being socially } \\
\text { engaged" at a slower rate compared to } \\
\text { residents in traditional NHs. }\end{array}$ \\
\hline $\begin{array}{l}\text { Yoon et al., } \\
2016\end{array}$ & $\begin{array}{l}93 \mathrm{GH} \text { residents } \\
149 \text { traditional } \\
\text { NH residents }\end{array}$ & Retrospective longitudinal study & $\begin{array}{l}\text { No GH elements } \\
\text { specified in the analysis }\end{array}$ & $\begin{array}{l}\text { The mean ADL decreased during the } \\
\text { period of the study. } \\
\text { The difference in the rate of change in } \\
\text { ADL for GH residents to traditional } \\
\text { NH residents was not statistically } \\
\text { significant }(\beta=\beta-0.09, p=.637) \text {. }\end{array}$ \\
\hline
\end{tabular}




\section{CHAPTER 3: AIM 2}

\section{Scenarios}

\section{Scenario 1}

In this scenario, variables with LNE values were removed. Those variables were age, sex, and race. Observations fluctuated across the years and there was not an exact pattern to report. In total, there were 1,208 observations with $55 \mathrm{GH}$ facilities and 1,153 non-GH facilities across the 18 years. The table below describes the number of GH facilities and the number of non-GH facilities per year.

\begin{tabular}{|l|l|l|l|}
\hline Year & GH Facilities & Non-GH Facilities & Total \\
\hline 2000 & 0 & 62 & 62 \\
\hline 2001 & 0 & 61 & 61 \\
\hline 2002 & 0 & 59 & 59 \\
\hline 2003 & 0 & 62 & 62 \\
\hline 2004 & 1 & 65 & 66 \\
\hline 2005 & 1 & 58 & 59 \\
\hline 2006 & 1 & 53 & 54 \\
\hline 2007 & 4 & 51 & 55 \\
\hline 2008 & 3 & 56 & 59 \\
\hline 2009 & 4 & 54 & 58 \\
\hline 2010 & 4 & 54 & 58 \\
\hline 2011 & 4 & 88 & 92 \\
\hline 2012 & 4 & 72 & 76 \\
\hline 2013 & 7 & 83 & 90 \\
\hline 2014 & 8 & 73 & 81 \\
\hline 2015 & 4 & 68 & 72 \\
\hline 2016 & 7 & 70 & 77 \\
\hline 2017 & 3 & 64 & 67 \\
\hline Total & 1,153 & 55 & 1,208 \\
\hline
\end{tabular}

Scenario 1

\section{Scenario 2}

Within this scenario, we replaced the variables with LNE values with a calculated value. To obtain this calculated value, we created an estimate for the number of residents first by multiplying the total of beds with the occupancy rate and second by dividing one 
over this number. Then, this estimate was used to replace the variables with LNE values. The replaced variables were age, sex, and race. Observations did not change with 5,040 observations in total with $175 \mathrm{GH}$ facilities and 4,865 non-GH facilities across 18 years.

\section{Scenario 3}

In this case, we interpolated the variables with LNE values. To create the interpolated variables, the variables of interest were treated as the outcome variables and the year variable was treated as the independent variable. Then, the interpolated variables were used to replace the variables with LNE values. The replaced variables were age, sex, and race. A change in the number of total of observations did not change with 5,040 observations in total with $175 \mathrm{GH}$ facilities and 4,865 non-GH facilities across 18 years.

\section{Scenario 4}

In this scenario, we removed variables with LNE values and other missing values. Those variables were age, sex, and race. Observations fluctuated across the years and there was not an exact pattern to report. In total, there were 1,192 observations with 48 $\mathrm{GH}$ facilities and 1,144 non-GH facilities across the 18 years. This table below describes the number of GH facilities and the number of non-GH facilities per year.

\begin{tabular}{|l|l|l|l|}
\hline Year & GH Facilities & Non-GH Facilities & Total \\
\hline 2000 & 0 & 60 & 60 \\
\hline 2001 & 0 & 60 & 60 \\
\hline 2002 & 0 & 58 & 58 \\
\hline 2003 & 0 & 61 & 61 \\
\hline 2004 & 0 & 65 & 65 \\
\hline 2005 & 0 & 58 & 58 \\
\hline 2006 & 0 & 52 & 52 \\
\hline 2007 & 3 & 50 & 53 \\
\hline 2008 & 2 & 56 & 58 \\
\hline 2009 & 3 & 53 & 56 \\
\hline 2010 & 3 & 53 & 56 \\
\hline 2011 & 4 & 88 & 92 \\
\hline 2012 & 4 & 72 & 76 \\
\hline
\end{tabular}




\begin{tabular}{|l|l|l|l|}
\hline 2013 & 7 & 83 & 90 \\
\hline 2014 & 8 & 73 & 81 \\
\hline 2015 & 4 & 68 & 72 \\
\hline 2016 & 7 & 70 & 77 \\
\hline 2017 & 3 & 64 & 67 \\
\hline Total & 1,144 & 48 & 1,192 \\
\hline
\end{tabular}

Scenario 4

\section{Scenario 5}

Within this scenario, we replaced the variables with LNE values and other missing values with a calculated value called LNE_replace. To obtain the LNE_replace variable, we created an estimated value for the number of residents first by multiplying the total number of beds with the occupancy rate and second by dividing the number one over the LNE_replace variable. Then, this estimated value was used to replace the variables with LNE values. The replaced variables were age, sex, and race. Observations did not change with 5,040 observations in total with $175 \mathrm{GH}$ facilities and 4,865 non-GH facilities across 18 years.

\section{Scenario 6}

In this case, we interpolated the variables with LNE values and other missing values. To create the interpolated variables, the variables of interest were treated as the outcome variables and the year variable was treated as the independent variable. Then, the interpolated variables were used to replace the variables with LNE values. The replaced variables were age, sex, and race. A change in the number of total of observations did not change with 5,040 observations in total with $175 \mathrm{GH}$ facilities and 4,865 non-GH facilities across 18 years. 


\section{CHAPTER 4: AIM 3}

\section{Scenarios}

\section{Scenario 1}

In this scenario, variables with LNE values were removed. Those variables were the prevalence of falls, the prevalence of bladder incontinence, the prevalence of bowel incontinence, age, sex, and race. Observations fluctuated across the years and there was not an exact pattern to report. In total, there were 1,208 observations with $55 \mathrm{GH}$ facilities and 1,153 non-GH facilities across the 18 years. Table 1 describes the number of GH facilities and the number of non-GH facilities per year.

\begin{tabular}{|l|l|l|l|}
\hline Year & GH Facilities & Non-GH Facilities & Total \\
\hline 2000 & 0 & 62 & 62 \\
\hline 2001 & 0 & 61 & 61 \\
\hline 2002 & 0 & 59 & 59 \\
\hline 2003 & 0 & 62 & 62 \\
\hline 2004 & 1 & 65 & 66 \\
\hline 2005 & 1 & 58 & 59 \\
\hline 2006 & 1 & 53 & 54 \\
\hline 2007 & 4 & 51 & 55 \\
\hline 2008 & 3 & 56 & 59 \\
\hline 2009 & 4 & 54 & 58 \\
\hline 2010 & 4 & 54 & 58 \\
\hline 2011 & 4 & 88 & 92 \\
\hline 2012 & 4 & 72 & 76 \\
\hline 2013 & 7 & 83 & 90 \\
\hline 2014 & 8 & 73 & 81 \\
\hline 2015 & 4 & 68 & 72 \\
\hline 2016 & 7 & 70 & 77 \\
\hline 2017 & 3 & 64 & 67 \\
\hline Total & 1,153 & 55 & 1,208 \\
\hline Scar 1 & & & \\
\hline
\end{tabular}

Scenario 1

\section{Scenario 2}

Within this scenario, we replaced the variables with LNE values with a calculated value. To obtain this calculated value, we created an estimate for the number of residents first by multiplying the total of beds with the occupancy rate and second by dividing one 
over this number. Then, this estimate was used to replace the variables with LNE values. The replaced variables were the prevalence of falls, the prevalence of bladder incontinence, the prevalence of bowel incontinence, age, sex, and race. Observations did not change with 5,040 observations in total with $175 \mathrm{GH}$ facilities and 4,865 non-GH facilities across 18 years.

\section{Scenario 3}

In this case, we interpolated the variables with LNE values. To create the interpolated variables, the variables of interest were treated as the outcome variables and the year variable was treated as the independent variable. Then, the interpolated variables were used to replace the variables with LNE values. The replaced variables were the prevalence of falls, the prevalence of bladder incontinence, the prevalence of bowel incontinence, age, sex, and race. A change in the number of total of observations did not change with 5,040 observations in total with $175 \mathrm{GH}$ facilities and 4,865 non-GH facilities across 18 years.

\section{Scenario 4}

In this case, we removed variables with LNE values and other missing values. Those variables were the prevalence of falls, the prevalence of bladder incontinence, the prevalence of bowel incontinence, age, sex, and race. Observations fluctuated across the years and there was not an exact pattern to report. In total, there were 1,192 observations with $48 \mathrm{GH}$ facilities and 1,144 non-GH facilities across the 18 years. Table 2 describes the number of GH facilities and the number of non-GH facilities per year.

\begin{tabular}{|l|l|l|l|}
\hline Year & GH Facilities & Non-GH Facilities & Total \\
\hline 2000 & 0 & 60 & 60 \\
\hline 2001 & 0 & 60 & 60 \\
\hline 2002 & 0 & 58 & 58 \\
\hline
\end{tabular}




\begin{tabular}{|l|l|l|l|}
\hline 2003 & 0 & 61 & 61 \\
\hline 2004 & 0 & 65 & 65 \\
\hline 2005 & 0 & 58 & 58 \\
\hline 2006 & 0 & 52 & 52 \\
\hline 2007 & 3 & 50 & 53 \\
\hline 2008 & 2 & 56 & 58 \\
\hline 2009 & 3 & 53 & 56 \\
\hline 2010 & 3 & 53 & 56 \\
\hline 2011 & 4 & 88 & 92 \\
\hline 2012 & 4 & 72 & 76 \\
\hline 2013 & 7 & 83 & 90 \\
\hline 2014 & 8 & 73 & 81 \\
\hline 2015 & 4 & 68 & 72 \\
\hline 2016 & 7 & 70 & 77 \\
\hline 2017 & 3 & 64 & 67 \\
\hline Total & 1,144 & 48 & 1,192 \\
\hline
\end{tabular}

Scenario 4

\section{Scenario 5}

Within this scenario, we replaced the variables with LNE values and other missing values with a calculated value called LNE_replace. To obtain the LNE_replace variable, we created an estimated value for the number of residents first by multiplying the total number of beds with the occupancy rate and second by dividing the number one over the LNE_replace variable. Then, this estimated value was used to replace the variables with LNE values. The replaced variables were the prevalence of falls, the prevalence of bladder incontinence, the prevalence of bowel incontinence, age, sex, and race.

Observations did not change with 5,040 observations in total with $175 \mathrm{GH}$ facilities and 4,865 non-GH facilities across 18 years.

\section{Scenario 6}

In this case, we interpolated the variables with LNE values and other missing values. To create the interpolated variables, the variables of interest were treated as the outcome variables and the year variable was treated as the independent variable. Then, 
the interpolated variables were used to replace the variables with LNE values. The replaced variables were the prevalence of falls, the prevalence of bladder incontinence, the prevalence of bowel incontinence, age, sex, and race. A change in the number of total of observations did not change with 5,040 observations in total with $175 \mathrm{GH}$ facilities and 4,865 non-GH facilities across 18 years. 


\section{CURRICULUM VITA}

NAME: $\quad$ Deborah Kaminka Niyongabo

ADDRESS: Department of Health Management and Systems Sciences

School of Public Health and Information Sciences

University of Louisville

Louisville, Kentucky 40292

DOB: $\quad$ Brussels, Belgium - May 12, 1988

EDUCATION

\& TRAINING:

A.A., General Studies Montgomery College

2006-2010

B.S., Public Health Sciences

University of Maryland

2011-2012

M.P.H., Global Health

Liberty University

2013-2015

Ph.D., Health Management

University of Louisville

2016-2020

POSITIONS HELD

Graduate Research Assistant, Department of Health Management and Systems Sciences, School of Public Health and Information Sciences, University of Louisville

2016-2020

Project Title Mountain States Regional Genetics Network, University of Louisville

Project Title Law Enforcement Assisted Diversion (LEAD), University of Louisville 
Research Assistant, Division of Infectious Diseases, School of Medicine, University of Louisville

2016-2017

Public Health Intern, Samaritan's Purse

Niamey, Niger

2015

Youth Program Coordinator

African Immigrant and Refugee Foundation

Washington, D.C.

2012-2013

Intern

African Immigrant and Refugee Foundation

Washington, D.C.

2012

PUBLISHED PEER REVIEWED ARTICLES:

Carrico RM, Goss L, Wojcik J, Broughton-Miller K, Pentecost K, Frisbie M, Kotey S, Niyongabo D, Benns M, Raghuram A, Logsdon MC. (2017). Postsplenectomy vaccination guideline adherence: opportunities for improvement. Journal of the American Association of Nurse, 29(10): 612-617

PRESENTATIONS:

Poster presentations at two conferences

APHA, 2018 \& Academy Health, 2019

PROFESSIONAL SERVICE AFFILIATIONS:

Member

Academy Health

2017-present

Member and Reviewer

Academy of Management

2018-present 